\title{
Bounds for graph regularity and removal lemmas
}

\author{
David Conlon* Jacob Fox ${ }^{\dagger}$
}

\begin{abstract}
We show, for any positive integer $k$, that there exists a graph in which any equitable partition of its vertices into $k$ parts has at least $c k^{2} / \log ^{*} k$ pairs of parts which are not $\epsilon$-regular, where $c, \epsilon>0$ are absolute constants. This bound is tight up to the constant $c$ and addresses a question of Gowers on the number of irregular pairs in Szemerédi's regularity lemma.

In order to gain some control over irregular pairs, another regularity lemma, known as the strong regularity lemma, was developed by Alon, Fischer, Krivelevich, and Szegedy. For this lemma, we prove a lower bound of wowzer-type, which is one level higher in the Ackermann hierarchy than the tower function, on the number of parts in the strong regularity lemma, essentially matching the upper bound. On the other hand, for the induced graph removal lemma, the standard application of the strong regularity lemma, we find a different proof which yields a tower-type bound.

We also discuss bounds on several related regularity lemmas, including the weak regularity lemma of Frieze and Kannan and the recently established regular approximation theorem. In particular, we show that a weak partition with approximation parameter $\epsilon$ may require as many as $2^{\Omega\left(\epsilon^{-2}\right)}$ parts. This is tight up to the implied constant and solves a problem studied by Lovász and Szegedy.
\end{abstract}

\section{Introduction}

Originally developed by Szemerédi as part of his proof of the celebrated Erdős-Turán conjecture on long arithmetic progressions in dense subsets of the integers [39], Szemerédi's regularity lemma [40] has become a central tool in extremal combinatorics. Roughly speaking, the lemma says that the vertex set of any graph may be partitioned into a small number of parts such that the bipartite subgraph between almost every pair of parts behaves in a random-like fashion.

Given two subsets $X$ and $Y$ of a graph $G$, we write $d(X, Y)$ for the density of edges between $X$ and $Y$. The pair $(X, Y)$ is said to be $(\epsilon, \delta)$-regular if for some $\alpha$ and all $X^{\prime} \subset X$ and $Y^{\prime} \subset Y$ with $\left|X^{\prime}\right| \geq \delta|X|$ and $\left|Y^{\prime}\right| \geq \delta|Y|$, we have $\alpha<d\left(X^{\prime}, Y^{\prime}\right)<\alpha+\epsilon$. In the case where $\delta=\epsilon$, we say that the pair $(X, Y)$ is $\epsilon$-regular. By saying that a pair of parts is random-like, we mean that they are $(\epsilon, \delta)$-regular with $\epsilon$ and $\delta$ small, a property which is easily seen to be satisfied with high probability by a random bipartite graph. We will also ask that the different parts be of comparable size, that is, that the partition $V(G)=V_{1} \cup \ldots \cup V_{k}$ be equitable, that is, ||$V_{i}|-| V_{j}|| \leq 1$ for all $i$ and $j$.

\footnotetext{
*St John's College, Cambridge CB2 1TP, United Kingdom. E-mail: d.conlon@dpmms.cam.ac.uk. Research supported by a Royal Society University Research Fellowship.

${ }^{\dagger}$ Department of Mathematics, MIT, Cambridge, MA 02139-4307. E-mail: fox@math.mit.edu. Research supported by a Simons Fellowship and NSF grant DMS-1069197.
} 
The regularity lemma now states that for each $\epsilon, \delta, \eta>0$, there is a positive integer $M=M(\epsilon, \delta, \eta)$ such that the vertices of any graph $G$ can be equitably partitioned $V(G)=V_{1} \cup \ldots \cup V_{M}$ into $M$ parts where all but at most an $\eta$ fraction of the pairs $\left(V_{i}, V_{j}\right)$ are $(\epsilon, \delta)$-regular. We shall say that such a partition is $(\epsilon, \delta, \eta)$-regular and simply $\epsilon$-regular in the case $\epsilon=\delta=\eta$. For more background on the regularity lemma, see the excellent surveys by Komlós and Simonovits [27] and Rödl and Schacht [33].

Use of the regularity lemma is now widespread throughout graph theory. However, one of the earliest applications, the triangle removal lemma of Ruzsa and Szemerédi [36], remains the standard example. It states that for any $\epsilon>0$ there exists $\delta>0$ such that any graph on $n$ vertices with at most $\delta n^{3}$ triangles can be made triangle-free by removing $\epsilon n^{2}$ edges. It easily implies Roth's theorem [34] on 3term arithmetic progressions in dense sets of integers, and Solymosi [37] showed that it further implies the stronger corners theorem of Ajtai and Szemerédi [1, which states that any dense subset of the integer grid contains the vertices of an axis-aligned isosceles triangle. This result was extended to all graphs in [17, 3]. The extension, known as the graph removal lemma, says that given a graph $H$ on $h$ vertices and $\epsilon>0$ there exists $\delta>0$ such that any graph on $n$ vertices with at most $\delta n^{h}$ copies of $H$ can be made $H$-free by removing $\epsilon n^{2}$ edges.

One disadvantage of applying the regularity lemma to prove this theorem is the bounds that it gives for the size of $\delta$ in terms of $\epsilon$. The proof of the regularity lemma yields a bound of tower-type for the number of pieces in the partition. When this is applied to graph removal, it gives a bound for $\delta^{-1}$ which is a tower of twos of height polynomial in $\epsilon^{-1}$. Surprisingly, any hope that a better bound for the regularity lemma might be found was put to rest by Gowers [22], who showed that there are graphs for which a tower-type number of parts are required in order to obtain a regular partition.

To be more precise, the proof of the regularity lemma shows that $M(\epsilon, \delta, \eta)$ can be taken to be a tower of twos of height proportional to $\epsilon^{-2} \delta^{-2} \eta^{-1}$. Gowers' result, described in [13] as a tour de force, is a lower bound, with $c=1 / 16$, on $M\left(1-\delta^{c}, \delta, 1-\delta^{c}\right)$ which is a tower of twos of height proportional to $\delta^{-c}$. As Gowers notes, it is an easy exercise to translate lower bounds for small $\delta$ and large $\epsilon$ into lower bounds for large $\delta$ and small $\epsilon$ which are also of tower-type. However, the natural question, discussed by Szemerédi [40, Komlós and Simonovits [27], and Gowers [22], of determining the dependency of $M(\epsilon, \delta, \eta)$ on $\eta$, which measures the fraction of allowed irregular pairs, has remained open. This is the first problem we will address here, showing that the dependence is again of tower-type.

This does not mean that better bounds cannot be proved for the graph removal lemma. Recently, an alternative proof was found by the second author [18], allowing one to show that $\delta^{-1}$ may be taken to be a tower of twos of height $O\left(\log \epsilon^{-1}\right)$, better than one could possibly do using regularity. Though this remains quite far from the lower bound of $\epsilon^{-O\left(\log \epsilon^{-1}\right)}$, it clears a significant hurdle.

The second major theme of this paper is a proof of the induced graph removal lemma which similarly bypasses a natural obstacle. Let $H$ be a graph on $h$ vertices. The induced graph removal lemma, proved by Alon, Fischer, Krivelevich, and Szegedy [5], states that for any $\epsilon>0$ there exists $\delta>0$ such that any graph on $n$ vertices with at most $\delta n^{h}$ induced copies of $H$ may be made induced $H$-free by adding or deleting at most $\epsilon n^{2}$ edges.

This result, which easily implies the graph removal lemma, does not readily follow from the same 
technique used to prove the graph removal lemma, mainly because of the possibility of irregular pairs in the regularity partition. To overcome this issue, Alon, Fischer, Krivelevich, and Szegedy [5] developed a strenghthening of Szemerédi's regularity lemma. Roughly, it gives an equitable partition $\mathcal{A}$ and an equitable refinement $\mathcal{B}$ of $\mathcal{A}$ such that $\mathcal{A}$ and $\mathcal{B}$ are both regular, with the guaranteed regularity of $\mathcal{B}$ allowed to depend on the size of $\mathcal{A}$, and the edge density between almost all pairs of parts in $\mathcal{B}$ close to the edge density between the pair of parts in $\mathcal{A}$ that they lie in.

The proof of the strong regularity lemma involves iterative applications of Szemerédi's regularity lemma. This causes the upper bound on the number of parts in $\mathcal{B}$ to grow as a wowzer function, which is one level higher in the Ackermann hierarchy than the tower function. In order to get an improved bound for its various applications, one may hope that an improved bound of tower-type could be established. We show that no such bound exists. In fact, we will show that a seemingly weaker statement requires wowzer-type bounds. On the other hand, we give an alternative proof of the induced graph removal lemma, allowing one to show that $\delta^{-1}$ may be taken to be a tower of twos of height polynomial in $\epsilon^{-1}$, better than one could possibly achieve using the strong regularity lemma. We also make progress on determining bounds for various related regularity lemmas, including the Frieze-Kannan weak regularity lemma [19, 20] and the regular approximation theorem, due independently to Lovász and Szegedy [29] and to Rödl and Schacht [32]. We discuss all these contributions in more detail in the sections below.

\subsection{The number of irregular pairs}

The role of $\eta$ in the regularity lemma is to measure how many pairs of subsets in the partition are regular. If a partition into $k$ pieces is $(\epsilon, \delta, \eta)$-regular, then there will be at most $\eta\left(\begin{array}{l}k \\ 2\end{array}\right)$ irregular pairs in the partition. Szemerédi [40] wrote that it would be interesting to determine if the assertion of the regularity lemma holds when we do not allow any irregular pairs. This question remained unanswered for a long time until it was observed by Lovász, Seymour, Trotter, and Alon, Duke, Lefmann, Rödl, and Yuster [3] that irregular pairs can be necessary. The simple example of the half-graph shows that this is indeed the case. The half-graph is a bipartite graph with vertex sets $A=\left\{a_{1}, \ldots, a_{n}\right\}$ and $B=\left\{b_{1}, \ldots, b_{n}\right\}$ in which $\left(a_{i}, b_{j}\right)$ is an edge if and only if $i \leq j$. Any partition of this graph into $M$ parts will have $\Omega(M)$ irregular pairs. In other words, $M(\epsilon, \delta, \eta)$ must grow at least linearly in $\eta^{-1}$.

However, the number of irregular pairs, or, in other words, the dependence of $M(\epsilon, \delta, \eta)$ on $\eta^{-1}$ with $\epsilon$ and $\delta$ fixed, has not been well understood despite being asked several times, including by Komlós and Simonovits [27] and, more explicitly, by Gowers [22]. This problem and related problems have continued to attract interest (see, e.g., [26], [30]). The linear bound obtained from the half-graph appears to be the only bound in the literature for this problem.

For fixed constants $\epsilon$ and $\delta$ and each $M$, we give a construction in which any partition into $M$ parts has at least $c M^{2} / \log ^{*} M$ irregular parts, where $c>0$ is an absolute constant, and this is tight apart from the constant $c$. The iterated $\operatorname{logarithm} \log ^{*} n$ is the number of times the logarithm function needs to be applied to get a number which is at most 1 . That is, $\log ^{*} x=0$ if $x \leq 1$ and otherwise $\log ^{*} x=1+\log ^{*}(\log x)$ denotes the iterated logarithm. In other words, the dependence on $\eta$ in 
$M(\epsilon, \delta, \eta)$ is indeed a tower of twos of height proportional to $\Theta\left(\eta^{-1}\right)$.

Theorem 1.1 There are absolute constants $c, \epsilon, \delta>0$ such that for every $k$ there is a graph in which every equitable partition of the graph into $k$ parts has at least $c k^{2} / \log ^{*} k$ pairs of parts which are not $(\epsilon, \delta)$-regular. In other words, $M(\epsilon, \delta, \eta)$ is at least a tower of twos of height $\mathrm{c}^{-1}$.

We prove Theorem 1.1 with $\epsilon=\frac{1}{2}, \delta=2^{-500}$, and $c=2^{-700}$, and we make no attempt to optimize constants. The proof of Theorem 1.1 can be easily modified to obtain the same result with $\epsilon$ tending to 1 at the expense of having $\delta$ and $c$ tending to 0 .

In the important special case where $\epsilon=\delta=\eta$, we let $M(\epsilon)=M(\epsilon, \delta, \eta)$. Gowers 22] gave two different constructions giving lower bounds on $M(\epsilon)$. The first construction is simpler, but the lower bound it gives is a tower of twos of height only logarithmic in $\epsilon^{-1}$. The second construction gives a lower bound which is a tower of twos of height $\epsilon^{-1 / 16}$, but is more complicated. Theorem 1.1 also gives a lower bound on $M(\epsilon)$ which is a tower of twos of height polynomial in $\epsilon^{-1}$, in fact linear in $\epsilon^{-1}$, and the construction is a bit simpler. Unfortunately, the proof that it works, which builds upon Gowers' simpler first proof, is still rather complicated and delicate.

We give a rough idea of how the graph $G$ used to prove Theorem 1.1 is constructed. The graph $G$ has a sequence of vertex partitions $P_{1}, \ldots, P_{s}$, with $P_{i+1}$ a refinement of $P_{i}$ for $1 \leq i \leq s-1$, and the number of parts of $P_{i+1}$ is roughly exponential in the number of parts of $P_{i}$. For each $i, 1 \leq i \leq s-1$, we pick a random graph $G_{i}$ with vertex set $P_{i}$, where each edge is picked independently with probability $p_{i}$. For every two vertex subsets $X, Y \in P_{i}$ of $G$ which are adjacent in $G_{i}$, we take random vertex partitions $X=X_{Y}^{1} \cup X_{Y}^{2}$ and $Y=Y_{X}^{1} \cup Y_{X}^{2}$ into parts of equal size, with each of these parts the union of parts of $P_{i+1}$. Then, for $d=1,2$, we add the edges to $G$ between $X_{Y}^{d}$ and $Y_{X}^{d}$. We will show that with positive probability the graph $G$ constructed above has the desired properties for Theorem 1.1. In fact, in Theorem 3.1, we will show that it has the stronger property that any $(\epsilon, \delta, \eta)$-regular vertex partition of $G$ is close to being a refinement of $P_{s}$.

A novelty of our construction, not present in the constructions of Gowers, is the use of the random graphs $G_{i}$, which allow us to control the number of irregular pairs. Instead, for every pair of parts $X, Y$ in $P_{i}$, Gowers [22] introduces or deletes some edges between them so as to make the pair of parts far from regular. To prove the desired result, we first establish several lemmas on the edge distribution in $G$. The construction is general enough and Theorem 3.1 strong enough that we also use it to establish a wowzer-type lower bound for the strong regularity lemma, as described in the next subsection.

\subsection{The strong regularity lemma}

Before stating the strong regularity lemma, we next define a notion of closeness between an equitable partition and an equitable refinement of this partition. For an equitable partition $\mathcal{A}=\left\{V_{i} \mid 1 \leq i \leq k\right\}$ of $V(G)$ and an equitable refinement $\mathcal{B}=\left\{V_{i, j} \mid 1 \leq i \leq k, 1 \leq j \leq \ell\right\}$ of $\mathcal{A}$, we say that $\mathcal{B}$ is $\epsilon$-close to $\mathcal{A}$ if the following is satisfied. All $1 \leq i \leq i^{\prime} \leq k$ but at most $\epsilon k^{2}$ of them are such that for all $1 \leq j, j^{\prime} \leq \ell$ but at most $\epsilon \ell^{2}$ of them $\left|d\left(V_{i}, V_{i^{\prime}}\right)-d\left(V_{i, j}, V_{i^{\prime}, j^{\prime}}\right)\right|<\epsilon$ holds. This notion roughly says 
that $\mathcal{B}$ is an approximation of $\mathcal{A}$. We are now ready to state the strong regularity lemma of Alon, Fischer, Krivelevich, and Szegedy [5].

Lemma 1.1 (Strong regularity lemma) For every function $f: \mathbb{N} \rightarrow(0,1)$ there exists a number $S=S(f)$ with the following property. For every graph $G=(V, E)$, there is an equitable partition $\mathcal{A}$ of the vertex set $V$ and an equitable refinement $\mathcal{B}$ of $\mathcal{A}$ with $|\mathcal{B}| \leq S$ such that the partition $\mathcal{A}$ is $f(1)$-regular, the partition $\mathcal{B}$ is $f(|\mathcal{A}|)$-regular, and $\mathcal{B}$ is $f(1)$-close to $\mathcal{A}$.

The upper bound on $S$, the number of parts of $\mathcal{B}$, that the proof gives is of wowzer-type, which is one level higher in the Ackermann hierarchy than the tower function. The tower function is defined by $T(1)=2$, and $T(n)=2^{T(n-1)}$ for $n \geq 2$. The wowzer function $W(n)$ is defined by $W(1)=2$ and $W(n)=T(W(n-1))$. For reasonable choices of the function $f$, which is the case for all known applications, such as those for which $1 / f$ is an increasing function which is at least a constant number of iterations of the logarithm function, the upper bound on $S(f)$ is at least wowzer in a power of $\epsilon=f(1)$. Recall that $M(\epsilon)$, the number of parts required for Szemerédi's regularity lemma, grows as a tower of height a power of $\epsilon^{-1}$. The precise upper bound on the number of parts in the strong regularity lemma is defined as follows. Let $W_{1}=M(\epsilon)$ and $W_{i+1}=M\left(2 f\left(W_{i}\right) / W_{i}^{2}\right)$. The proof of the strong regularity lemma [5] shows that $S(f)=512 \epsilon^{-4} W_{j}$ with $j=64 \epsilon^{-4}$ satisfies the required property.

For a partition $\mathcal{P}: V(G)=V_{1} \cup \ldots \cup V_{k}$ of the vertex set of a graph $G$, the mean square density of $\mathcal{P}$ is defined by

$$
q(\mathcal{P})=\sum_{i, j} d^{2}\left(V_{i}, V_{j}\right) p_{i} p_{j}
$$

where $p_{i}=\left|V_{i}\right| /|V(G)|$. This function plays an important role in the proof of Szemerédi's regularity lemma and its variants.

The strong regularity lemma gives a regular partition $\mathcal{A}$, and a refinement $\mathcal{B}$ which is much more regular and is close to $\mathcal{A}$. For equitable partitions $\mathcal{A}$ and $\mathcal{B}$ with $\mathcal{B}$ a refinement of $\mathcal{A}$, the condition $\mathcal{B}$ is $\epsilon$-close to $\mathcal{A}$ is equivalent, up to a polynomial change in $\epsilon$, to $q(\mathcal{B}) \leq q(\mathcal{A})+\epsilon$. Indeed, if $\mathcal{B}$ is $\epsilon$-close to $\mathcal{A}$, then $q(\mathcal{B}) \leq q(\mathcal{A})+O(\epsilon)$, while if $q(\mathcal{B}) \leq q(\mathcal{A})+\epsilon$, then $\mathcal{B}$ is $O\left(\epsilon^{1 / 4}\right)$-close to $\mathcal{A}$. A version of this statement is present in Lemma 3.7 of [5]. As it is sufficicent and more convenient to work with mean square density instead of $\epsilon$-closeness, we do so from now on.

Note that in the strong regularity lemma, without loss of generality we may assume $f$ is a (monotonically) decreasing function. Indeed, this can be shown by considering the decreasing function $f^{\prime}(k):=\min _{1 \leq i \leq k} f(k)$. From the above discussion, it is easy to see that the strong regularity lemma has the following simple corollary, with a similar upper bound.

Corollary 1.1 Let $\epsilon>0$ and $f: \mathbb{N} \rightarrow(0,1)$ be a decreasing function. Then there exists a number $S=S(f, \epsilon)$ such that for every graph $G$ there are equitable partitions $\mathcal{A}, \mathcal{B}$ of the vertex set of $G$ with $|\mathcal{B}| \leq S, q(\mathcal{B}) \leq q(\mathcal{A})+\epsilon$, and $\mathcal{B}$ is $f(|\mathcal{A}|)$-regular.

We prove a lower bound for the strong regularity lemma of wowzer-type, which essentially matches the upper bound. Maybe surprisingly, our construction further shows that much less than what is required 
from the strong regularity lemma already gives wowzer-type bounds. In particular, even for Corollary 1.1. which appears considerably weaker than the strong regularity lemma, we get a wowzer-type lower bound. Note that in Corollary [1.1, $\mathcal{B}$ is not required to be a refinement of $\mathcal{A}$. In this case we could have $q(\mathcal{B})$ being close to $q(\mathcal{A})$ but the edge densities between the parts in these partitions are quite different from each other, i.e., these partitions are not close to each other.

Theorem 1.2 Let $0<\epsilon<2^{-100}$ and $f: \mathbb{N} \rightarrow(0,1)$ be a decreasing function with $f(1) \leq 2^{-100} \epsilon^{6}$. Define $W_{\ell}$ recursively by $W_{1}=1, W_{\ell+1}=T\left(2^{-70} \epsilon^{5} / f\left(W_{\ell}\right)\right)$, where $T$ is the tower function. Let $W=W_{t-1}$ with $t=2^{-20} \epsilon^{-1}$. Then there is a graph $G$ such that if equitable partitions $\mathcal{A}, \mathcal{B}$ of the vertex set of $G$ satisfy $q(\mathcal{B}) \leq q(\mathcal{A})+\epsilon$ and $\mathcal{B}$ is $f(|\mathcal{A}|)$-regular, then $|\mathcal{A}|,|\mathcal{B}| \geq W$.

We have the following corollary (by replacing $\epsilon$ by $\epsilon^{1 / 7}$ ), which is a simple to state lower bound of wowzer-type.

Corollary 1.2 For $0<\epsilon<2^{-700}$, there is a graph $G$ such that if equitable partitions $\mathcal{A}, \mathcal{B}$ of the vertex set of $G$ satisfy $|\mathcal{B}| \geq|\mathcal{A}|, q(\mathcal{B}) \leq q(\mathcal{A})+\epsilon$ and $\mathcal{B}$ is $\epsilon /|A|$-regular, then $|\mathcal{B}|,|\mathcal{A}|$ are bounded below by a function which is wowzer in $\Omega\left(\epsilon^{-1 / 7}\right)$.

\subsection{Induced graph removal}

Let $H$ be a fixed graph on $h$ vertices and let $G$ be a graph with $o\left(n^{h}\right)$ copies of $H$. To prove the graph removal lemma, we need to prove that all copies of $H$ can be removed from $G$ by deleting $o\left(n^{2}\right)$ edges. The standard approach is to apply the regularity lemma to the graph $G$ to obtain an $\epsilon$-regular vertex partition (with an appropriate $\epsilon$ ) into a constant number of parts $M(\epsilon)$. Then delete edges between pairs of parts $\left(V_{i}, V_{j}\right)$, including $i=j$, if the pair is not $\epsilon$-regular or the density between the pair is small. It is easy to see that there are few deleted edges. Furthermore, if there is a copy of $H$ in the remaining subgraph, then the edges go between pairs of parts which are $\epsilon$-regular and not of small density. A counting lemma then shows that in such a case the number of copies of $H$ is $\Omega\left(n^{h}\right)$ in the remaining subgraph, and hence in $G$ as well. But this would contradict the assumption that $G$ has $o\left(n^{h}\right)$ copies of $H$, so all copies of $H$ must already have been removed.

Recall that the induced graph removal lemma [5] is the analogous statement for induced subgraphs, and it is stronger than the graph removal lemma. It states that for any graph $H$ on $h$ vertices and $\epsilon>0$ there is $\delta=\delta(\epsilon, H)>0$ such that if a graph $G$ on $n$ vertices has at most $\delta n^{h}$ induced copies of $H$, then we can add or delete $\epsilon n^{2}$ edges of $G$ to obtain an induced $H$-free graph.

One well-known application of the induced graph removal lemma is in property testing. This is an active area of computer science where one wishes to quickly distinguish between objects that satisfy a property from objects that are far from satisfying that property. The study of this notion was initiated by Rubinfield and Sudan [35], and subsequently Goldreich, Goldwasser, and Ron [21] started the investigation of property testers for combinatorial objects. One simple consequence of the induced graph removal lemma is a constant time algorithm for induced subgraph testing with one-sided error (see [2] and its references). A graph on $n$ vertices is $\epsilon$-far from being induced $H$-free if at least $\epsilon n^{2}$ 
edges need to be added or removed to make it induced $H$-free. The induced graph removal lemma implies that there is an algorithm which runs in time $O_{\epsilon}(1)$ which accepts all induced $H$-free graphs, and rejects any graph which is $\epsilon$-far from being induced $H$-free with probability at least $2 / 3$. The algorithm samples $t=2 \delta^{-1} h$-tuples of vertices uniformly at random, where $\delta$ is picked according to the induced graph removal lemma, and accepts if none of them form an induced copy of $H$, and otherwise rejects. Any induced $H$-free graph is clearly accepted. If a graph is $\epsilon$-far from being induced $H$-free, then it contains at least $\delta n^{h}$ copies of $H$, and the probability that none of the sampled $h$-tuples forms an induced copy of $H$ is at most $(1-\delta)^{t}<1 / 3$. Notice that the running time as a function of $\epsilon$ depends on the bound in the induced graph removal lemma, and the proof using the strong regularity lemma gives a wowzer-type dependence.

It is tempting to try the same approach using Szemerédi's regularity lemma to obtain the induced graph removal lemma. However, there is a significant problem with this approach, which is handling the pairs between irregular pairs. To get around this issue, Alon, Fischer, Krivelevich, and Szegedy [5] developed the strong regularity lemma.

Because of its applications, including those in graph property testing, it has remained an intriguing problem to improve the bound in the induced graph removal lemma. This problem has been discussed in several papers by Alon and his collaborators [2], 6], [8]. The main result discussed in this subsection addresses this problem, improving the bound on the number of parts in the induced graph removal lemma from wowzer-type to tower-type. The tower function $t_{i}(x)$ is defined by $t_{0}(x)=x$ and $t_{i+1}(x)=$ $2^{t_{i}(x)}$. We say that $t_{i}(x)$ is a tower in $x$ of height $i$.

Theorem 1.3 For any graph $H$ on $h$ vertices and $0<\epsilon<1 / 2$ there is $\delta>0$ with $\delta^{-1}$ a tower in $h$ of height polynomial in $\epsilon^{-1}$ such that if a graph $G$ on $n$ vertices has at most $\delta n^{h}$ induced copies of $H$, then we can add or delete $\epsilon n^{2}$ edges of $G$ to obtain an induced $H$-free graph.

The following lemma is an easy corollary of the strong regularity lemma which was used in [5] to establish the induced graph removal lemma.

Lemma 1.2 For each $0<\epsilon<1 / 3$ and decreasing function $f: \mathbb{N} \rightarrow(0,1 / 3)$ there is $\delta^{\prime}=\delta^{\prime}(\epsilon, f)$ such that every graph $G=(V, E)$ with $|V| \geq \delta^{-1}$ has an equitable partition $V=V_{1} \cup \ldots \cup V_{k}$ and vertex subsets $W_{i} \subset V_{i}$ such that $\left|W_{i}\right| \geq \delta^{\prime}|V|$, each pair $\left(W_{i}, W_{j}\right)$ with $1 \leq i \leq j \leq k$ is $f(k)$-regular, and all but at most $\epsilon k^{2}$ pairs $1 \leq i \leq j \leq k$ satisfy $\left|d\left(V_{i}, V_{j}\right)-d\left(W_{i}, W_{j}\right)\right| \leq \epsilon$.

In fact, Lemma 1.2 is a little bit stronger than the original version in [5] in that each set $W_{i}$ is $f(k)$-regular with itself. The original version follows from the strong regularity lemma by taking the partition $V=V_{1} \cup \ldots \cup V_{k}$ to be the partiton $\mathcal{A}$ in the strong regularity lemma, and the subset $W_{i}$ to be a random part $V_{i j} \subset V_{i}$ of the refinement $\mathcal{B}$ of $\mathcal{A}$ in the strong regularity lemma.

From this slightly stronger version, the proof of the induced graph removal lemma is a bit simpler and shorter. Indeed, with $f(k)=\frac{1}{4 h} \epsilon^{h}$, which does not depend on $k$, if there is a mapping $\phi: V(H) \rightarrow$ $\{1, \ldots, k\}$ such that for all adjacent vertices $v, w$ of $H$, the edge density between $W_{\phi(v)}$ and $W_{\phi(w)}$ is at least $\epsilon$, and for all distinct nonadjacent vertices $v, w$ of $H$, the edge density between $W_{\phi(v)}$ and 
$W_{\phi(w)}$ is at most $1-\epsilon$, then a standard counting lemma shows that $G$ contains at least $\delta n^{h}$ induced copies of $H$, where $\delta=(\epsilon / 4)\left(\begin{array}{l}h \\ 2\end{array}\right) \delta^{\prime h}$. Hence, we may assume that there is no such mapping $\phi$. We then delete edges between $V_{i}$ and $V_{j}$ if the edge density between $W_{i}$ and $W_{j}$ is less than $\epsilon$, and one adds the edges between $V_{i}$ and $V_{j}$ if the density between $W_{i}$ and $W_{j}$ is more than $1-\epsilon$. The total number of edges added or removed is at most $5 \epsilon n^{2}$, and no induced copy of $H$ remains. Replacing $\epsilon$ by $\epsilon / 8$ in the above argument gives the induced graph removal lemma.

We find another proof of Lemma 1.2 with a better tower-type bound. This in turn implies, by the argument sketched above, the tower-type bound for the induced graph removal lemma stated in Theorem 1.3 .

The starting point for our approach to Lemma 1.2 is a weak regularity lemma due to Duke, Lefmann and Rödl [15]. This lemma says that for a $k$-partite graph, between sets $V_{1}, V_{2}, \ldots, V_{k}$, there is an $\epsilon$-regular partition of the cylinder $V_{1} \times \cdots \times V_{k}$ into a relatively small number of cylinders $K=$ $W_{1} \times \cdots \times W_{k}$, with $W_{i} \subset V_{i}$ for $1 \leq i \leq k$. The definition of an $\epsilon$-regular partition here is that all but an $\epsilon$-fraction of the $k$-tuples $\left(v_{1}, \ldots, v_{k}\right) \in V_{1} \times \cdots \times V_{k}$ are in $\epsilon$-regular cylinders, where a cylinder $W_{1} \times \cdots \times W_{k}$ is $\epsilon$-regular if all $\left(\begin{array}{c}k \\ 2\end{array}\right)$ pairs $\left(W_{i}, W_{j}\right), 1 \leq i<j \leq k$, are $\epsilon$-regular in the usual sense.

In the same way that one derives the strong regularity lemma from the ordinary regularity lemma, we show how to derive a strong version of this lemma. We will refer to this strengthening, of which Lemma 1.2 is a straightforward consequence, as the strong cylinder regularity lemma. It will also be convenient if, in this lemma, we make the requirement that a cylinder be regular slightly stronger, by asking that each $W_{i}$ be regular with itself. That is, we say that a cylinder $W_{1} \times \cdots \times W_{k}$ is strongly $\epsilon$-regular if all pairs $\left(W_{i}, W_{j}\right)$ with $1 \leq i, j \leq k$ are $\epsilon$-regular. A partition $\mathcal{K}$ of the cylinder $V_{1} \times \cdots \times V_{k}$ into cylinders $K=W_{1} \times \cdots \times W_{k}$, with $W_{i} \subset V_{i}$ for $1 \leq i \leq k$, is then said to strongly $\epsilon$-regular if all but an $\epsilon$-fraction of the $k$-tuples $\left(v_{1}, \ldots, v_{k}\right) \in V_{1} \times \cdots \times V_{k}$ are in strongly $\epsilon$-regular cylinders.

Let $P: V=V_{1} \cup \cdots \cup V_{k}$ be a partition of the vertex set of a graph and $\mathcal{K}$ be a partition of the cylinder $V_{1} \times \cdots \times V_{k}$ into cylinders. For each $K=W_{1} \times \cdots \times W_{k}$, with $W_{i} \subset V_{i}$ for $1 \leq i \leq k$, we let $V_{i}(K)=W_{i}$. We then define the partition $Q(\mathcal{K})$ of $V$ to be the refinement of $P$ which is the common refinement of all the parts $V_{i}(K)$ with $i \in[k]$ and $K \in \mathcal{K}$. The strong cylinder regularity lemma is now as follows.

Lemma 1.3 For $0<\epsilon<1 / 3$, positive integer $s$, and decreasing function $f: \mathbb{N} \rightarrow(0, \epsilon]$, there is $S=$ $S(\epsilon, s, f)$ such that the following holds. For every graph $G$, there is an integer $s \leq k \leq S$, an equitable partition $P: V=V_{1} \cup \ldots \cup V_{k}$ and a strongly $f(k)$-regular partition $\mathcal{K}$ of the cylinder $V_{1} \times \cdots \times V_{k}$ into cylinders satisfying that the partition $Q=Q(\mathcal{K})$ of $V$ has at most $S$ parts and $q(Q) \leq q(P)+\epsilon$. Furthermore, there is an absolute constant $c$ such that letting $s_{1}=s$ and $s_{i+1}=t_{4}\left(\left(s_{i} / f\left(s_{i}\right)\right)^{c}\right)$, we may take $S=s_{\ell}$ with $\ell=2 \epsilon^{-1}+1$.

In order to prove this lemma, we need, in addition to the Duke-Lefmann-Rödl regularity lemma, a lemma showing that for each $\epsilon>0$ there is $\delta>0$ such that every graph $G=(V, E)$ contains a vertex subset $U$ with $|U| \geq \delta|V|$ which is $\epsilon$-regular with itself, where, crucially, $\delta^{-1}$ is bounded above by a tower function of $\epsilon^{-1}$ of absolute constant height. While seemingly standard, we do not know of such a result in the literature. 
Lemma 1.2 follows from Lemma 1.3 by considering a random cylinder $K$ in the cylinder partition $\mathcal{K}$, with each cylinder picked with probability proportional to its size, and letting $W_{i}=V_{i}(K)$.

\subsection{Frieze-Kannan weak regularity lemma}

Frieze and Kannan [19], 20] developed a weaker notion of regularity which is sufficient for certain applications and for which the dependence on the approximation $\epsilon$ is much better. It states the existence of a vertex partition into a small number of parts for which the number of edges across any two vertex subsets is within $\epsilon n^{2}$ of what is expected based on the edge densities between the parts of the partition and the intersection sizes of the vertex subsets with these parts.

Lemma 1.4 (Frieze-Kannan weak regularity lemma) For each $\epsilon>0$ there is a positive integer $k(\epsilon)$ such that every graph $G=(V, E)$ has an equitable vertex partition $V=V_{1} \cup \ldots \cup V_{k}$ with $k \leq k(\epsilon)$ satisfying that for all subsets $A, B \in V$, we have

$$
\left|e(A, B)-\sum_{1 \leq i, j \leq k} d\left(V_{i}, V_{j}\right)\right| A \cap V_{i}|| B \cap V_{j}|| \leq \epsilon|V|^{2} .
$$

The weak regularity lemma has a number of algorithmic applications. Frieze and Kannan [20] used the weak regularity lemma to give constant-time approximation algorithms for some general problems in dense graphs, a special case being the Max-Cut of a graph. Recently, Bansal and Williams [12] used the weak regularity lemma to obtain a faster combinatorial algorithm for Boolean matrix multiplication. The importance of the weak regularity lemma is further discussed in the citation of the recent Knuth Prize to Kannan.

As there are several applications of the weak regularity lemma to fundamental algorithmic problems, we would like to know the correct bounds on the number of parts for the weak regularity lemma. The proof of the weak regularity lemma [20] shows that we may take $k(\epsilon)=2^{O\left(\epsilon^{-2}\right)}$. If this upper bound could be improved, it would lead to faster algorithms for several problems of interest. Lovász and Szegedy [29] studied the problem of estimating the minimum number of parts $k(\epsilon)$ required for the weak regularity lemma, proving a lower bound on $k(\epsilon)$ of the form $2^{\Omega\left(\epsilon^{-1}\right)}$. Here we close the gap by proving a new lower bound which matches the upper bound.

Theorem 1.4 For each $\epsilon>0$, there are graphs for which the minimum number of parts in a weak regular partition with approximation $\epsilon$ is $2^{\Omega\left(\epsilon^{-2}\right)}$.

A careful analysis of the proof of Theorem 1.4 shows that the number of parts required in the weak regularity lemma with approximation $\epsilon$ is at least $2^{-2^{-60} \epsilon^{-2}}$ for $0<\epsilon \leq 2^{-50}$. In fact, the theorem yields a stronger result, since we do not here require that the partition be equitable.

While the number of parts in the weak regularity lemma is $2^{\Theta\left(\epsilon^{-2}\right)}$, the proof obtains the partition as an overlay of only $O\left(\epsilon^{-2}\right)$ sets. As discussed in [29], in some applications, such as in [4], this can be treated as if there were only about $O\left(\epsilon^{-2}\right)$ classes, which makes the weak regularity lemma quite efficient. It was shown in [4, and is also implied by Theorem 1.4, that the partition cannot be the overlay of fewer sets. 


\subsection{The regular approximation lemma}

Another strengthening of Szemerédi's regularity lemma came from the study of graph limits by Lovász and Szegedy [29], and also from work on the hypergraph generalization of the regularity lemma by Rödl and Schacht 32]. This regularity lemma, known as the regular approximation lemma [33, provides an arbitrary precision for the regularity as a function of the number of parts of the partition if an $\epsilon$-fraction of the edges are allowed to be added or removed.

For a function $g: \mathbb{N} \rightarrow(0,1)$, a partition of the vertex set into $k$ parts is $g$-regular if all pairs of distinct parts in the partition are $g(k)$-regular.

Lemma 1.5 (Regular approximation lemma) For every $\epsilon>0$, positive integer $s$ and decreasing function $g: \mathbb{N} \rightarrow(0,1)$, there is an integer $T=T(g, \epsilon, s)$ so that given a graph $G$ with $n$ vertices, one can add-to/remove-from $G$ at most $\epsilon n^{2}$ edges and thus get a graph $G^{\prime}$ that has a g-regular equitable partition of order $k$ for some $s \leq k \leq T$.

Lovász and Szegedy [29] state that the regular approximation lemma is equivalent to the strong regularity lemma, Lemma 1.1. It is not difficult to deduce Lemma 1.5 from the strong regularity lemma, see [9] or [33] for details. Unlike the original graph limit approach, this proof of the regular approximation lemma gives explicit bounds and yields a polynomial time algorithm for finding the partition and the necessary edge modifications. In the other direction, by applying Lemma 1.5 with $1 / g$ a tower in the $1 / f$ from Lemma 1.1, letting $\mathcal{A}$ be the $g$-regular partition of $G^{\prime}$, and then using Szemerédi's regularity lemma to get a refinement $\mathcal{B}$ of $\mathcal{A}$ which is an $f(\mathcal{A})$-regular partition of $G$, it is easy to deduce the strong regularity lemma.

The major caveat here is the additional use of Szemerédi's regularity lemma in deducing the strong regularity lemma from the regular approximation lemma. Due to the additional use of Szemerédi's regularity lemma, it does not rule out the possibility that the wowzer-type upper bound on $T$ in the regular approximation lemma can be improved to tower-type. Maybe surprisingly, we indeed make such an improvement.

Theorem 1.5 For $\epsilon>0$, positive integer $s$ and a decreasing function $g: \mathbb{N} \rightarrow(0,1)$, let $\delta(t)=$ $\min \left(\frac{g(t)^{3}}{32 t^{2}}, \epsilon / 2\right)$. Let $t_{1}=s$ and for $i \geq 1$ let $t_{i+1}=t_{i} k\left(\delta\left(t_{i}\right)\right)$, where $k$ is as in the weak regularity lemma, so $k(\alpha)=2^{O\left(\alpha^{-2}\right)}$. Let $T_{0}=t_{j}$ with $j=4 \epsilon^{-2}$. Then the regular approximation lemma holds with $T=16 T_{0} / \delta\left(T_{0}\right)^{2}$. In other words, the regular approximation lemma holds with a tower-type bound.

It is usually the case that $1 / g(t)$ in the regular approximation lemma is at most a tower of constant height in $\epsilon^{-1}$ and $t$, and in this case the upper bound $T$ on the number of parts is only a tower of height polynomial in $\epsilon^{-1}$. Only in the unusual case of $1 / g$ being of tower-type growth does the number of parts needed in the regular approximation lemma grow as wowzer-type.

Alon, Shapira, and Stav [9] give a proof of the regular approximation lemma which yields a polynomial time algorithm for finding the partition and the necessary edge modifications. Similarly, our new proof 
can be made algorithmic with a polynomial time algorithm for finding the partition and the necessary edge modifications. Making the proof algorithmic is essentially the same as done in [9], so we do not include the details.

A partition of a graph satisfying the weak regularity lemma, Lemma 1.4, is called a weak $\epsilon$-regular partition. Tao showed [41] (see also [33]), by iterating the weak regularity lemma, that one obtains the following regularity lemma which easily implies Szemerédi's regularity lemma with the usual towertype bounds.

Lemma 1.6 For all $\epsilon>0$, positive integers $s$ and functions $\delta: \mathbb{N} \rightarrow(0,1)$, there is a $T_{0}$ such that every graph has an equitable vertex partition $P$ into $t \geq s$ parts which is weak $\epsilon$-regular, an equitable vertex refinement $Q$ into at most $T_{0}$ parts which is weak $\delta(t)$-regular, and $q(Q) \leq q(P)+\epsilon$.

Let $t_{1}=s$, and for $i \geq 1$, let $t_{i+1}=t_{i} k\left(\delta\left(t_{i}\right)\right)$, where $k$ is as in the weak regularity lemma. Recall $k(\epsilon)$ is exponential in $\epsilon^{-2}$. Then $T_{0}$ in Lemma 1.6 is given by $T_{0}=t_{j}$ with $j=\epsilon^{-1}$. In particular, if $\delta^{-1}$ is bounded above by a tower of constant height, then $T_{0}$ in Tao's regularity lemma grows as a tower of height linear in $\epsilon^{-1}$.

Our proof of Theorem 1.5 shows that the regular approximation lemma is equivalent to Tao's regularity lemma with similar bounds. In fact, we show that $T$ in the regular approximation lemma can be taken to be $T=16 T_{0} / \delta\left(T_{0}\right)^{2}$, where $T_{0}=T_{0}\left(\delta, \epsilon_{0}, s\right)$ is the bound on the number of parts in Tao's regularity lemma, $\delta(t)=\min \left(\frac{g(t)^{3}}{32 t^{2}}, \epsilon / 2\right)$, and $\epsilon_{0}=(\epsilon / 2)^{2}$. As Tao's regularity lemma is a simple consequence of the regular approximation lemma and an application of the weak regularity lemma, it suffices to show how to deduce the regular approximation lemma from Tao's regularity lemma.

The proof starts by applying Tao's regularity lemma with $\delta$ and $\epsilon_{0}$ as above. For each pair $(X, Y)$ of parts in $Q$, where $X \subset A$ and $Y \subset B$ with $A, B$ parts of $P$, we randomly add/delete edges between $X, Y$ with a certain probability so that the density between $X$ and $Y$ is about the same as the density between $A$ and $B$. We show that in doing this we have made every pair $(A, B)$ of parts of $P g(t)$ regular with $t=|P|$. Since $q(Q) \leq q(P)+\epsilon_{0}$, the edge density $d(X, Y)$ between most pairs $(X, Y)$ of parts of $Q$ is close to the edge density $d(A, B)$ between $A$ and $B$, and few edges are changed to obtain a graph $G^{\prime}$ for which the partition $P$ is $g$-regular.

We next briefly discuss lower bounds for the regular approximation lemma. In the case $g$ is a (small) constant function, a tower-type lower bound follows from Theorem 1.1. If $g$ is at least a tower function, we get a lower bound of wowzer-type from Theorem 1.2 and the fact that the strong regularity lemma follows from the regularity approximation lemma with an additional application of Szemerédi's regularity lemma as discussed earlier. One could likely come up with a construction giving a general lower bound essentially matching Theorem 1.5, but as the already mentioned interesting cases discussed above are handled by Theorems 1.1 and 1.2, we do not include such a construction.

\section{Organization}

In the next section, we prove some useful tools for establishing lower bounds for Szemerédi's regularity lemma and the strong regularity lemma. In Section 3, we give a general construction and use it to prove Theorem 1.1 which addresses questions of Szemerédi and Gowers on the number of irregular 
pairs in Szemerédi's regularity lemma. In Section 4, we use the general construction to prove Theorem 1.2. which gives a wowzer-type lower bound on the number of parts of the two partitions in the strong regularity lemma. In Section 5, we prove the strong cylinder regularity lemma and use it to prove a tower-type upper bound on the induced graph removal lemma. In Section 6, we prove a tower-type upper bound on the number of parts in the regular approximation lemma. In Section 7, we prove a tight lower bound on the number of parts in the weak regularity lemma. These later sections, Sections 5. 6 and 7, are largely independent of earlier sections and of each other. The interested reader may therefore skip forward without fear of losing the thread.

We finish with some concluding remarks. This includes a discussion showing that in the regularity lemma, the condition that the parts in the partition are of equal size does not affect the bounds by much. We also discuss an early version of Szemerédi's regularity lemma, and a recent result of Malliaris and Shelah which shows an interesting connection between irregular pairs in the regularity lemma and the appearance of half-graphs.

Throughout the paper, we systematically omit floor and ceiling signs whenever they are not crucial for the sake of clarity of presentation. We also do not make any serious attempt to optimize absolute constants in our statements and proofs.

\section{Tools}

Suppose $S=S_{1}+\cdots+S_{n}$ is the sum of $n$ mutually independent random variables, where for each $i$, $\operatorname{Pr}\left[S_{i}=1\right]=p$ and $\operatorname{Pr}\left[S_{i}=0\right]=1-p$. The sum $S$ has a binomial distribution with parameters $p$ and $n$, and has expected value pn. A Chernoff-type estimate (see Theorem A.1.4 in [10]) implies that for $a>0$,

$$
\operatorname{Pr}[S-p n>a]<e^{-2 a^{2} / n}
$$

By symmetry, we also have $\operatorname{Pr}[S-p n<-a]<e^{-2 a^{2} / n}$ and hence $\operatorname{Pr}[|S-p n|>a]<2 e^{-2 a^{2} / n}$.

We start by proving a couple of lemmas on the edge distribution of random bipartite graphs with different part sizes. Consider the random bipartite graph $B=B(m, M)$ with parts $[m]$ and $[M]$ formed by each vertex $i \in[m]$ having exactly $M / 2$ neighbors (we assume $M$ is even) in $[M]$ picked uniformly at random and independently of the choices of the neighborhoods for the other vertices in $[m]$.

The following lemma shows that, with high probability, certain simple estimates on the number of common neighbors or nonneighbors of any two vertices in $B(m, M)$ hold.

Lemma 2.1 Let $M \geq m$ be positive integers with $M \geq 2^{20}$ even, and $0<\mu<1 / 2$ be such that $m \geq 2 \mu^{-2} \log M$. Then, with probability at least $1-M^{-2}$, the random bipartite graph $B=B(m, M)$ has the following properties:

- for any distinct $j, j^{\prime} \in[M]$, the number of $i$ for which $j$ and $j^{\prime}$ are either both neighbors of $i$ or both nonneighbors of $i$ is less than $\left(\frac{1}{2}+\mu\right) m$. 
- for any distinct $i, i^{\prime} \in[m]$, the number of common neighbors of $i$ and $i^{\prime}$ and the number of common nonneighbors of $i$ and $i^{\prime}$ in $[M]$ are both less than $\left(\frac{1}{4}+M^{-1 / 4}\right) M$.

Proof: Fix distinct $j, j^{\prime} \in[M]$. For each $i \in[m]$, the probability that $i$ is adjacent to both $j, j^{\prime}$ or nonadjacent to both $j, j^{\prime}$ is $\left(\frac{M}{2}-1\right) /(M-1)<\frac{1}{2}$, and these events are independent of each other. Therefore, by (1), the probability that the number of $i$ for which $j$ and $j^{\prime}$ are either both neighbors of $i$ or both nonneighbors of $i$ is at least $\left(\frac{1}{2}+\mu\right) m$ is at most $e^{-2(\mu m)^{2} / m}=e^{-2 \mu^{2} m} \leq M^{-4}$. As there are $\left(\begin{array}{c}M \\ 2\end{array}\right)$ choices for $j, j^{\prime}$, and $\frac{1}{2} M^{-2} \geq M^{-4}\left(\begin{array}{c}M \\ 2\end{array}\right)$, by the union bound we have that $B$ has the first desired property with probability at least $1-\frac{1}{2} M^{-2}$.

As the hypergeometric distribution is at least as concentrated as the corresponding binomial distribution (for a proof, see Section 6 of [25]), we can apply (1) to conclude that for each fixed pair $i, i^{\prime} \in[m]$ of distinct vertices the probability that the number of common neighbors of $i$ and $i^{\prime}$ is at least $\left(\frac{1}{4}+M^{-1 / 4}\right) M$ is at most $e^{-2\left(M^{-1 / 4} M\right)^{2} / M}=e^{-2 M^{1 / 2}}$. Similarly, for each fixed pair $i, i^{\prime} \in[m]$ of distinct vertices the probability that the number of common nonneighbors of $i$ and $i^{\prime}$ in $[M]$ is at least $\left(\frac{1}{4}+M^{-1 / 4}\right) M$ is at most $e^{-2 M^{1 / 2}}$.

As there are $\left(\begin{array}{c}m \\ 2\end{array}\right)$ choices for $i, i^{\prime}$ and $\frac{1}{2} M^{-2} \geq 2 e^{-2 M^{1 / 2}}\left(\begin{array}{c}m \\ 2\end{array}\right)$, by the union bound we have that $B$ has the second desired property with probability at least $1-\frac{1}{2} M^{-2}$. Hence, with probability at least $1-M^{-2}, B$ has both desired properties, which completes the proof.

The next lemma shows that the edges in $B(m, M)$ are almost surely uniformly distributed between large vertex subsets.

Lemma 2.2 Let $M$ and $m$ be positive integers with $M$ even. With probability at least $1-M^{-1}$, for any $U_{1} \subset[m]$ and $U_{2} \subset[M]$ with $\left|U_{1}\right|=u_{1}$ and $\left|U_{2}\right|=u_{2}$, we have

$$
\left|e_{B}\left(U_{1}, U_{2}\right)-\frac{1}{2} u_{1} u_{2}\right| \leq \sqrt{f}
$$

where

$$
f=f\left(u_{1}, u_{2}\right)=u_{1} u_{2}\left(u_{1} \ln \frac{e m}{u_{1}}+u_{2} \ln \frac{e M}{u_{2}}\right)
$$

Proof: For fixed subsets $U_{1} \subset[m]$ and $U_{2} \subset[M]$, the random variable $e_{B}\left(U_{1}, U_{2}\right)$, which has mean $\frac{1}{2}\left|U_{1}\right|\left|U_{2}\right|$, despite not satisfying a binomial distribution, still satisfies the estimate (1) for the corresponding binomial distribution with parameters $1 / 2$ and $\left|U_{1}\right|\left|U_{2}\right|$. Indeed, note that $e_{B}\left(U_{1}, U_{2}\right)$ is the sum of the degrees of the vertices of $U_{1}$ in $U_{2}$, and these $\left|U_{1}\right|$ degrees are identical independent random variables, each satisfying a hypergeometric distribution. By Theorem 4 in Section 6 of [25], the expected value of the exponential of a random variable with a hypergeometric distribution is at most the expected value of the exponential of the random variable with the corresponding binomial distribution. Substituting this estimate into the proof of (10) shows that the Chernoff estimate also holds for $e_{B}\left(U_{1}, U_{2}\right)$. Hence, the probability (2) doesn't hold for a particular pair $U_{1}, U_{2}$ is less than 
$2 e^{-2 f /\left(u_{1} u_{2}\right)}$. By the union bound, the probability that there is a pair of subsets $U_{1} \subset[m]$ and $U_{2} \subset[M]$ not satisfying (2) is at most

$$
\begin{aligned}
\sum_{u_{1}=1}^{m} \sum_{u_{2}=1}^{M}\left(\begin{array}{l}
m \\
u_{1}
\end{array}\right)\left(\begin{array}{l}
M \\
u_{2}
\end{array}\right) 2 e^{-2 f /\left(u_{1} u_{2}\right)} & \leq \sum_{u_{1}=1}^{m} \sum_{u_{2}=1}^{M}\left(\frac{e m}{u_{1}}\right)^{u_{1}}\left(\frac{e M}{u_{2}}\right)^{u_{2}} 2 e^{-2 f /\left(u_{1} u_{2}\right)} \\
& =\sum_{u_{1}=1}^{m} \sum_{u_{2}=1}^{M} 2\left(\frac{e m}{u_{1}}\right)^{-u_{1}}\left(\frac{e M}{u_{2}}\right)^{-u_{2}} \leq M^{-1}
\end{aligned}
$$

From the bipartite graph $B$, we construct equitable partitions $\left(A_{i}, B_{i}\right)_{i=1}^{m}$ of $[M]$, by letting $A_{i}$ denote the set of neighbors of vertex $i \in[m]$ in graph $B$. From Lemmas 2.1 and 2.2, we have the following corollary.

Corollary 2.1 Suppose $M \geq m$ are positive integers with $M \geq 2^{20}$ even, and $0<\mu<1 / 2$ is such that $m \geq 2 \mu^{-2} \log M$. There is a bipartite graph $B$ with parts $[m]$ and $[M]$, with each vertex in $[m]$ of degree $M / 2$ with the following properties. The estimate (2) holds for all $U_{1} \subset[m]$ and $U_{2} \subset[M]$ with $\left|U_{1}\right|=u_{1}$ and $\left|U_{2}\right|=u_{2}$, and $B$ satisfies the two properties in the conclusion of Lemma 2.1.

The next lemma is a useful consequence of the equitable partitions $\left(A_{i}, B_{i}\right)_{i=1}^{m}$ behaving randomly. Given a vector $\lambda \in \mathbb{R}^{M}$ and $1 \leq q<\infty$, write $\|\lambda\|_{q}$ for $\left(\sum_{i=1}^{M}\left|\lambda_{i}\right|^{q}\right)^{1 / q}$ and $\|\lambda\|_{\infty}$ for $\max _{1 \leq i \leq M}\left|\lambda_{i}\right|$.

Lemma 2.3 Let $M$ be a positive even integer, $0<\mu<1 / 2$, and $\left(A_{i}, B_{i}\right)_{i=1}^{m}$ be a sequence of partitions satisfying the conclusion of Corollary 2.1. Suppose that $0<\sigma, \tau, \alpha$ are such that $\sigma, \tau<1, \alpha<1 / 2$, and

$$
\left(\frac{1}{2}-\mu\right)\left(1-\sigma^{2}\right)>\frac{\tau}{2}+2(1-\tau) \alpha(1-\alpha)
$$

Then for every sequence $\lambda=\left(\lambda_{1}, \ldots, \lambda_{M}\right)$ of nonnegative real numbers which are not all zero with $\|\lambda\|_{2}=\sigma\|\lambda\|_{1}$, there are at least $\tau m$ values of $i$ for which $\min \left(a_{i}, b_{i}\right)>\alpha\|\lambda\|_{1}$, where $a_{i}=\sum_{j \in A_{i}} \lambda_{j}$ and $b_{i}=\sum_{j \in B_{i}} \lambda_{j}$.

Proof: Note that by multiplying each $\lambda_{j}$ by $1 /\|\lambda\|_{1}$, we may assume without loss of generality that $\|\lambda\|_{1}=1$. For distinct $j, j^{\prime} \in[M]$, let $\left(j, j^{\prime}\right)_{i}$ denote that $j$ and $j^{\prime}$ lie in different sets in the partition $\left(A_{i}, B_{i}\right)$. Since for any distinct $j, j^{\prime} \in[M]$, the number of $i$ for which $\left(j, j^{\prime}\right)_{i}$ holds is at least $\left(\frac{1}{2}-\mu\right) m$, we have

$$
\sum_{\left(j, j^{\prime}\right)_{i}} \lambda_{j} \lambda_{j^{\prime}} \geq\left(\frac{1}{2}-\mu\right) m \sum_{j} \lambda_{j}\left(1-\lambda_{j}\right)=\left(\frac{1}{2}-\mu\right) m\left(\|\lambda\|_{1}-\|\lambda\|_{2}^{2}\right)=\left(\frac{1}{2}-\mu\right) m\left(1-\sigma^{2}\right),
$$

where the sum is over all ordered triples $\left(j, j^{\prime}, i\right)$ with $j, j^{\prime}$ distinct and $j$ and $j^{\prime}$ lie in different sets in the partition $\left(A_{i}, B_{i}\right)$. We have the identity

$$
\sum_{\left(j, j^{\prime}\right)_{i}} \lambda_{j} \lambda_{j^{\prime}}=2 \sum_{i} a_{i} b_{i}
$$


Since $a_{i}+b_{i}=1$, we have $a_{i} b_{i} \leq 1 / 4$ and if $\min \left(a_{i}, b_{i}\right) \leq \alpha$, then $a_{i} b_{i} \leq \alpha(1-\alpha)$. So if $\min \left(a_{i}, b_{i}\right) \leq \alpha$ for all but less than $\tau m$ values of $i$, then

$$
\sum_{\left(j, j^{\prime}\right)_{i}} \lambda_{j} \lambda_{j^{\prime}}<\frac{\tau}{2} m+2(1-\tau) m \alpha(1-\alpha) .
$$

Comparing with (3) and dividing by $m$, this contradicts the supposition, and completes the proof.

As usual, $G(n, p)$ denotes the random graph on $n$ vertices chosen by picking each pair of vertices as an edge randomly and independently with probability $p$. We finish this section with a few standard lemmas on the edge distribution in $G(n, p)$.

Lemma 2.4 In $G(n, p)$, with probability at least $1-n^{-2}$, every pair of disjoint vertex subsets $U_{1}$ and $U_{2}$ satisfy

$$
\left|e\left(U_{1}, U_{2}\right)-p u_{1} u_{2}\right| \leq \sqrt{g},
$$

where $u_{1}=\left|U_{1}\right|, u_{2}=\left|U_{2}\right|$ and, for $u_{1} \leq u_{2}, g=g\left(u_{1}, u_{2}\right)=2 u_{1} u_{2}^{2} \ln \frac{n e}{u_{2}}$.

Proof: For fixed sets $U_{1}$ and $U_{2}$, the quantity $e\left(U_{1}, U_{2}\right)$ is a binomial distributed random variable with parameters $u_{1} u_{2}$ and $p$. By (1), we have that the probability (4) does not hold is less than $2 e^{-2 g /\left(u_{1} u_{2}\right)}$. By the union bound, the probability that there are disjoint sets $U_{1}$ and $U_{2}$ for which (4) does not hold is at most

$$
\begin{aligned}
\sum_{u_{2}=1}^{n} \sum_{u_{1}=1}^{u_{2}}\left(\begin{array}{c}
n \\
u_{2}
\end{array}\right)\left(\begin{array}{c}
n-u_{2} \\
u_{1}
\end{array}\right) 2 e^{-2 g /\left(u_{1} u_{2}\right)} & \leq \sum_{u_{2}=1}^{n} \sum_{u_{1}=1}^{u_{2}}\left(\frac{n e}{u_{2}}\right)^{u_{2}}\left(\frac{n e}{u_{1}}\right)^{u_{1}} 2 e^{-2 g /\left(u_{1} u_{2}\right)} \\
& \leq \sum_{u_{2}=1}^{n} \sum_{u_{1}=1}^{u_{2}} 2\left(\frac{n e}{u_{2}}\right)^{-2 u_{2}} \leq n^{-2} .
\end{aligned}
$$

The result follows.

Lemma 2.5 In $G(n, p)$, with probability at least $1-n^{-2}$, every vertex subset $U$ satisfies

$$
\left|e(U)-p\left(\begin{array}{l}
u \\
2
\end{array}\right)\right| \leq \sqrt{g}
$$

where $u=|U|$ and $g=g(u)=\frac{1}{2} u^{3} \ln \frac{n e}{u}$.

Proof: For fixed $U$, the quantity $e(U)$ is a binomially distributed random variable with parameters

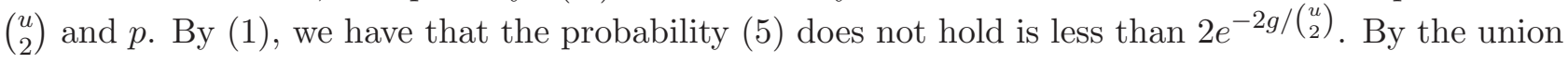
bound, the probability that there is a vertex subset $U$ for which (5) does not hold is at most

$$
\sum_{u=2}^{n}\left(\begin{array}{l}
n \\
u
\end{array}\right) 2 e^{-2 g /\left(\begin{array}{l}
u \\
2
\end{array}\right)} \leq \sum_{u=2}^{n}\left(\frac{n e}{u}\right)^{u} 2 e^{-2 g /\left(\begin{array}{l}
u \\
2
\end{array}\right)} \leq 2 \sum_{u=2}^{n}\left(\frac{n e}{u}\right)^{-(u+1)} \leq n^{-2} .
$$

Combining the estimates from the previous two lemmas, we can bound the probability in $G(n, p)$ that there are two not necessarily disjoint subsets with large edge discrepancy between them. 
Lemma 2.6 In $G(n, p)$, the probability that there are integers $u_{1}$ and $u_{2}$ with $u_{1} \leq u_{2}$ and not necessarily disjoint vertex subsets $U_{1}$ and $U_{2}$ with $\left|U_{1}\right|=u_{1}$ and $\left|U_{2}\right|=u_{2}$ such that

$$
\left|e\left(U_{1}, U_{2}\right)-p u_{1} u_{2}\right|>5 \sqrt{h},
$$

where $h=h\left(u_{1}, u_{2}\right)=u_{1} u_{2}^{2} \ln \frac{n e}{u_{2}}$, is at most $2 n^{-2}$.

Proof: For sets $U_{1}$ and $U_{2}$, letting $U_{1}^{\prime}=U_{1} \backslash U_{2}, U_{2}^{\prime}=U_{2} \backslash U_{1}$, and $U=U_{1} \cap U_{2}$, we have

$$
e\left(U_{1}, U_{2}\right)=e\left(U_{1}^{\prime}, U_{2}\right)+2 e(U)+e\left(U, U_{2}^{\prime}\right)
$$

We have that the bounds in Lemmas 2.4 and 2.5 hold with probability at least $1-2 n^{-2}$. Hence, using the triangle inequality, and $\left|U_{1}^{\prime}\right| \leq u_{1},|U| \leq u_{1},\left|U_{2}^{\prime}\right| \leq u_{2}$, we have

$$
\left|e\left(U_{1}, U_{2}\right)-p u_{1} u_{2}\right| \leq 2 \sqrt{g\left(u_{1}, u_{2}\right)}+2 \sqrt{g\left(u_{1}\right)}+p u_{1} \leq 5 \sqrt{h}
$$

with probability at least $1-2 n^{-2}$. Here the extra $p u_{1}$ factor comes from the fact that degenerate edges are not counted in $e(U)$.

\section{A general graph construction}

In this section, we will define a nonuniform random graph $G=(V, E)$ which, assuming certain estimates, has the property that any sufficiently regular partition of its vertex set is close to being a refinement of a particular partition of $G$ into many parts. As this particular partition has many parts, this will imply that any sufficiently regular partition will have many parts. After defining $G$, we will prove that certain useful estimates on the edge distribution of $G$ hold with positive probability. We will use these estimates to show that $G$ has the desired property.

\subsection{Defining graph $G$}

Following Gowers [22], we attempt to reverse engineer the proof of Szemerédi's regularity lemma to show that the upper bound is essentially best possible. The proof of the regularity lemma follows a sequence of refinements of the vertex set of the graph until we arrive at a regular partition, with the number of parts in each partition exponentially larger than in the previous partition. We build a sequence of partitions of the vertex set, and then describe how the edges of $G$ are distributed between the various parts of the partition. To show that any (sufficiently) regular partition $\mathcal{Z}$ of $V(G)$ requires many parts, we show that $\mathcal{Z}$ is roughly a refinement of the partitions we constructed in $\operatorname{defining} G$.

Let $m_{1} \geq 2^{200}$ be a positive integer and $\rho=2^{-20}$. For $2 \leq i \leq s$, let $m_{i}=m_{i-1} a_{i-1}$, where $a_{i-1}=2^{\left\lfloor\rho m_{i-1}^{9 / 10}\right\rfloor}$. Suppose $p_{i} \geq m_{i}^{-1 / 10}$ for $1 \leq i \leq s-1$.

The vertex set $V$ has a sequence of equitable partitions $P_{1}, \ldots, P_{s}$, where $P_{j}$ is a refinement of $P_{i}$ for $j>i$ defined as follows. The number of parts of $P_{i}$ is $m_{i}$. For each set $X$ in partition $P_{i}$, we pick 
an equitable partition of $X$ into $a_{i}$ parts, and let $P_{i+1}$ be the partition of $V$ with $m_{i+1}=m_{i} a_{i}$ parts consisting of the union of these partitions of parts of $P_{i}$.

For $1 \leq i \leq s-1$, let $G_{i}$ be a uniform random graph on $P_{i}$ with edge probability $p_{i}$. That is, the vertices of the graph are the $m_{i}$ pieces of the partition and we place edges independently with probability $p_{i}$. In practice, we will make certain specific assumptions about the edge distribution of $G_{i}$ but these will hold with high probability in a random graph. For example, we shall assume that every vertex in $G_{i}$ has degree at least $p_{i} m_{i} / 2$.

For each $X, Y \in P_{i}$ with $(X, Y)$ an edge of $G_{i}$, we have an equitable partition $Q_{X Y}: X=X_{Y}^{1} \cup X_{Y}^{2}$ into two parts, where $X_{Y}^{j}$ is a union of some of the parts in $P_{i+1}$ for $j=1,2$. For each $X \in P_{i}$, we shall choose the partitions $Q_{X Y}$ with $Y$ adjacent to $X$ in $G_{i}$ to satisfy the properties of Corollary 2.1 with

$\mu=2 \rho^{1 / 2}=2^{-9}$. Note that this is possible since we are taking $M=a_{i}$ and $m \geq p_{i} m_{i} / 2 \geq m_{i}^{9 / 10} / 2$, so $m \geq 2 \mu^{-2} \log M$, as required.

We finish the construction of $G$ by defining which pairs of vertices are adjacent. Vertices $u, v \in V$ are adjacent in $G$ if there is $i, 1 \leq i \leq s-1$, an edge $(X, Y)$ of $G_{i}$, and $j \in\{1,2\}$ with $u \in X_{Y}^{j}, v \in Y_{X}^{j}$. An equivalent way of defining the graph $G$ is as follows. For $1 \leq j<i$, let $G_{j, i}$ denote the graph with vertex set $P_{i}$, where $X, Y \in P_{i}$ is an edge of $G_{j, i}$ if there are $X^{\prime}, Y^{\prime} \in P_{j}$ that are adjacent in $G_{j}$, and $d \in\{1,2\}$ with $X \subset X_{Y^{\prime}}^{\prime d}$ and $Y \subset Y_{X^{\prime}}^{\prime d}$. For $1<i \leq s$, let $G^{i}$ denote the graph on $P_{i}$ whose edge set is the union of the edge sets of $G_{1, i}, \ldots, G_{i-1, i}$. Finally, two vertices $u, v \in V$ are adjacent in $G$ if there is an edge $(X, Y)$ of $G^{s}$ with $u \in X$ and $v \in Y$. Note that $G^{1}$ is simply the empty graph on $P_{1}$.

We say that a subset $Z \beta$-overlaps another set $X$ if $|X \cap Z| \geq \beta|Z|$, that is, if a $\beta$-fraction of $Z$ is in $X$. A set $Z$ is $\beta$-contained in a partition $P$ of $V$ if there is a set $X \in P$ such that $Z \beta$-overlaps $X$.

An equitable partition $\mathcal{Z}$ of $V$ is a $(\beta, v)$-refinement of a partition $P$ of $V$ if, for at least $(1-v)|\mathcal{Z}|$ sets $Z \in \mathcal{Z}$, the set $Z$ is $(1-\beta)$-contained in $P$. In particular, when $\beta=v=0$, this notion agrees with the standard notion of refinement. That is, $\mathcal{Z}$ is a refinement of $P$ is equivalent to $\mathcal{Z}$ being a (0,0)-refinement of $P$.

Our main result, from which Theorem 1.1 easily follows, now says that for an appropriate choice of $p_{i}$, every regular partition of $G$ must be close to a refinement of $P_{s-1}$. In the proof of Theorem 1.1. Theorem 3.1 will be used only in the case $a=s-1$. However, for the lower bound on the strong regularity lemma in Theorem 1.2, we will need to apply Theorem 3.1 for various values of $a$. This is why the parameter $a$ is introduced.

Theorem 3.1 Let $\nu=3 \sum_{i=1}^{s-1} p_{i}$, and suppose $p_{i}>2^{10} \eta m_{1}^{2}$ for $1 \leq i \leq a, 1-2^{7} \nu>\epsilon, \beta=20 m_{1}^{-3 / 2}$, $\delta<\beta / 4$, and $v=5 m_{1}^{-1 / 2}$. With positive probability, the random graph $G$ has the following property. Every $(\epsilon, \delta, \eta)$-regular equitable partition of $G$ is a $(\beta, v)$-refinement of $P_{a}$.

\subsection{Edge distribution in $G$}

Having defined the (random) graph $G$, we now show that with positive probability $G$ satisfies certain properties (see Lemma 3.11) concerning its edge distribution which we will use to prove Theorem 1.1 . Note that $G$ is determined by the $G_{i}$. For some of the desired properties, it will be enough to show 
that the edges in each $G_{i}$ are sufficiently uniform. For other properties, we will need to consider how the edge distribution between the various $G_{i}$ interact with each other. In bounding the probabilities of certain events, we will often consider the probability of the event given $G_{i}$ is picked at random conditioned on the event that $G_{j}$ with $j<i$ are already chosen.

In the random graph $G(n, p)$ on $n$ vertices with each edge taken with probability $p$ independently of the other edges, the expected degree of each vertex is $p(n-1)$, and the following simple lemma shows that with high probability no vertex will have degree which deviates much from this quantity. We will assume throughout this subsection that $n \geq m_{1} \geq 2^{200}$.

Lemma 3.1 The probability that in the random graph $G(n, p)$ there is a vertex $v$ whose degree satisfies $|\operatorname{deg}(v)-p n|>n^{3 / 4}$ is at most $e^{-n^{1 / 2}}$.

Proof: For a fixed vertex $v$, its $\operatorname{degree} \operatorname{deg}(v)$ follows a binomial distribution with parameters $n-1$ and $p$. Note that if $|\operatorname{deg}(v)-p n|>n^{3 / 4}$ then also $|\operatorname{deg}(v)-p(n-1)|>n^{3 / 4}-1$. From the Chernoff-type estimate (1), we get that the probability $|\operatorname{deg}(v)-p n|>n^{3 / 4}$ is at most $2 e^{-2\left(n^{3 / 4}-1\right)^{2} /(n-1)} \leq \frac{1}{n} e^{-n^{1 / 2}}$. As there are $n$ vertices, from the union bound, we get the probability that there is a vertex $v$ with $|\operatorname{deg}(v)-p n|>n^{3 / 4}$ is at most $e^{-n^{1 / 2}}$.

For $X \in P_{i}$, we will use $N(X)$ to denote the neighborhood of $X$ in graph $G_{i}$, that is, the set of $Y \in P_{i}$ such that $(X, Y)$ is an edge of $G_{i}$. We have the following corollary of Lemma 3.1.

Corollary 3.1 Let $E_{1}$ be the event that there is $i, 1 \leq i \leq s-1$, such that $G_{i}$ has a vertex $X$ with degree $|N(X)|$ satisfying ||$N(X)\left|-p_{i} m_{i}\right|>m_{i}^{3 / 4}$. The probability of event $E_{1}$ is at most $\pi_{1}:=\sum_{i=1}^{s-1} e^{-m_{i}^{1 / 2}}$.

Lemma 3.2 Suppose $\nu=3 \sum_{i=1}^{s-1} p_{i} \leq 1 / 2$. For $2 \leq i \leq s-1$, let $E_{2 i}$ be the event that $G_{i}$ has less than $\frac{1}{4} p_{i} m_{i}^{2}$ edges which are not edges of $G^{i}$. Let $E_{2}$ be the event that none of the events $E_{2 i}$, $2 \leq i \leq s-1$, occurs. The probability $\pi_{2}$ of event $E_{2}$ is at most $\pi_{1}+\sum_{i=2}^{s-1} e^{-p_{i}^{2} m_{i}^{2} / 24}$, where $\pi_{1}$ is defined in Corollary 3.1 .

Proof: If event $E_{1}$ does not occur, given $\nu \leq 1 / 2$, then the number of edges of $G^{i}$ is at most

$$
\left(\sum_{j=1}^{i-1} p_{j}+m_{j}^{-1 / 4}\right) m_{i}^{2} / 2 \leq \frac{\nu}{4} m_{i}^{2} \leq m_{i}^{2} / 8 .
$$

Each of the remaining at least $\left(\begin{array}{c}m_{i} \\ 2\end{array}\right)-\frac{1}{8} m_{i}^{2} \geq m_{i}^{2} / 3$ unordered pairs of parts of $P_{i}$ has probabilty $p_{i}$ of being an edge of $G_{i}$, independently of each other. The expected number of edges of $G_{i}$ which are not edges of $G^{i}$ is therefore at least $\frac{p_{i} m_{i}^{2}}{3}=\frac{p_{i} m_{i}^{2}}{4}+\frac{p_{i} m_{i}^{2}}{12}$. By (11), the probability of event $E_{2 i}$ given the number of edges of $G^{i}$ is at most $m_{i}^{2} / 8$ is at most $e^{-2\left(p_{i} m_{i}^{2} / 12\right)^{2} /\left(m_{i}^{2} / 3\right)}=e^{-p_{i}^{2} m_{i}^{2} / 24}$. Summing over all $i$, the probability of event $E_{2}$ given $E_{1}$ does not occur is at most $\sum_{i=2}^{s-1} e^{-p_{i}^{2} m_{i}^{2} / 24}$. We thus have that the probability of $E_{2}$ is at most $\pi_{2}$. 
In a graph $G$ with vertex subsets $U, W$, we let $d_{G}(U, W)$ denote the fraction of pairs in $U \times W$ which are edges of $G$. If $U=\{u\}$ consists of a single vertex $u$, we let $d_{G}(u, W)=d_{G}(U, W)$. If the underlying graph $G$ is clear, we will sometimes write $d(U, W)$ for $d_{G}(U, W)$. The following lemma shows that there is a low probability that the density between a vertex and certain vertex subsets is large.

Lemma 3.3 Let $E_{3}$ be the event that there is $i, 1 \leq i \leq s-2$, and distinct $X, Y \in P_{i}, d \in\{1,2\}$, and $v \in X_{Y}^{3-d}$, such that $(X, Y)$ is an edge of $G_{i}$ but not an edge of $G^{i}$, and $d_{G}\left(v, Y_{X}^{d}\right)>\nu$. The probability of event $E_{3}$ is at most $\pi_{3}:=\sum_{i=1}^{s-2} \sum_{j=i+1}^{s-1} m_{i} m_{j} e^{-4 p_{j}^{2} m_{j} / m_{i}}$.

Proof: If $(X, Y)$ is an edge of $G_{i}$ but not an edge of $G^{i}$, then none of the edges of $G$ between $X_{Y}^{3-d}$ and $Y_{X}^{d}$ come from the edges of any $G_{j}$ with $j \leq i$. So for event $E_{3}$ to occur, there must be $1 \leq i<j \leq s-1, X, Y \in P_{i}$ with $(X, Y)$ an edge of $G_{i}$, and $X^{\prime} \in P_{j}$ with $X^{\prime} \subset X_{Y}^{3-d}$, such that $d_{G_{j}}\left(X^{\prime}, Y^{*}\right)>3 p_{j}$, where $Y^{*}$ denotes the set of $Y^{\prime} \in P_{j}$ with $Y^{\prime} \subset Y_{X}^{d}$.

Fix for now $i, 1 \leq i \leq s-2$, and $j$ with $i+1 \leq j \leq s-1$. Fix also an edge $(X, Y)$ of $G_{i}$ which is not an edge of $G^{i}$ and $d \in\{1,2\}$. Fix a set $X^{\prime} \in P_{j}$ with $X^{\prime} \subset X_{Y}^{3-d}$ and as before let $Y^{*}$ denote the set of all $Y^{\prime} \in P_{j}$ with $Y^{\prime} \subset Y_{X}^{d}$. The probability that $d_{G_{j}}\left(X^{\prime}, Y^{*}\right)>3 p_{j}$ is by (10) at most $e^{-2\left(2 p_{j}\left|Y^{*}\right|\right)^{2} /\left|Y^{*}\right|}=e^{-4 p_{j}^{2} m_{j} / m_{i}}$, since $\left|Y^{*}\right|=\frac{m_{j}}{2 m_{i}}$. Summing over all possible choices of $i, j,(X, Y), d$, and $X^{\prime} \in P_{j}$ with $X^{\prime} \subset X_{Y}^{3-d}$, by the union bound we have the probability of event $E_{3}$ is at most

$$
\sum_{i=1}^{s-2} \sum_{j=i+1}^{s-1} 2 m_{i}^{2} \cdot \frac{m_{j}}{2 m_{i}} e^{-4 p_{j}^{2} m_{j} / m_{i}}=\pi_{3} .
$$

Note that the condition that $(X, Y)$ is an edge of $G_{i}$ but not of $G^{i}$ is necessary, since it guarantees that none of the edges in $G_{j}$ with $j \leq i$ contributes to the edges between $X_{Y}^{3-d}$ and $Y_{X}^{d}$ in $G$. If $(X, Y)$ was an edge of $G^{i}$, then we would have a complete bipartite graph between $X$ and $Y$ and hence $d_{G}\left(v, Y_{X}^{d}\right)=1$.

The codegree $\operatorname{codeg}(u, v)$ of two vertices $u$ and $v$ is the number of vertices $w$ which are connected to both $u$ and $v$. A second useful fact about $G(n, p)$ is that with high probability the codegree of any two vertices $u$ and $v$ is roughly $p^{2} n$.

Lemma 3.4 The probability that in the random graph $G(n, p)$ there are distinct vertices $u$ and $v$ with $\left|\operatorname{codeg}(u, v)-p^{2} n\right|>n^{3 / 4}$ is at most $e^{-n^{1 / 2}}$.

Proof: For fixed distinct vertices $u$ and $v$, the $\operatorname{codegree} \operatorname{codeg}(u, v)$ is binomially distributed with parameters $n-2$ and $p^{2}$. Note that if $\left|\operatorname{codeg}(u, v)-p^{2} n\right|>n^{3 / 4}$ then $\left|\operatorname{codeg}(u, v)-p^{2}(n-2)\right|>$ $n^{3 / 4}-2$. By Chernoff's inequality (1), the probability that $\left|\operatorname{codeg}(u, v)-p^{2} n\right|>n^{3 / 4}$ is at most $2 e^{-2\left(n^{3 / 4}-2\right)^{2} /(n-2)} \leq n^{-2} e^{-n^{1 / 2}}$. Using the union bound over all $\left(\begin{array}{l}n \\ 2\end{array}\right)$ choices of $u$ and $v$ yields the result.

We have the following corollary of Lemma 3.4. 
Corollary 3.2 Let $E_{4}$ be the event that there is $i, 1 \leq i \leq s-1$, such that $G_{i}$ has vertices $X, Y \in P_{i}$ with codegree satisfying $\left|\operatorname{codeg}(X, Y)-p_{i}^{2} m_{i}\right|>m_{i}^{3 / 4}$. The probability of event $E_{4}$ is at most $\pi_{4}=$ $\sum_{i=1}^{s-1} e^{-m_{i}^{1 / 2}}$.

For $X \in P_{i}$, let $U(X)=\bigcup_{Y \in N(X)} Y$. The following three lemmas will be used to prove Lemma 3.8, which bounds the probability that there is $i, 1 \leq i \leq s-1, X \in P_{i}$, and a vertex $v \notin X$ such that $d_{G}(v, U(X))>\nu$. The proof, which puts together the next three lemmas, makes sure that it is unlikely that any $G_{j}$ contributes too much to the density between $v$ and $U(X)$.

Lemma 3.5 Fix $1 \leq i<s$. The probability that there is a pair of distinct sets $X, Y \in P_{i}$ which satisfy $d_{G_{i}}(Y, N(X))>2 p_{i}$ is at most $\pi_{5 i}:=2 e^{-m_{i}^{1 / 2}}$.

Proof: From Lemma 3.1, we know that $|N(X)| \geq p_{i} m_{i}-m_{i}^{3 / 4} \geq 3 p_{i} m_{i} / 4$ for all $X \in P_{i}$ with probability at least $1-e^{-m_{i}^{1 / 2}}$. Also, by Lemma 3.4 we have $\operatorname{codeg}(X, Y) \leq p_{i}^{2} m_{i}+m_{i}^{3 / 4} \leq 3 p_{i}^{2} m_{i} / 2$ for all distinct $X, Y \in P_{i}$ in graph $G_{i}$ with probability at least $1-e^{-m_{i}^{1 / 2}}$. The number of edges between $Y$ and $N(X)$ in $G_{i}$ is just the $\operatorname{codegree} \operatorname{codeg}(X, Y)$ of $X$ and $Y$ in $G_{i}$. Therefore, $d_{G_{i}}(Y, N(X))=$ $\operatorname{codeg}(X, Y) /|N(X)|$. Hence, with probability at least $1-2 e^{-m_{i}^{1 / 2}}$, we have

$$
d_{G_{i}}(Y, N(X))=\frac{\operatorname{codeg}(X, Y)}{|N(X)|} \leq \frac{3 p_{i}^{2} m_{i} / 2}{3 p_{i} m_{i} / 4}=2 p_{i} .
$$

Lemma 3.6 Fix $1<i<s$. Suppose every vertex of $G^{i}$ has degree at most $\nu_{i} m_{i} / 2$, where $\nu_{i}=$ $3 \sum_{j<i} p_{j}$. The probability that there is a pair of distinct sets $X, Y \in P_{i}$ which satisfy $d_{G^{i}}(Y, N(X))>\nu_{i}$ is at most $\pi_{5 i}^{\prime}:=e^{-m_{i}^{1 / 2}}+m_{i}^{2} e^{-\nu_{i} p_{i}^{2} m_{i} / 4}$.

Proof: Note that $G^{i}$ is determined by $G_{1}, \ldots, G_{i-1}$. We show that, conditioning on $G^{i}$ has maximum degree at most $\nu_{i} m_{i} / 2$, the random graph $G_{i}$ is such that the probability that there are distinct sets $X, Y \in P_{i}$ which satisfy $d_{G^{i}}(Y, N(X))>\nu_{i}$ is at most $e^{-m_{i}^{1 / 2}}+m_{i}^{2} e^{-\nu_{i} p_{i}^{2} m_{i} / 4}$.

Fix for now $X, Y \in P_{i}$. Let $U$ be the neighborhood of $Y$ in $G^{i}$. By assumption $|U| \leq \nu_{i} m_{i} / 2$. The expectation of $|N(X) \cap U|$ is at most $p_{i} \nu_{i} m_{i} / 2$. The probability that $|N(X) \cap U|>3 p_{i} \nu_{i} m_{i} / 4$ is by (11) at most

$$
e^{-2\left(p_{i} \nu_{i} m_{i} / 4\right)^{2} /|U|} \leq e^{-2\left(\nu_{i} p_{i} m_{i} / 4\right)^{2} /\left(\nu_{i} m_{i} / 2\right)}=e^{-\nu_{i} p_{i}^{2} m_{i} / 4}
$$

By Lemma 3.1, we know that $|N(X)| \geq 3 p_{i} m_{i} / 4$ for all $X \in P_{i}$ with probability at least $1-e^{-m_{i}^{1 / 2}}$. Therefore, using the union bound, with probability at least

$$
1-e^{-m_{i}^{1 / 2}}-m_{i}^{2} e^{-\nu_{i} p_{i}^{2} m_{i} / 4}
$$

we have

for all $X, Y \in P_{i}$.

$$
d_{G^{i}}(Y, N(X))=\frac{|N(X) \cap U|}{|N(X)|} \leq \frac{\left(3 \nu_{i} p_{i} m_{i} / 4\right)}{\left(3 p_{i} m_{i} / 4\right)}=\nu_{i}
$$


Lemma 3.7 Fix $1 \leq i<j<s$. Suppose every vertex of $G_{i}$ has degree at least $p_{i} m_{i} / 2$. Let $E$ be the event that there is a set $X \in P_{i}$ and a set $Y \in P_{j}$ with $Y \not \subset X$ with more than $2 p_{j}|N(X)| \frac{m_{j}}{m_{i}}$ neighbors $Y^{\prime}$ in $G_{j}$ with $Y^{\prime} \subset U(X)$. The probability of event $E$ is at most $\pi_{5 i j}:=m_{i} m_{j} e^{-p_{j}^{2} p_{i} m_{j}}$.

Proof: The number of $Y^{\prime} \in P_{j}$ with $Y^{\prime} \subset U(X)$ is $|N(X)| \frac{m_{j}}{m_{i}}$. The probability that a given $Y$ has at least $2 p_{j}|N(X)| \frac{m_{j}}{m_{i}}$ neighbors $Y^{\prime}$ in $G_{j}$ with $Y^{\prime} \subset U(X)$ is, by (11), at most

$$
e^{-2\left(p_{j}|N(X)| \frac{m_{j}}{m_{i}}\right)^{2} /\left(|N(X)| \frac{m_{j}}{m_{i}}\right)}=e^{-2 p_{j}^{2}|N(X)| \frac{m_{j}}{m_{i}}} .
$$

As there are at most $m_{j} m_{i}$ such pairs $X, Y$, we have by the union bound, the probability of event $E$ is at most

$$
m_{i} m_{j} e^{-2 p_{j}^{2}|N(X)| \frac{m_{j}}{m_{i}}} \leq m_{i} m_{j} e^{-p_{j}^{2} p_{i} m_{j}} .
$$

From the previous three lemmas, we get the next lemma.

Lemma 3.8 Consider the event $E_{5}$ that there is $i, 1 \leq i \leq s-1, X \in P_{i}$, and vertex $v \notin X$ with $d_{G}(v, U(X))>\nu$. The probability of event $E_{5}$ is at most $\pi_{5}:=\pi_{1}+\sum_{i=1}^{s-1} \pi_{5 i}+\sum_{i=2}^{s-1} \pi_{5 i}^{\prime}+\sum_{1 \leq i<j<s} \pi_{5 i j}$.

Proof: We look at edges in $G$ between $U(X)$ and $Y$ for $X \in P_{i}$ and $Y \in P_{j}$, distinguishing three different cases, namely $j=i, j<i$ and $j>i$. For event $E_{5}$ to occur at least one of the following events occurs:

- There is $1 \leq i<s$ and distinct sets $X, Y \in P_{i}$ with $d_{G_{i}}(Y, N(X))>3 p_{i}$.

- There is $1<i<s$ and distinct sets $X, Y \in P_{i}$ which satisfy $d_{G^{i}}(Y, N(X))>\nu_{i}=\sum_{j<i} 3 p_{j}$.

- There is $1 \leq i<j<s$ and sets $X \in P_{i}$ and $Y \in P_{j}$ with $Y \not \subset X$ with $d_{G_{j}}(Y, U(X))>3 p_{j}$.

The first case is covered by Lemma 3.5, the second by Lemma 3.6 and the third by Lemma 3.7, For Lemmas 3.6 and 3.7 to be applicable, it is enough to know also that for any $i$ and any $X \in P_{i}$, $\left|N(X)-p_{i} m_{i}\right| \leq m_{i}^{3 / 4} \leq p_{i} m_{i} / 4$. But this is just the event that $E_{1}$ does not occur. From Corollary 3.1. we know this holds with probability at least $1-\pi_{1}$.

Therefore, putting everything together, the probability of event $E_{5}$ is at most

$$
\pi_{1}+\sum_{i=1}^{s-1} \pi_{5 i}+\sum_{i=2}^{s-1} \pi_{5 i}^{\prime}+\sum_{1 \leq i<j<s} \pi_{5 i j} .
$$

Lemma 3.9 Fix $1<i \leq s-1$. Let $E_{6, i}$ be the event that there is $X \in P_{i}$ such that $X$ has more than $\nu|N(X)|$ neighbors $Y$ in $G^{i}$ with $Y \in N(X)$. Let $E_{6}$ be the event that at least one of the events $E_{6, i}$ occurs for $1<i \leq s-1$. Then, the probability of event $E_{6}$ is at most $\pi_{6}:=\pi_{1}+\sum_{i=2}^{s-1} m_{i} e^{-3 \nu^{2} p_{i} m_{i} / 8}$. 
Proof: Let us assume that event $E_{1}$ does not occur. Then every vertex in $G_{i}$ has degree at least $\frac{3}{4} p_{i} m_{i}$. Moreover, for every $X \in P_{i}$, its number of neighbors in $G^{i}$ is at most

$$
\left(\sum_{j=1}^{i-1} p_{j}+m_{j}^{-1 / 4}\right) m_{i} \leq \frac{5}{12} \nu m_{i} \leq \frac{1}{2} \nu\left(m_{i}-1\right) .
$$

Furthermore, the graphs $G_{i}$ and $G^{i}$ are still independently chosen given the degree conditions imposed on them by $E_{1}$ not occuring. Fix for now $X \in P_{i}$. Then, the expected fraction of elements of $N(X)$ which are neighbors of $X$ in $G^{i}$ is at most $\nu / 2$. Therefore, given $|N(X)|$, the probability that the number of neighbors of $X$ in $G^{i}$ is more than $\nu|N(X)|$ is, by (1) and the fact that a hypergeometric distribution is at least as concentrated as the corresponding binomial distribution, at most $e^{-2(\nu|N(X)| / 2)^{2} /|N(X)|}=e^{-\nu^{2}|N(X)| / 2}$. By the union bound and the assumption that the degree of $X$ in $G_{i}$ is at least $\frac{3}{4} p_{i} m_{i}$, the probability of event $E_{6, i}$ given that $E_{1}$ does not occur is at most $m_{i} e^{-3 \nu^{2} p_{i} m_{i} / 8}$. Therefore, adding over all $i$, we get that the probability of event $E_{6}$ is at most $\pi_{1}+\sum_{i=2}^{s-1} m_{i} e^{-3 \nu^{2} p_{i} m_{i} / 8}=\pi_{6}$, as required.

Lemma 3.10 Let $E_{7}$ be the event that there is $i, 1 \leq i \leq s-1$, and vertex subsets $U_{1}, U_{2} \subset P_{i}$ of $G_{i}$ with $\left|U_{1}\right|=u_{1},\left|U_{2}\right|=u_{2}$, and $u_{1} \leq u_{2}$ such that

$$
\left|e\left(U_{1}, U_{2}\right)-p_{i} u_{1} u_{2}\right|>5 \sqrt{h}
$$

where $h=h\left(u_{1}, u_{2}\right)=u_{1} u_{2}^{2} \ln \frac{m_{i} e}{u_{2}}$. The probability of event $E_{7}$ is at most $\pi_{7}:=\sum_{i=1}^{s-1} 2 m_{i}^{-2}$.

Proof: By Lemma 2.6, for each $i$, the probability that there are subsets $U_{1}, U_{2} \subset P_{i}$ such that (7) fails is at most $2 m_{i}^{-2}$. By the union bound, the probability of event $E_{7}$ is at most $\sum_{i=1}^{s-1} 2 m_{i}^{-2}$.

We gather the previous lemmas into one result, which shows that with positive probability the edge distribution of $G$ has certain desirable properties.

Lemma 3.11 Suppose $\nu=3 \sum_{i=1}^{s-1} p_{i} \leq 1 / 2$. With probability at least $1 / 2$, the graph $G$ has the following properties for all $i, 1 \leq i \leq s-1$.

- The degree of every vertex in graph $G_{i}$ differs from $p_{i} m_{i}$ by at most $m_{i}^{3 / 4}$ and the codegree of every pair of distinct vertices differs from $p_{i}^{2} m_{i}$ by at most $m_{i}^{3 / 4}$.

- The number of edges of $G_{i}$ not in $G^{i}$ is at least $p_{i} m_{i}^{2} / 4$.

- For all $X \in P_{i}$ and vertex $v \notin X$, we have $d_{G}(v, U(X)) \leq \nu$.

- For all distinct $X, Y \in P_{i}, d \in\{1,2\}$, and $v \in X_{Y}^{3-d}$, such that $(X, Y)$ is an edge of $G_{i}$ but $X$ and $Y$ are not adjacent in $G^{i}$, we have $d_{G}\left(v, Y_{X}^{d}\right) \leq \nu$.

- For all $X \in P_{i}, X$ has at most $\nu|N(X)|$ neighbors $Y$ in $G^{i}$ with $Y \in N(X)$. 
- For all vertex subsets $U_{1}, U_{2} \subset P_{i}$ of graph $G_{i}$ with $\left|U_{1}\right|=u_{1},\left|U_{2}\right|=u_{2}$, and $u_{1} \leq u_{2}$,

$$
\left|e\left(U_{1}, U_{2}\right)-p_{i} u_{1} u_{2}\right| \leq 5 \sqrt{h},
$$

where $h=h\left(u_{1}, u_{2}\right)=u_{1} u_{2}^{2} \ln \frac{m_{i} e}{u_{2}}$.

Proof: By Corollaries 3.1, 3.2 and Lemmas 3.2, 3.3, 3.8, 3.9, 3.10 and the union bound, the probability that at least one $E_{h}, 1 \leq h \leq 7$, occurs is at most $\sum_{h=1}^{7} \operatorname{Pr}\left[E_{h}\right] \leq \sum_{h=1}^{7} \pi_{h}$. Using the estimates $\rho=2^{-20}, m_{1} \geq 2^{200}, m_{r}=m_{r-1} a_{r-1}$ for $2 \leq r \leq s$, where $a_{r-1}=2^{\left\lfloor\rho m_{r-1}^{9 / 10}\right\rfloor}$, and $p_{i} \geq m_{i}^{-1 / 10}$ for $1 \leq r \leq s-1$, it is easy to verify that each $\pi_{h} \leq 1 / 14$ and hence the probability that none of these events occur, i.e., $G$ has the desired properties, is at least $1 / 2$.

For the rest of the proof of Theorem 3.1. we suppose that $G$ has the properties described in Lemma 3.11 .

\subsection{Regular partitions are close to being refinements}

Let $\theta=m_{1}^{-1 / 2}, \zeta=\omega=20 \theta, \beta=\frac{\zeta}{m_{1}}$, and $\gamma=1-\omega$. Suppose for contradiction that there is an equitable partition $\mathcal{Z}: V=Z_{1} \cup \ldots \cup Z_{k}$ of the vertex set of $G$ such that all but at most $\eta k^{2}$ ordered pairs $\left(Z_{j}, Z_{\ell}\right)$ of parts are $(\epsilon, \delta)$-regular, but $\mathcal{Z}$ is not a $(\beta, v)$-refinement of $P_{a}$.

The two main lemmas for the proof are Lemmas 3.14 and 3.15, which show that if $Z_{j}$ satisfies certain conditions, then there are at least $\theta^{-1} \eta k$ pairs $\left(Z_{j}, Z_{\ell}\right)$ that are not $(\epsilon, \delta)$-regular. The rest of the proof, Theorem 3.2. shows that there are at least $\theta k Z_{j}$ which satisfy the conditions of Lemmas 3.14 or 3.15. Together, we get at least $\theta^{-1} \eta k \cdot \theta k=\eta k^{2}$ ordered pairs $\left(Z_{j}, Z_{\ell}\right)$ which are not $(\epsilon, \delta)$-regular, which completes the proof.

Since $P_{1}$ is a partition into $m_{1}$ parts, then, by the pigeonhole principle, each $Z_{j}$ is $\frac{1}{m_{1}}$-contained in $P_{1}$. We call $Z_{j}$ ripe with respect to $r$ if $Z_{j}$ is $\beta$-contained in $P_{r}$ but not $(1-\beta)$-contained in $P_{r}$. That is, $Z_{j}$ is ripe if there is $X \in P_{r}$ containing a $\beta$-fraction of it but no $X \in P_{r}$ containing a (1- $\beta$ )-fraction of it. Let $\psi=2^{20} \beta$. We call $Z_{j}$ shattered with respect to $r$ if $Z_{j}$ is $(1-\beta)$-contained in $P_{r}$, but at least a $\psi$-fraction of $Z_{j}$ is contained in subsets $X \cap Z_{j}$ with $X \in P_{r+1}$ and $\left|X \cap Z_{j}\right|<\beta\left|Z_{j}\right|$. The sense here is that $Z_{j}$ is shattered by the partition $P_{r+1}$ if $Z_{j}$ is almost completely contained in some $X \in P_{r}$ but it is not well-covered by $P_{r+1}$.

We say that a subset $X \subset V(\beta, \gamma)$-supports the partition $\mathcal{Z}$ if at least a $\gamma$-fraction of the elements of $X$ are in sets $Z_{j}$ which $\beta$-overlap $X$. That is, a $\gamma$-fraction of the elements of $X$ are in sets $Z_{j}$ for which $\left|X \cap Z_{j}\right| \geq \beta\left|Z_{j}\right|$.

Lemma 3.12 Each of the $m_{1}$ sets in the partition $P_{1}\left(\beta, 1-\beta m_{1}\right)$-supports $\mathcal{Z}$.

Proof: Let $X \in P_{1}$. At most a $\beta$-fraction of $V$ is in sets of the form $X \cap Z_{j}$ with $\left|X \cap Z_{j}\right|<\beta\left|Z_{j}\right|$. Hence, as $|X|=|V| / m_{1}$, at most a $\beta m_{1}$-fraction of $X$ belongs to $Z_{i}$ which do not $\beta$-overlap $X$.

Let $S_{i}$ denote the set of $X \in P_{i}$ which $(\beta, \gamma)$-support $\mathcal{Z}$. We will let $\kappa_{i}=\frac{\left|S_{i}\right|}{\left|P_{i}\right|}$. Let $W_{i}$ denote the set of $X \in P_{i}$ for which $\left|N(X) \cap S_{i}\right| \leq \kappa_{i}|N(X)| / 4$. 
Lemma 3.13 For $1 \leq i \leq s-1$ with $\kappa_{i}>100 p_{i}^{-2} m_{i}^{-1} \ln \left(m_{i} e\right)$, we have $\left|W_{i}\right| \leq 100 p_{i}^{-2} \ln \left(\kappa_{i}^{-1} e\right)$.

Proof: In graph $G_{i}$, the number $e\left(W_{i}, S_{i}\right)$ of pairs in $W_{i} \times S_{i}$ which are edges is at most $\kappa_{i} / 4$ times the sum of the degrees of the vertices in $W_{i}$. Since, by Lemma 3.11, every vertex has degree at most $2 p_{i}\left|P_{i}\right|$ in $G_{i}$, we have $e\left(W_{i}, S_{i}\right) \leq\left|W_{i}\right| \cdot\left(\kappa_{i} / 4\right) \cdot 2 p_{i}\left|P_{i}\right|=p_{i}\left|S_{i}\right|\left|W_{i}\right| / 2$. Hence, by Lemma 3.11,

$$
p_{i}\left|S_{i}\right|\left|W_{i}\right| / 2 \leq\left|e\left(W_{i}, S_{i}\right)-p_{i}\right| W_{i}|| S_{i}|| \leq 5 \sqrt{h},
$$

where $h=u_{1} u_{2}^{2} \ln \frac{m_{i} e}{u_{2}}$, and $u_{1}=\min \left(\left|W_{i}\right|,\left|S_{i}\right|\right)$ and $u_{2}=\max \left(\left|W_{i}\right|,\left|S_{i}\right|\right)$. By squaring both sides, substituting $u_{1} u_{2}=\left|W_{i}\right|\left|S_{i}\right|$ and simplifying, we have $u_{1} \leq 100 p_{i}^{-2} \ln \frac{m_{i} e}{u_{2}}$. If $u_{1}=\left|S_{i}\right|=\kappa_{i} m_{i}$, then

$$
\kappa_{i} m_{i}=u_{1} \leq 100 p_{i}^{-2} \ln \frac{m_{i} e}{u_{2}} \leq 100 p_{i}^{-2} \ln \left(m_{i} e\right),
$$

contradicting our assumption. Hence, $u_{1}=\left|W_{i}\right|$, and $\left|W_{i}\right| \leq 100 p_{i}^{-2} \ln \left(\kappa_{i}^{-1} e\right)$.

The following simple proposition demonstrates the hereditary nature of supporting sets.

Proposition 3.1 Suppose $Y \in P_{i}$ is such that $Y(\beta, \gamma)$-supports the partition $\mathcal{Z}$. Then, for each $X \in P_{i}$ distinct from $Y$ and $d \in\{1,2\}, Y_{X}^{d}(\beta / 4,1 / 4)$-supports the partition $\mathcal{Z}$.

Proof: We will use the fact $\gamma \geq 7 / 8$. The sum of $\left|Z_{t} \cap Y_{X}^{d}\right|$ over all $Z_{t}$ which $\beta$-overlap $Y$ but do not $\beta / 4$-overlap $Y_{X}^{d}$ is at most $|Y| / 4$. Since $Y(\beta, \gamma)$-supports the partition, the sum of $\left|Z_{t} \cap Y_{X}^{d}\right|$ over all $Z_{t}$ which $\beta / 4$-overlaps $Y_{X}^{d}$ is at least

$$
\left|Y_{X}^{d}\right|-(1-\gamma)|Y|-|Y| / 4 \geq|Y| / 8=\left|Y_{X}^{d}\right| / 4
$$

Hence $Y_{X}^{d}(\beta / 4,1 / 4)$-supports the partition $\mathcal{Z}$.

The following lemma shows that if $Z_{j}$ satisfies certain conditions, then there are many (at least $\theta^{-1} \eta k$ ) $Z_{\ell}$ such that $\left(Z_{j}, Z_{\ell}\right)$ is not $(\epsilon, \delta)$-regular.

Lemma 3.14 Suppose $X \in P_{i} \backslash W_{i}, \kappa_{i}=\left|S_{i}\right| /\left|P_{i}\right| \geq 1 / 2, Z_{j}$ is shattered with respect to $i$, and $Z_{j}$ $(1-\beta)$-overlaps $X$. There are at least $\theta^{-1} \eta k$ sets $Z_{\ell} \in \mathcal{Z}$ for which $\left(Z_{j}, Z_{\ell}\right)$ is not $(\epsilon, \delta)$-regular.

Proof: Since $Z_{j}$ is shattered with respect to $i$ and $Z_{j}(1-\beta)$-overlaps $X$, then $\left|X \cap Z_{j}\right| \geq(1-\beta)\left|Z_{j}\right|$, but the sum of $\left|X^{\prime} \cap Z_{j}\right|$ over all $X^{\prime} \in P_{i+1}$ with $\left|X^{\prime} \cap Z_{j}\right|<\beta\left|Z_{j}\right|$ is at least $\psi\left|Z_{j}\right|$.

Let $Z_{j}^{\prime}=X \cap Z_{j}$, so $\left|Z_{j}^{\prime}\right| \geq(1-\beta)\left|Z_{j}\right|$. For each $X^{\prime} \in P_{i+1}$ with $X^{\prime} \subset X$ and $\left|X^{\prime} \cap Z_{j}\right|<\beta\left|Z_{j}\right|$, let $\lambda_{X^{\prime}}=\left|X^{\prime} \cap Z_{j}^{\prime}\right| /\left|Z_{j}\right|$, so each $\lambda_{X^{\prime}}<\beta$, i.e., $\beta>\|\lambda\|_{\infty}$. Also, $\|\lambda\|_{1} \geq \psi-\beta$ follows from the facts that $\left|X \cap Z_{j}\right| \geq(1-\beta)\left|Z_{j}\right|$ and the sum of $\left|X^{\prime} \cap Z_{j}\right|$ over all $X^{\prime} \in P_{i+1}$ with $\left|X^{\prime} \cap Z_{j}\right|<\beta\left|Z_{j}\right|$ is at least $\psi\left|Z_{j}\right|$. Therefore,

$$
\sigma^{2}=\left(\frac{\|\lambda\|_{2}}{\|\lambda\|_{1}}\right)^{2} \leq \frac{\|\lambda\|_{\infty}}{\|\lambda\|_{1}}<\frac{\beta}{\psi-\beta}=\frac{1}{2^{20}-1}<2^{-19}
$$


By Lemma 2.3 with $\alpha=1 / 8, \mu=2 \rho^{1 / 2}=2^{-9}, \sigma<2^{-9}$, and $\tau=1-2^{-5}$, we have that the number of $Y \in N(X)$ for which

$$
\left|Z_{j} \cap X_{Y}^{1}\right|,\left|Z_{j} \cap X_{Y}^{2}\right| \geq \alpha|| \lambda||_{1}\left|Z_{j}\right| \geq \alpha(\psi-\beta)\left|Z_{j}\right|
$$

is at least $\left(1-2^{-5}\right)|N(X)|$, where $N(X)$ is the neighborhood of $X$ in graph $G_{i}$.

By Lemma 3.11, the number of $Y \in N(X)$ which are also adjacent to $X$ in $G^{i}$ is at most $\nu|N(X)|$. Also, since $X \notin W_{i}$, we have $\left|N(X) \cap S_{i}\right| \geq \kappa_{i}|N(X)| / 4$. Therefore, the number of $Y \in S_{i}$ with $(X, Y)$ an edge of $G_{i}$ but not an edge of $G^{i}$, and (8) is satisfied is at least

$$
\left(1-2^{-5}\right)|N(X)|-\left|N(X) \backslash S_{i}\right|-\nu|N(X)|>\left(\kappa_{i} / 4-2^{-5}-\nu\right)|N(X)| \geq|N(X)| / 16 .
$$

Fix such a $Y$, and let $U_{d}=Z_{j} \cap X_{Y}^{d}$ for $d \in\{1,2\}$, so $\left|U_{1}\right|,\left|U_{2}\right| \geq \alpha(\psi-\beta)\left|Z_{j}\right|$. Since $Y \in S_{i}$, we have $Y(\beta, \gamma)$-supports $\mathcal{Z}$. By Proposition 3.1, $Y_{X}^{d}(\beta / 4,1 / 4)$-supports $\mathcal{Z}$. By Lemma 3.11, for each vertex $v \in X_{Y}^{3-d}$, we have $d\left(v, Y_{X}^{d}\right) \leq \nu$. In particular, $d\left(U_{3-d}, Y_{X}^{d}\right) \leq \nu$. Let $R^{d}$ be the union of all $Z_{\ell} \cap Y_{X}^{d}$ such that $Z_{\ell} \beta / 4$-overlaps $Y_{X}^{d}$, so $R^{d}$ is a subset of $Y_{X}^{d}$ of cardinality at least $\left|Y_{X}^{d}\right| / 4$. Hence, $d\left(U_{3-d}, R^{d}\right) \leq 4 \nu$.

For $Z_{\ell}$ which $\beta / 4$-overlaps $Y_{X}^{d}$, let $Z_{\ell}^{\prime}=R^{d} \cap Z_{\ell}$, so $\left|Z_{\ell}^{\prime}\right| \geq \beta\left|Z_{\ell}\right| / 4$. We next show that there are many $Z_{\ell}^{\prime}$ which satisfy

$$
d\left(U_{3-d}, Z_{\ell}^{\prime}\right) \leq 8 \nu
$$

Indeed, the union of the $Z_{\ell}^{\prime}$ which do not satisfy (9) has cardinality at most $\frac{1}{2}\left|R^{d}\right|$, so at least $1 / 2$ of $R^{d}$ consists of the union of $Z_{\ell}^{\prime}$ which satisfy (9). The number of $\ell$ which satisfy (9) is at least

$$
\begin{aligned}
\frac{1}{2}\left|R^{d}\right| /\left|Z_{\ell}\right| & \geq \frac{1}{2}\left(\left|Y_{X}^{d}\right| / 4\right) /\left|Z_{\ell}\right| \\
& =\frac{1}{16}|Y| /\left|Z_{\ell}\right|=\frac{1}{16} k / m_{i}
\end{aligned}
$$

where in the last equality we used $|Y|=|V| / m_{i}$ and $\left|Z_{\ell}\right|=|V| / k$.

For each $Z_{\ell}^{\prime}$ which satisfies (9), we have $d\left(U_{d}, Z_{\ell}^{\prime}\right)=1$ since $(X, Y)$ is an edge of $G_{i}$ and, therefore, the density of edges between $X_{Y}^{d}$ and $Y_{X}^{d}$ is 1 . Hence

$$
d\left(U_{d}, Z_{\ell}^{\prime}\right)-d\left(U_{3-d}, Z_{\ell}^{\prime}\right) \geq 1-8 \nu \geq \epsilon
$$

Since also $\left|U_{d}\right|,\left|U_{3-d}\right| \geq \alpha(\psi-\beta)\left|Z_{j}\right| \geq \delta\left|Z_{j}\right|$, and $\left|Z_{\ell}^{\prime}\right| \geq \frac{\beta}{4}\left|Z_{\ell}\right| \geq \delta\left|Z_{\ell}\right|$, we have in this case $\left(Z_{j}, Z_{\ell}\right)$ is not $(\epsilon, \delta)$-regular.

Since the number of such $Y$ is at least $|N(X)| / 16$, we have that the number of pairs $\left(Z_{\ell}, Y_{X}^{d}\right)$ such that $Z_{\ell} \beta / 4$-overlaps $Y_{X}^{d}$ and $\left(Z_{j}, Z_{\ell}\right)$ is not $(\epsilon, \delta)$-regular is at least $\left(\frac{1}{16} k / m_{i}\right)(|N(X)| / 16) \geq 2^{-9} p_{i} k$, where we used $|N(X)| \geq \frac{1}{2} p_{i} m_{i}$ from Lemma 3.11. As $Z_{\ell} \beta / 4$-overlaps $Y_{X}^{d}$ in each such pair, a given $Z_{\ell}$ is in at most $4 \beta^{-1}$ such pairs. Hence, the number of $Z_{\ell}$ for which $\left(Z_{j}, Z_{\ell}\right)$ is not $(\epsilon, \delta)$-regular is at least $2^{-11} \beta p_{i} k \geq \theta^{-1} \eta k$.

Like Lemma 3.14, the next lemma shows that if $Z_{j}$ satisfies certain conditions, then there are at least $\theta^{-1} \eta k Z_{\ell}$ such that $\left(Z_{j}, Z_{\ell}\right)$ is not $(\epsilon, \delta)$-regular. 
Lemma 3.15 Suppose $X \in P_{i} \backslash W_{i}, \kappa_{i} \geq 1 / 2, Z_{j}$ is ripe with respect to $i$, and $Z_{j} \beta$-overlaps $X$. Then there are at least $\theta^{-1} \eta k$ sets $Z_{\ell} \in \mathcal{Z}$ for which $\left(Z_{j}, Z_{\ell}\right)$ is not $(\epsilon, \delta)$-regular.

Proof: Since $Z_{j}$ is ripe with respect to $i,\left|X \cap Z_{j}\right|<(1-\beta)\left|Z_{j}\right|$. Therefore, letting $U^{\prime}=Z_{j} \backslash X$, we have $\left|U^{\prime}\right| \geq \beta\left|Z_{j}\right|$.

By Lemma 3.11, for each vertex $v$ of $G$ which is not in $X$, we have $d(v, U(X)) \leq \nu$. Since $X \notin W_{i}$, we have

$$
\left|N(X) \cap S_{i}\right| \geq \kappa_{i}|N(X)| / 4 \geq|N(X)| / 8 .
$$

So

$$
d\left(v, \bigcup_{Y \in N(X) \cap S_{i}} Y\right) \leq 8 \nu
$$

Fix for this paragraph $Y \in N(X) \cap S_{i}$. Since $Z_{j} \beta$-overlaps $X$, there is $d=d(j, Y) \in\{1,2\}$ such that $Z_{j} \beta / 2$-overlaps $X_{Y}^{d}$. Let $U_{Y}=Z_{j} \cap X_{Y}^{d}$, so $\left|U_{Y}\right| \geq \frac{\beta}{2}\left|Z_{j}\right|$ and $d\left(U_{Y}, Y_{X}^{d}\right)=1$. As $Y \in S_{i}$, we have $Y$ $(\beta, \gamma)$-supports $\mathcal{Z}$. By Proposition 3.1, $Y_{X}^{d}(\beta / 4,1 / 4)$-supports $\mathcal{Z}$.

For $Y \in N(X) \cap S_{i}$, let $R_{Y}$ denote the set of vertices $y$ which are in $Y_{X}^{d}$ with $d=d(j, Y)$, and $y$ is also in a $Z_{\ell}$ which $\beta / 4$-overlaps $Y_{X}^{d}$, so

$$
\left|R_{Y}\right| \geq \frac{1}{4}\left|Y_{X}^{d}\right|=\frac{1}{8}|Y|=\frac{|V|}{8 m_{i}}
$$

Let $R=\bigcup_{Y \in N(X) \cap S_{i}} R_{Y}$. We have

$$
|R| \geq\left|N(X) \cap S_{i}\right| \frac{|V|}{8 m_{i}} \geq 2^{-6}|N(X)| \frac{|V|}{m_{i}} \geq 2^{-6} \frac{p_{i} m_{i}}{2} \cdot \frac{|V|}{m_{i}}=2^{-7} p_{i}|V|,
$$

where we used (12), (10), and $|N(X)| \geq p_{i} m_{i} / 2$.

By (111) and (12), we have for $v \notin X$,

$$
d(v, R) \leq 2^{6} \nu
$$

By (14), we have $d\left(U^{\prime}, R\right) \leq 2^{6} \nu$. For $Z_{\ell}$ which $\beta / 4$-overlaps $Y_{X}^{d}$ for some $Y \in N(X) \cap S_{i}$ and $d=d(j, Y)$, let $Z_{\ell}^{Y}=Z_{\ell} \cap Y_{X}^{d}$, so $\left|Z_{\ell}^{Y}\right| \geq(\beta / 4)\left|Z_{\ell}\right|$. By definition, for each $Y \in N(X) \cap S_{i}, R_{Y}$ is the union of the sets $Z_{\ell}^{Y}$. We next show that there are many $Z_{\ell}^{Y}$ which satisfy

$$
d\left(U^{\prime}, Z_{\ell}^{Y}\right) \leq 2^{7} \nu
$$

Indeed, the union of the $Z_{\ell}^{Y}$ which do not satisfy (15) has cardinality at most $\frac{1}{2}|R|$, so at least $1 / 2$ of $R$ consists of the union of $Z_{\ell}^{Y}$ which satisfy (15). The number of pairs $(\ell, Y)$ which satisfy (15)) is at least

$$
\frac{1}{2}|R| /\left|Z_{\ell}\right| \geq \frac{1}{2} 2^{-7} p_{i}|V| /\left|Z_{\ell}\right|=2^{-8} p_{i} k
$$


where we used (13) and $\left|Z_{\ell}\right|=|V| / k$. Since for each such $\ell$, we have $Z_{\ell} \beta / 4$-overlaps $Y_{X}^{d}$, each such $\ell$ is in at most $4 \beta^{-1}$ of the pairs $(\ell, Y)$ we just counted. Hence, the number of $\ell$ for which there is $Y$ such that (15) holds is at least $2^{-10} \beta p_{i} k$.

By (15) and $d\left(U_{Y}, Z_{\ell}^{Y}\right)=1$, we have

$$
d\left(U_{Y}, Z_{\ell}^{Y}\right)-d\left(U^{\prime}, Z_{\ell}^{Y}\right) \geq 1-2^{7} \nu>\epsilon
$$

and as $\left|U_{Y}\right|,\left|U^{\prime}\right| \geq \frac{\beta}{2}\left|Z_{j}\right| \geq \delta\left|Z_{j}\right|$ and $\left|Z_{\ell}^{Y}\right| \geq \frac{\beta}{4}\left|Z_{\ell}\right| \geq \delta\left|Z_{\ell}\right|$, we have that $\left(Z_{j}, Z_{\ell}\right)$ is not $(\epsilon, \delta)$-regular for at least $2^{-10} \beta p_{i} k \geq \theta^{-1} \eta k$ values of $\ell$.

The following theorem completes the proof.

Theorem 3.2 The number of ordered pairs $\left(Z_{j}, Z_{\ell}\right)$ which are not $(\epsilon, \delta)$-regular is at least $\eta k^{2}$.

Proof: By assumption, $\mathcal{Z}$ is not a $(\beta, v)$-refinement of $P_{a}$. Hence, the number of parts $Z_{j}$ of partition $\mathcal{Z}$ which are not $(1-\beta)$-contained in $P_{a}$ is at least $v k$. Let $i_{0}$ be the minimum positive integer for which $P_{i_{0}}$ is not a $(\beta, v)$-refinement of $\mathcal{Z}$. As, by assumption, $P_{a}$ is not a $(\beta, v)$-refinement of $\mathcal{Z}$, we have $1 \leq i_{0} \leq a$.

Claim 3.1 We have $\kappa_{1}=1$ and $\kappa_{i} \geq 1 / 2$ for $i<i_{0}$.

As $\beta=\zeta / m_{1}$, by Lemma 3.12, each of the $m_{1}$ parts of partition $P_{1}(\beta, 1-\zeta)$-supports $\mathcal{Z}_{j}$. As $\zeta=\omega$ and $\gamma=1-\omega$, it follows that $S_{1}=P_{1}$ and $\kappa_{1}=\left|S_{1}\right| /\left|P_{1}\right|=1$. From the definition of $i_{0}$, for each $i<i_{0}$, $P_{i_{0}}$ is a $(\beta, v)$-refinement of $\mathcal{Z}$. Fix for this paragraph such an $i<i_{0}$. Hence at most a $(\beta+v)$-fraction of the vertices are in parts $Z_{j} \cap X$ with $X \in P_{i}$ and $Z_{j} \in \mathcal{Z}$ and $\left|Z_{j} \cap X\right|<(1-\beta)\left|Z_{j}\right|$. In particular, as $1-\beta>\beta$ and $\gamma=1-\omega$, the fraction of $X \in P_{i}$ which $\operatorname{don} \operatorname{not}(\beta, \gamma)$-support $\mathcal{Z}$ is at most $\frac{\beta+v}{\omega}$. Hence $\kappa_{i} \geq 1-\frac{\beta+v}{\omega} \geq 1 / 2$, which completes the proof of Claim 3.1 .

Consider the partition $\mathcal{Z}=\mathcal{Z}^{1} \cup \mathcal{Z}^{2} \cup \mathcal{Z}^{3} \cup \mathcal{Z}^{4} \cup \mathcal{Z}^{5} \cup \mathcal{Z}^{6}$, where $Z_{j} \in \mathcal{Z}^{h}$ if $h$ is minimum such that $Z_{j}$ satisfies property $h$ below.

1. There is $i<i_{0}$ and $X \in P_{i} \backslash W_{i}$ such that $Z_{j}$ is shattered with respect to $i$ and $(1-\beta)$-overlaps $X$ or if $Z_{j}$ is ripe with respect to $i$ and $\beta$-overlaps $X$,

2. For every $X \in P_{1}$ such that $Z_{j} \beta$-overlaps $X, X \in W_{1}$.

3. There is $i, 1<i \leq i_{0}$, and $X \in W_{i}$ such that $Z_{j} \beta$-overlaps $X$,

4. $i_{0}>1$ and $Z_{j}$ is ripe with respect to $i_{0}$, and there is $X \in W_{i_{0}}$ such that $Z_{j} \beta$-overlaps $X$.

5. $Z_{j}$ is ripe with respect to $i_{0}$, and there is $X \in P_{i_{0}} \backslash W_{i_{0}}$ such that $Z_{j} \beta$-overlaps $X$.

6. $Z_{j}$ is $(1-\beta)$-contained in $P_{i_{0}}$.

It is not immediately obvious that the above six subfamilies of $\mathcal{Z}$ form a partition of $\mathcal{Z}$, so we first show that this is indeed the case. 
Claim 3.2 The above six subfamilies form a partition of $\mathcal{Z}$.

As $Z_{j} \in \mathcal{Z}^{h}$ if and only $h$ is the minimum such that $Z_{j}$ satisfies property $h$, the subfamilies $\mathcal{Z}^{h}$, $1 \leq h \leq 6$, are pairwise disjoint. We thus need to show that each $Z_{j}$ is in at least one $\mathcal{Z}^{h}$. Suppose for contradiction that $Z_{j}$ is in none of the $\mathcal{Z}^{h}$. By property $6, Z_{j}$ is not $(1-\beta)$-contained in $P_{i_{0}}$. If $Z_{j}$ is $\beta$-contained in $P_{i_{0}}$, then $Z_{j}$ is ripe with respect to $i_{0}$, and there is $X \in P_{i_{0}}$ such that $Z_{j} \beta$-overlaps $X$. Either every such $X \in W_{i_{0}}$ or there is such an $X \notin W_{i_{0}}$, and by properties 2,4 and 5 , we must have in this case $Z_{j}$ is in a $\mathcal{Z}^{h}$ for some $h \leq 5$. So $Z_{j}$ is not $\beta$-contained in $P_{i_{0}}$, and noting that every $Z_{j}$ is $\beta$-contained in $P_{1}$, we must have $Z_{j}$ is ripe or shattered with respect to at least one $i$ with $1 \leq i<i_{0}$. In particular, there is $i<i_{0}$ and $X \in P_{i}$ such that $Z_{j}$ is shattered with respect to $i$ and $(1-\beta)$-overlaps $X$ or $Z_{j}$ is ripe with respect to $i$ and $\beta$-overlaps $X$. Since $Z_{j} \notin \mathcal{Z}^{1}$, for every such $i<i_{0}$ and $X \in P_{i}$, we must have $X \in W_{i}$. But then $Z_{j} \in \mathcal{Z}^{2}$ or $\mathcal{Z}^{3}$. Thus $Z_{j}$ is in at least one of the six subfamilies, completing the claim that that these subfamilies indeed form a partition of $\mathcal{Z}$.

As the number of parts $Z_{j}$ of partition $\mathcal{Z}$ which are not $(1-\beta)$-contained in $P_{i_{0}}$ is at least $v k$, we have

$$
\left|\mathcal{Z} \backslash \mathcal{Z}^{6}\right| \geq v k
$$

Let $w_{i}=\left|W_{i}\right| /\left|P_{i}\right|$. By Claim 3.1, $\kappa_{1}=1$ and $\kappa_{i} \geq 1 / 2$ for $i<i_{0}$. Hence, from Lemma 3.13, we have $w_{1} \leq 100 p_{1}^{-2} m_{1}^{-1} \ln (2 e) \leq m_{1}^{-1 / 2}$ and similarly $w:=\sum_{1<i<i_{0}} w_{i} \leq m_{2}^{-1 / 2}$. Here we used $p_{i} \geq m_{i}^{-1 / 10}$, $m_{1} \geq 2^{200}$, and $m_{i} \geq 2^{m_{i-1}^{1 / 2}}$.

We next bound the size of $\mathcal{Z}^{2}$. If $Z_{j} \in \mathcal{Z}^{2}$, since $Z_{j}$ does not $\beta$-overlap any $X \in P_{1} \backslash W_{1}$, and $\left|P_{1}\right|=m_{1}$, then at least a $\left(1-\beta m_{1}\right)$-fraction of $Z_{j}$ is contained in sets $X \in W_{i}$. Hence, the fraction of $Z_{j} \in \mathcal{Z}$ which satisfy $Z_{j} \in \mathcal{Z}^{2}$ is at most $\left(1-\beta m_{1}\right)^{-1} w_{1} \leq 2 m_{1}^{-1 / 2}$, i.e., $\left|\mathcal{Z}^{2}\right| \leq\left(2 m_{1}^{-1 / 2}\right) k$.

Similarly, the fraction of $Z_{j} \in \mathcal{Z}$ such that there is $1<i<i_{0}$ and $X \in W_{i}$ for which $Z_{j} \beta$-overlaps $X$ is at most $\beta^{-1} m_{2}^{-1 / 2}$. Hence, $\left|\mathcal{Z}^{3}\right| \leq \beta^{-1} m_{2}^{-1 / 2} k$.

By Lemmas 3.14 and 3.15, each $Z_{j} \in \mathcal{Z}^{1}$ is in at least $\theta^{-1} \eta k$ pairs $\left(Z_{j}, Z_{\ell}\right)$ which are not $(\epsilon, \delta)$-regular. We are thus done if $\left|\mathcal{Z}^{1}\right| \geq \theta k$. So we may suppose $\left|\mathcal{Z}^{1}\right|<\theta k$.

We next give a lower bound on $\kappa_{i_{0}}$.

Claim 3.3 We have $\kappa_{i_{0}} \geq 1 / 2$.

Note that if $i_{0}=1$, by Claim [3.1, $\kappa_{1}=1$. So we may suppose that $i_{0}>1$. In order to give a lower bound on $\kappa_{i_{0}}$, we next give an upper bound on the union of all sets $Z_{j} \cap X$ with $\left|Z_{j} \cap X\right|<\beta\left|Z_{j}\right|$ and $X \in P_{i_{0}}$. If $Z_{j}$ is not $(1-\beta)$-contained in $P_{i_{0}}$, then it must be shattered or ripe with respect to some $i$ with $i<i_{0}$, or must have at most $\psi\left|Z_{j}\right|$ vertices in parts $X \cap Z_{j}$ with $X \in P_{i_{0}}$ and $\left|X \cap Z_{j}\right|<\beta\left|Z_{j}\right|$. Each $Z_{j}$ which is shattered or ripe with respect to some $i$ with $i<i_{0}$ is in $\mathcal{Z}^{1}, \mathcal{Z}^{2}$, or $\mathcal{Z}^{3}$, and hence the number of such $Z_{j}$ is at most

$$
\left|\mathcal{Z}^{1} \cup \mathcal{Z}^{2} \cup \mathcal{Z}^{3}\right| \leq \theta k+\left(2 m_{1}^{-1 / 2}\right) k+\beta^{-1} m_{2}^{-1 / 2} k .
$$

Every set $Z_{j}$ which $(1-\beta)$-overlaps $P_{i_{0}}$ has at most a $\beta$-fraction of it contained in sets $X \cap Z_{j}$ with $\left|X \cap Z_{j}\right|<\beta\left|Z_{j}\right|$ and $X \in P_{i_{0}}$. In total, we get that the fraction of vertices which belong to one of the 
sets $X \cap Z_{j}$ with $\left|X \cap Z_{j}\right|<\beta\left|Z_{j}\right|$ and $X \in P_{i_{0}}$ is at most

$$
\theta+\left(2 m_{1}^{-1 / 2}\right)+\beta^{-1} m_{2}^{-1 / 2}+\beta+\psi .
$$

The fraction of sets in $P_{i_{0}}$ which do not $(\beta, \gamma)$-support $\mathcal{Z}$ is therefore

$$
1-\kappa_{i_{0}} \leq \omega^{-1}\left(\theta+\left(2 m_{1}^{-1 / 2}\right)+\beta^{-1} m_{2}^{-1 / 2}+\beta+\psi\right) \leq 1 / 2 .
$$

Hence, $\kappa_{i_{0}} \geq 1 / 2$, which completes Claim 3.3 .

Noting that $\kappa_{i_{0}} \geq 1 / 2$, the same argument that bounded $\left|\mathcal{Z}^{3}\right|$ also gives that

$$
\left|\mathcal{Z}^{4}\right| \leq \beta^{-1} m_{2}^{-1 / 2} k \text {. }
$$

From the bounds (16), (17), (18), we have

$$
\left|\mathcal{Z}^{5}\right| \geq\left|\mathcal{Z} \backslash \mathcal{Z}^{6}\right|-\left|\mathcal{Z}^{1} \cup \mathcal{Z}^{2} \cup \mathcal{Z}^{3}\right|-\left|\mathcal{Z}^{4}\right| \geq v k-\left(\theta k+2 m_{1}^{-1 / 2} k+\beta^{-1} m_{2}^{-1 / 2} k\right)-\beta^{-1} m_{2}^{-1 / 2} k \geq \theta k .
$$

As $\kappa_{i_{0}} \geq 1 / 2$, by Lemma 3.15, each $Z_{j} \in \mathcal{Z}^{5}$ is in at least $\theta^{-1} \eta k$ pairs $\left(Z_{j}, Z_{\ell}\right)$ which are not $(\epsilon, \delta)$ regular. Hence, the number of irregular pairs is at least

$$
\left|\mathcal{Z}^{5}\right| \theta^{-1} \eta k \geq \eta k^{2}
$$

which completes the proof.

\subsection{Proof of Theorem 1.1}

To prove Theorem 1.1, it suffices to prove the following theorem.

Corollary 3.3 Let $\epsilon=1 / 2, \delta=2^{-400}, \eta<2^{-700}, s=\left\lfloor 2^{-600} \eta^{-1}\right\rfloor$, and $k$ be at most a tower of twos of height $s$. There is a graph $G=(V, E)$ for which any equitable partition $\mathcal{Z}$ of $V$ into at most $k$ parts has at least $\eta k^{2}$ ordered pairs of parts which are not $(\epsilon, \delta)$-regular.

Proof: Let $m_{1}=2^{200}$ and $p_{i}=\max \left(m_{i}^{-1 / 10}, 2^{500} \eta\right)$ for $1 \leq i \leq s-1$ and consider the graph $G$ given with positive probability by Theorem 3.1 As $\nu=3 \sum_{i=1}^{s-1} p_{i}$, we have

$$
\nu \leq 3 \sum_{i=1}^{s-1}\left(m_{i}^{-1 / 10}+2^{500} \eta\right)=3 \cdot 2^{500} \eta(s-1)+3 \sum_{i=1}^{s-1} m_{i}^{-1 / 10} \leq 3 \cdot 2^{-100}+3 \frac{3}{2} p_{1}<6 p_{1}<2^{-10},
$$

so $1-2^{7} \nu>\epsilon$. The first inequality uses that the maximum of two nonnegative real numbers is at most their sum. The second inequality uses $s=\left\lfloor 2^{-600} \eta^{-1}\right\rfloor$ and the fact that the sum of $m_{i}^{-1 / 10}$ rapidly converges, and $p_{1}=m_{1}^{-1 / 10}=2^{-20}$.

Note that as $m_{1}=2^{200}>2^{2^{2^{2}}}$ and $m_{i} \geq 2^{m_{i-1}^{1 / 2}}$ for $i>1$, we have $\left|P_{i}\right|=m_{i}$ is greater than a tower of twos of height $i+2$ for $1 \leq i \leq s$. By Theorem 3.1 with $a=s-1$, any $(\epsilon, \delta, \eta)$-regular equitable partition of $G$ is a $(\beta, v)$-refinement of $P_{s-1}$. In particular, at least one part of $P_{s-1}$ contains at least a $(1-\beta)$-fraction of a part from $\mathcal{Z}$. As $1-\beta>1 / 2$, this implies $|\mathcal{Z}| \geq \frac{1}{2}\left|P_{s-1}\right|>k$, which completes the proof. 


\section{Lower bound for the strong regularity lemma}

In this section we prove Theorem 1.2, which gives a lower bound on the strong regularity lemma and states the following. Let $0<\epsilon<2^{-100}$ and $f: \mathbb{N} \rightarrow(0,1)$ be a decreasing function with $f(1) \leq 2^{-100} \epsilon^{6}$. Define $W_{\ell}$ recursively by $W_{1}=1, W_{\ell+1}=T\left(2^{-70} \epsilon^{5} / f\left(W_{\ell}\right)\right)$, where $T$ is the tower function defined in the introduction. Let $W=W_{t-1}$ with $t=2^{-20} \epsilon^{-1}$. Then there is a graph $G$ such that if equitable partitions $\mathcal{A}, \mathcal{B}$ of the vertex set of $G$ satisfy $q(\mathcal{B}) \leq q(\mathcal{A})+\epsilon$ and $\mathcal{B}$ is $f(|\mathcal{A}|)$-regular, then $|\mathcal{A}|,|\mathcal{B}| \geq W$. We next describe the proof of Theorem 1.2. In the first subsection, we construct the graph $G$ as a specialization of the construction in Theorem [3.1. The graph $G$ we use to prove Theorem 1.2 has vertex partitions $P_{i, j}$ with $1 \leq i \leq t$, and $1 \leq j \leq h_{i}$ satisfying $P_{i^{\prime}, j^{\prime}}$ is a refinement of $P_{i, j}$ if $i^{\prime}=i$ and $j^{\prime}>j$ or if $i^{\prime}>i$. Furthermore, as the number of parts in each successive refinement is roughly exponential in the number of parts in the previous partition, we show in the first subsection that $\left|P_{t-2, h_{t-2}-2}\right| \geq W$. The edges of $G$ are defined based on certain graphs $G_{i, j}$ on $P_{i, j}$. In Subsection 4.3 , we prove a lemma which implies that the construction has the property that

$$
q\left(P_{i, h_{i}}\right)>q\left(P_{i, h_{i}-2}\right)+2 \epsilon
$$

for each $i<t$.

Let $\mathcal{A}$ and $\mathcal{B}$ be equitable partitions of the vertex set of $G$ such that $q(\mathcal{B}) \leq q(\mathcal{A})+\epsilon$ and $\mathcal{B}$ is $f(|\mathcal{A}|)$ regular. Let $M_{1}=1$ and $M_{\ell}=\left|P_{\ell-1, h_{\ell-1}-2}\right|$ for $1<\ell \leq t-1$. Let $r$ with $1 \leq r \leq t-1$ be maximum such that $|\mathcal{A}| \geq M_{r}$. Let $P^{\prime}=P_{r, h_{r}-2}$ and $P=P_{r, h_{r}}$. In Subsection 4.1, after defining $G$, we show that it satisfies the hypothesis of Theorem 3.1, and conclude that, as $\mathcal{B}$ is an $f(|\mathcal{A}|)$-regular partition of $G$ and $f$ is a decreasing function, it must be close to being a refinement of $P$. It follows that if $|\mathcal{A}| \geq M_{t-1}=\left|P_{t-2, h_{t-2}-2}\right|>W$, then $|\mathcal{B}|>W$ as well, and we are done in this case. Thus we may assume $|\mathcal{A}|<M_{t-1}$ and hence $r \leq t-2$. In Subsection 4.4 we prove

$$
q(\mathcal{A})<q\left(P^{\prime}\right)+\frac{\epsilon}{2}
$$

This follows from a lemma that states that $q\left(P^{\prime}\right)$ is close to the maximum mean square density density over all partitions of the same number of parts as $P^{\prime}$. In Subsection 4.2, we use the result that $\mathcal{B}$ is close to being a refinement of $P$ to conclude

$$
q(P) \leq q(\mathcal{B})+\frac{\epsilon}{2}
$$

Putting the three estimates (19) (with $i=r$ and noting in this case $P_{i, h_{i}}=P, P_{i, h_{i}-2}=P^{\prime}$ ), (20), (21) together, we get that

$$
q(\mathcal{B}) \geq q(P)-\frac{\epsilon}{2}>q\left(P^{\prime}\right)+2 \epsilon-\frac{\epsilon}{2}>q(\mathcal{A})+\epsilon,
$$

contradicting the hypothesis of Theorem 1.2, and completing the proof of Theorem 1.2 ,

\subsection{Construction of $G$ and proof that $\mathcal{B}$ is an approximate refinement}

We will construct the graph $G$ as a special case of the construction in Theorem 3.1 . 
Let $t=2^{-20} \epsilon^{-1}$. We have partitions $P_{\ell, j}$ of the vertex set $V$ for $1 \leq \ell \leq t$ and $1 \leq j \leq h_{\ell}$, where $h_{\ell}$ is defined later in the paragraph and $P_{\ell, j}=P_{i}$ are the partitions used to construct $G$ in Theorem 3.1 with $i=j+\sum_{d<\ell} h_{d}$. We set $m_{\ell, j}=\left|P_{\ell, j}\right|=\left|P_{i}\right|=m_{i}$, and $p_{\ell, j}=p_{i}$. As above, let $M_{1}=1$ and $M_{\ell}=m_{\ell-1, h_{\ell-1}-2}$ for $1<\ell \leq t$. Let $\epsilon_{\ell}=f\left(M_{\ell}\right), h_{\ell}=\frac{\epsilon^{5}}{2^{70} \epsilon_{\ell}}$, and $p_{\ell, j}=\max \left(m_{\ell, j}^{-1 / 10}, 2^{30} \epsilon^{-4} \epsilon_{\ell}\right)$ for $1 \leq j \leq h_{\ell}$ with $j \neq h_{\ell}-1$, and $p_{\ell, h_{\ell}-1}=\max \left(m_{\ell, j}^{-1 / 10}, 2^{30} \epsilon^{-4} \epsilon_{\ell}, 2^{10} \epsilon\right)$.

Let $m_{1}=2^{10} \epsilon^{-2}$, so $m_{1} \geq 2^{200}$. Note that, as each $m_{\ell, j}$ is exponential in a power of $m_{\ell, j-1}$, we get that $M_{\ell}$ is at least a tower of $2 \mathrm{~s}$ of height $h_{\ell}$. That is, $M_{\ell} \geq T\left(2^{-70} \epsilon^{5} / f\left(M_{\ell-1}\right)\right)$. In particular, by induction, $M_{\ell} \geq W_{\ell}$, where $W_{\ell}$ is defined earlier in this section.

We will apply Theorem 3.1 to conclude the following corollary which states that any sufficiently regular partition of $G$ is roughly a refinement of a particular $P_{\ell, j}$. To accomplish this we need to show that the conditions of the theorem hold, which we postpone until after stating the following corollary. We fix $G$ to be a graph satisfying the properties of Lemma 3.11 so that if $G$ also satisfies the conditions stated in Theorem 3.1, then it satisfies the conclusion of Theorem 3.1 .

Corollary 4.1 Let $r \leq t-1$ be the maximum positive integer for which $|\mathcal{A}| \geq M_{r}$, so $f(|\mathcal{A}|) \leq$ $f\left(M_{r}\right)=\epsilon_{r}$, and $P=P_{r, h_{r}}$. The partition $\mathcal{B}$, which is $\epsilon_{r}$-regular, is a $(\beta, v)$-refinement of $P$ with $\beta=20 m_{1}^{-3 / 2}$ and $v=5 m_{1}^{-1 / 2}$.

Note that

$$
\begin{aligned}
\nu & =3 \sum_{i=1}^{s-1} p_{i}=\sum_{\ell=1}^{t} \sum_{j=1}^{h_{\ell}} p_{\ell, j} \leq 2^{10} \epsilon t+\sum_{\ell=1}^{t} \sum_{j=1}^{h_{\ell}}\left(m_{\ell, j}^{-1 / 10}+2^{30} \epsilon^{-4} \epsilon_{\ell}\right) \\
& \leq 2^{10} \epsilon t+\sum_{i=1}^{s-1} m_{i}^{-1 / 10}+\sum_{\ell=1}^{t} \sum_{j=1}^{h_{\ell}} 2^{30} \epsilon^{-4} \epsilon_{\ell} \leq 2^{10} \epsilon t+\sum_{i=1}^{s-1} m_{i}^{-1 / 10}+\sum_{\ell=1}^{t} 2^{-40} \epsilon \leq 2^{-9},
\end{aligned}
$$

where we used that the maximum of a set of nonnegative numbers is at most their sum, and substituted in $h_{\ell}=\frac{\epsilon^{5}}{2^{70} \epsilon_{\ell}}, m_{1}=2^{10} \epsilon^{-2} \geq 2^{200}, m_{i+1}=m_{i} a_{i} \geq m_{i} 2^{\left\lfloor 2^{-20} m_{i}^{9 / 10}\right\rfloor}$, and $t=2^{-20} \epsilon^{-1}$. We thus have $1-2^{7} \nu \geq 1 / 2 \geq \epsilon_{r}$. Notice if $\eta=\epsilon_{r}=f\left(M_{r}\right)$, then, for $1 \leq i \leq r$ and $1 \leq j \leq h_{i}$, we have

$$
p_{i, j} \geq 2^{30} \epsilon^{-4} \epsilon_{i} \geq 2^{30} \epsilon^{-4} \epsilon_{r}=2^{10} \eta m_{1}^{2},
$$

where we used $m_{1}=2^{10} \epsilon^{-2}$. Since $\beta=20 m_{1}^{-3 / 2}=20 \cdot 2^{-15} \epsilon^{3}$ and $f(1) \leq 2^{-100} \epsilon^{6}$, we have $\delta=\epsilon_{r}=$ $f\left(M_{r}\right) \leq f\left(M_{1}\right)=f(1)<\beta / 4$.

By the above estimates, the conditions of Theorem 3.1 are satisfied, and Corollary 4.1 stated above indeed holds.

Note that if $r=t-1$ in Corollary 4.1, then $|\mathcal{A}| \geq M_{t-1}=\left|P_{t-2, h_{t-2}-2}\right|>W$, and $\mathcal{B}$ is a $(\beta, v)$ refinement of $P=P_{r, h_{r}}$. As $1-\beta>1 / 2$, this implies $|\mathcal{B}| \geq \frac{1}{2}\left|P_{r, h_{r}}\right|>W$, which completes the proof of Theorem 1.2 in this case. We can therefore assume $r<t-1$.

\subsection{Approximate refinements and mean square density}

From Corollary 4.1 and the following lemma, we deduce at the end of this subsection that if $P$ is the partition in Corollary 4.1, then $q(P) \leq q(\mathcal{B})+\frac{\epsilon}{2}$. 
Lemma 4.1 Suppose $G$ is a graph, $P$ is a vertex partition, and $Q$ is an equitable partition which is a $(\beta, v)$-refinement of $P$. Then $q(P) \leq q(Q)+2 \beta+\frac{1}{2} v$.

Proof: Let $Q^{\prime}$ be the common refinement of $P$ and $Q$, so $q\left(Q^{\prime}\right) \geq q(P)$. Let $X, Y \in Q$ be such that $X, Y$ are each $(1-\beta)$-contained in $P$. Let $X=X_{1} \cup \ldots \cup X_{r}$ be the partition of $X$ consisting of parts from $Q^{\prime}$ with $\left|X_{1}\right| \geq(1-\beta)|X|$, and $Y=Y_{1} \cup \ldots \cup Y_{s}$ be the partition of $Y$ consisting of parts from $Q^{\prime}$ with $\left|Y_{1}\right| \geq(1-\beta)|Y|$. Let $p=d\left(X_{1}, Y_{1}\right)$ and $p^{\prime}=\frac{1}{1-p_{1} q_{1}} \sum d\left(X_{i}, Y_{j}\right) p_{i} q_{j}$, where $p_{i}=\frac{\left|X_{i}\right|}{|X|}, q_{j}=\frac{\left|Y_{j}\right|}{|Y|}$ and the sum is over all pairs $(i, j) \in[r] \times[s]$ except $(i, j)=(1,1)$. That is, $p^{\prime}$ is the weighted average edge density between the pairs of parts except $\left(X_{1}, Y_{1}\right)$. We have

$$
\sum_{i=1}^{r} \sum_{j=1}^{s} d^{2}\left(X_{i}, Y_{j}\right) p_{i} q_{j} \leq p^{2} p_{1} q_{1}+\sum_{(i, j) \neq(1,1)} d\left(X_{i}, Y_{j}\right) p_{i} q_{j}=p_{1} q_{1} p^{2}+p^{\prime}\left(1-p_{1} q_{1}\right)
$$

and

$$
d(X, Y)=p p_{1} q_{1}+p^{\prime}\left(1-p_{1} q_{1}\right)
$$

Let $\epsilon=1-p_{1} q_{1}$, so

$$
\begin{aligned}
\sum_{i=1}^{r} \sum_{j=1}^{s} d^{2}\left(X_{i}, Y_{j}\right) p_{i} q_{j}-d^{2}(X, Y) & \leq(1-\epsilon) p^{2}+p^{\prime} \epsilon-\left(p(1-\epsilon)+p^{\prime} \epsilon\right)^{2} \\
& =\epsilon\left((1-\epsilon) p^{2}+p^{\prime}-2 p p^{\prime}(1-\epsilon)-p^{2} \epsilon\right) \\
& \leq \epsilon\left((1-\epsilon) p^{2}+p^{\prime}-2 p p^{\prime}(1-\epsilon)\right) \\
& \leq \epsilon \\
& \leq 2 \beta .
\end{aligned}
$$

The second to last inequality is by noting the right hand side of the third to last line is linear in $p^{\prime}$ and must therefore be maximized when $p^{\prime}=0$ or 1 , and the last inequality follows from $\epsilon=1-p_{1} q_{1}$ and $p_{1}, q_{1} \geq 1-\beta$.

Now for parts $X, Y \in Q$ that are not both $(1-\beta)$-contained in $P$, again letting $X=X_{1} \cup \ldots \cup X_{r}$ and $Y=Y_{1} \cup \ldots \cup Y_{s}$ be the partitions of $X$ and $Y$ consisting of parts from $Q^{\prime}$, and letting $q$ denote the edge density between $X$ and $Y$, and $p_{i}=\frac{\left|X_{i}\right|}{|X|}, q_{j}=\frac{\left|Y_{j}\right|}{|Y|}$, we have

$$
\sum_{i=1}^{r} \sum_{j=1}^{s} d^{2}\left(X_{i}, Y_{j}\right) p_{i} q_{j}-d^{2}(X, Y) \leq q-q^{2} \leq 1 / 4
$$

Since $Q$ is a $(\beta, v)$-refinement of $P$, at most $a 2 v$-fraction of the pairs of parts from $Q$ are such that not both parts are $(1-\beta)$-contained in $P$. Putting together the estimates from the last two paragraphs, we therefore get

$$
q(P) \leq q\left(Q^{\prime}\right) \leq q(Q)+2 \beta+\frac{1}{4} \cdot 2 v
$$

Noting that $m_{1}=2^{10} \epsilon^{-2}, \beta=20 m_{1}^{-3 / 2}<\epsilon / 8$ and $v=5 m_{1}^{-1 / 2} \leq \epsilon / 4$ in Corollary 4.1, we have the following corollary of Corollary 4.1 and Lemma 4.1. 
Corollary 4.2 If $P$ is the partition in Corollary 4.1 , then $q(P) \leq q(\mathcal{B})+\frac{\epsilon}{2}$.

\subsection{Mean square densities of the defining partitions}

The next lemma shows that the mean square density of each successive partition increases by a constant factor of the edge density of each $G_{i}$.

Lemma 4.2 For each $i$, we have $q\left(P_{i+1}\right) \geq q\left(P_{i}\right)+2^{-5} p_{i}$.

Proof: The fraction of pairs $(X, Y) \in P_{i} \times P_{i}$ which are edges of $G_{i}$ and not edges of $G^{i}$ is at least $p_{i} / 4$ by the second property in Lemma 3.11, For each such pair, the equitable partitions $X=X_{Y}^{1} \cup X_{Y}^{2}$, $Y=Y_{X}^{1} \cup Y_{X}^{2}$ satisfy $d\left(X_{Y}^{d}, Y_{X}^{d}\right)=1$ and $d\left(X_{Y}^{d}, Y_{X}^{3-d}\right) \leq \nu \leq 1 / 4$ for $d=1,2$. Let $d_{1}=d\left(X_{Y}^{1}, Y_{X}^{2}\right)$ and $d_{2}=d\left(X_{Y}^{2}, Y_{X}^{1}\right)$, so

$$
\sum_{i=1}^{2} \sum_{j=1}^{2} \frac{1}{4} d^{2}\left(X_{Y}^{i}, Y_{X}^{j}\right)-d^{2}(X, Y)=\frac{1}{2}+\frac{d_{1}^{2}+d_{2}^{2}}{4}-\left(\frac{1}{2}+\frac{d_{1}+d_{2}}{4}\right)^{2} \geq \frac{1}{4}-\frac{\left(d_{1}+d_{2}\right)}{4} \geq \frac{1}{8} .
$$

As we get this density increment for at least a $p_{i} / 4$-fraction of the pairs $(X, Y) \in P_{i} \times P_{i}$, we get a total density increment of at least $\frac{1}{8} \frac{p_{i}}{4}=2^{-5} p_{i}$.

We have the following corollary, noting that $p_{r, h_{r}-1} \geq 2^{10} \epsilon$.

Corollary 4.3 For $P=P_{r, h_{r}}$ and $P^{\prime}=P_{r, h_{r}-2}$, we have

$$
q(P)=q\left(P_{r, h_{r}}\right) \geq q\left(P_{r, h_{r}-1}\right)+2^{-5} p_{r, h_{r}-1} \geq q\left(P_{r, h_{r}-1}\right)+2 \epsilon \geq q\left(P^{\prime}\right)+2 \epsilon .
$$

\subsection{Quasirandomness and mean square density}

The goal of this subsection is to show that if $\mathcal{A}$ is a vertex partition of $G$ with $|\mathcal{A}| \leq\left|P_{i}\right|$, then $q(\mathcal{A})$ is at most $q\left(P_{i}\right)+p_{i}$ plus a small error term. To accomplish this, we show that the graphs used to define $G$ are quasirandom with small error.

The study of quasirandom graphs began with the papers by Thomason [42] and Chung, Graham, and Wilson [14. They showed that a large number of interesting graph properties satisfied by random graphs are all equivalent. These properties are known as quasirandom properties, and any graph that has one of these properties (and hence all of these properties) is known as a quasirandom graph.

This development was heavily influenced by and closely related to Szemerédi's regularity lemma. Furthermore, all known proofs of Szemerédi's theorem on long arithmetic progressions in dense subsets of the integers use some notion of quasirandomness. For graphs on $n$ vertices with edge density $p$ bounded away from zero, the following two properties are quasirandom properties. The first property states that the number of 4-cycles (or, equivalently, the number of closed walks of length 4) in the graph is $p^{4} n^{4}+o\left(n^{4}\right)$. The second property states that all pairs of vertex subsets $S, T$ have edge density roughly $p$ between them, apart from $o\left(n^{2}\right)$ edges. This fact, that the number of 4 -cycles in a 
graph can control the edge distribution, is quite notable. For our purposes, we will need to show that the first property implies the second property, with reasonable error estimates. The now standard proof bounds the second largest (in absolute value) eigenvalue of the adjacency matrix of the graph, and then applies the expander mixing lemma, which bounds the edge discrepancy between subsets in terms of the subset sizes and the second largest eigenvalue.

Lemma 4.3 Suppose $G=(V, E)$ is a graph with $n$ vertices and average degree $d$, and the number of closed walks of length 4 in $G$ is at most $d^{4}+\alpha n^{4}$. For all vertex subsets $S$ and $T$,

$$
\left|e(S, T)-\frac{d|S||T|}{n}\right|<\lambda \sqrt{|S||T|},
$$

where $\lambda \leq \alpha^{1 / 4} n$.

Proof: Let $A$ be the adjacency matrix of $G$, and $\lambda_{1}, \lambda_{2}, \ldots, \lambda_{n}$ be the eigenvalues of $A$, with $\left|\lambda_{1}\right| \geq$ $\left|\lambda_{2}\right| \geq \ldots \geq\left|\lambda_{n}\right|$. Let $\lambda=\left|\lambda_{2}\right|$. It is easy to check that $\lambda_{1} \geq d$. Let $\lambda=\left|\lambda_{2}\right|$. The number of closed walks of length 4 in $G$ is equal to the trace

$$
\operatorname{Tr}\left(A^{4}\right)=\sum_{i=1}^{n} \lambda_{i}^{4} \geq \lambda_{1}^{4}+\lambda^{4} .
$$

As $\lambda_{1} \geq d$, and the number of closed walks of length 4 is at most $d^{4}+\alpha n^{4}$, we conclude $\lambda \leq\left(\alpha n^{4}\right)^{1 / 4}=$ $\alpha^{1 / 4} n$. The expander mixing lemma (see Section 2.4 of [28]) states that for all vertex subsets $S, T$, we have $\left|e(S, T)-\frac{d|S||T|}{n}\right|<\lambda \sqrt{|S||T|}$. This completes the proof.

A spanning subgraph of graph $G$ is a subgraph of $G$ on the same vertex set $V$ as $G$. We let $H_{i}$ be the spanning subgraph of $G$ where vertices $u, v \in V$ are adjacent in $H_{i}$ if and only if there is an edge $(X, Y)$ of $G_{i}$, and $j \in\{1,2\}$ with $u \in X_{Y}^{j}, v \in Y_{X}^{j}$. Note that the edge set of $G$ is precisely the union of the edge sets of the $H_{i}$, although this is likely not an edge partition. We next use Lemma 4.3 to show that the edges of $H_{i}$ are uniformly distributed. That is, the edge density in $H_{i}$ is roughly the same between large vertex subsets of $V$.

Lemma 4.4 Let $|V|=n$. For each $i$, the graph $H_{i}$ on vertex set $V$ has the property that for all vertex subsets $S$ and $T$,

$$
\left|e_{H_{i}}(S, T)-\frac{p_{i}}{2}\right| S|| T||<2 m_{i}^{-1 / 80} p_{i} n \sqrt{|S||T|} .
$$

Proof: Note that each edge $(X, Y)$ of $G_{i}$ gives rise to two complete bipartite graphs, between $X_{Y}^{j}$ and $Y_{X}^{j}$ with $j \in\{1,2\}$, in $H_{i}$. In particular, each such edge of $G_{i}$ contributes $\frac{n}{2 m_{i}}$ degree in graph $H_{i}$ to each vertex in $X$ and in $Y$.

We first give a lower bound on the average degree $d$ in $H_{i}$. From the first property in Lemma 3.11 , every vertex in $G_{i}$ has degree differing from $p_{i} m_{i}$ by at most $m_{i}^{3 / 4}$. Hence, every vertex in $H_{i}$ has degree differing from $p_{i} m_{i} \frac{n}{2 m_{i}}=p_{i} n / 2$ by at most $m_{i}^{3 / 4} \cdot \frac{n}{2 m_{i}}=\frac{1}{2} m_{i}^{-1 / 4} n$. Thus, the average degree $d$ of $H_{i}$ satisfies $\left|d-\frac{1}{2} p_{i} n\right| \leq \frac{1}{2} m_{i}^{-1 / 4} n$. 
We next give an upper bound on the number $W_{4}$ of labeled closed walks of length four in $H_{i}$. By counting over the first and third vertex of the closed walk, we have $W_{4}=\sum_{u, v}\left|N_{H_{i}}(u, v)\right|^{2}$, that is, $W_{4}$ is the sum of the squares of the codegrees over all labeled pairs of vertices of $H_{i}$. By the first part of Lemma 3.11, if $X, Y$ are distinct parts of partition $P_{i}$, then the codegree of $X$ and $Y$ in $G_{i}$ is at most $p_{i}^{2} m_{i}+m_{i}^{3 / 4}$. Hence, from Corollary 2.1, if $u$ and $v$ are in different parts in the partition $P_{i}$, then

$$
\left|N_{H_{i}}(u, v)\right| \leq\left(\frac{1}{4}+a_{i}^{-1 / 4}\right)\left(p_{i}^{2} m_{i}+m_{i}^{3 / 4}\right) \frac{n}{m_{i}}=\left(\frac{1}{4}+a_{i}^{-1 / 4}\right)\left(p_{i}^{2}+m_{i}^{-1 / 4}\right) n .
$$

For each pair of vertices $u, v$ in the same part of $P_{i}$, we have $u$ and $v$ have the same neighborhood in $H_{i}$ and in this case we use the trivial estimate $\left|N_{H_{i}}(u, v)\right| \leq n$. In total, we get

$$
\begin{aligned}
W_{4} & =\sum_{u, v}\left|N_{H_{i}}(u, v)\right|^{2} \leq m_{i}\left(m_{i}-1\right)\left(\frac{n}{m_{i}}\right)^{2} \cdot\left(\left(\frac{1}{4}+a_{i}^{-1 / 4}\right)\left(p_{i}^{2}+m_{i}^{-1 / 4}\right) n\right)^{2}+m_{i}\left(\frac{n}{m_{i}}\right)^{2} \cdot n^{2} \\
& \leq\left(1+5 m_{i}^{-1 / 20}\right) p_{i}^{4} n^{4} / 16,
\end{aligned}
$$

where we used $p_{i} \geq m_{i}^{-1 / 10}, a_{i}=2^{\left\lfloor\rho m_{i}^{9 / 10}\right\rfloor}$ with $\rho=2^{-20}$ and $m_{i} \geq m_{1} \geq 2^{200}$.

Let

$$
\begin{aligned}
\alpha & =n^{-4}\left(W_{4}-d^{4}\right) \leq n^{-4}\left(W_{4}-\left(1-4 m_{i}^{-1 / 4} p_{i}^{-1}\right) p_{i}^{4} n^{4} / 16\right) \leq\left(5 m_{i}^{-1 / 20}+4 m_{i}^{-1 / 4} p_{i}^{-1}\right) p_{i}^{4} / 16 \\
& \leq m_{i}^{-1 / 20} p_{i}^{4} .
\end{aligned}
$$

By the choice of $\alpha$, we have $W_{4}=d^{4}+\alpha n^{4}$. From Lemma 4.3, we have

$$
\left|e(S, T)-\frac{d|S||T|}{n}\right|<\alpha^{1 / 4} n \sqrt{|S||T|} .
$$

Substituting in that the average degree $d$ differs from $p_{i} n / 2$ by at most $m_{i}^{-1 / 4} n / 2$, the bounds $\alpha^{1 / 4} \leq$ $m_{i}^{-1 / 80} p_{i}, m_{i}^{-1 / 4} / 2 \leq m_{i}^{-1 / 80} p_{i}$, and $|S||T| \leq n \sqrt{|S||T|}$, and using the triangle inequality, we have the desired estimate holds on the number $e_{H_{i}}(S, T)$ of edges in $H_{i}$ between $S$ and $T$.

We next prove the following lemma which estimates the edge density of $G$ between certain vertex subsets.

Lemma 4.5 Let $X, Y \in P_{i}$ be distinct with $(X, Y)$ not an edge of $G^{i}$. If also $(X, Y)$ is not an edge of $G_{i}$ and $A \subset X, B \subset Y$, or if $(X, Y)$ is an edge of $G_{i}$ and there is $j \in\{1,2\}$ such that $A \subset X_{Y}^{j}$ and $B \subset Y_{X}^{3-j}$, then

$$
\left|d_{G}(A, B)-\left(1-\prod_{h>i}\left(1-\frac{p_{h}}{2}\right)\right)\right|<6 m_{i+1}^{-1 / 80} p_{i+1} \frac{n}{\sqrt{|A||B|}},
$$

where $n=|V|$ is the number of vertices of $G$. 
Proof: For $i^{\prime} \geq i$, let $d_{i^{\prime}}$ denote the density between $A$ and $B$ of the pairs which are edges of at least one $H_{\ell}$ with $\ell \leq i^{\prime}$. In particular, by the choice of $A$ and $B$, no edges of $H_{h}$ for $h \leq i$ go between $A$ and $B$, and hence $d_{i}=0$. Furthermore, we have $d_{i+1}=d_{H_{i+1}}(A, B)$. By Lemma 4.4, the number of edges between $A$ and $B$ in $H_{i+1}$ satisfies

$$
\left|e_{H_{i+1}}(A, B)-\frac{p_{i+1}}{2}\right| A|| B|| \leq 2 m_{i+1}^{-1 / 80} p_{i+1} n \sqrt{|A||B|}
$$

Let $t_{i}=1$ and for $i^{\prime}>i$, let $t_{i^{\prime}}=\prod_{i+1 \leq h \leq i^{\prime}}\left(1-\frac{p_{h}}{2}\right)$. We prove by induction on $i^{\prime}$ that for each $i^{\prime} \geq i+1$, we have

$$
\left|d_{i^{\prime}}-\left(1-t_{i^{\prime}}\right)\right|<q \prod_{h=i+1}^{i^{\prime}}\left(1+p_{h}\right)
$$

where

$$
q=2 m_{i+1}^{-1 / 80} p_{i+1} \frac{n}{\sqrt{|A||B|}}
$$

In the base case $i^{\prime}=i+1$, we have the desired estimate (23) from dividing (22) out by $|A \| B|$. So suppose we have established (23) for $i^{\prime}$, and we next prove it for $i^{\prime}+1$, completing the proof of (23) by induction.

Let $X^{\prime}, Y^{\prime} \in P_{i^{\prime}}$ with $X^{\prime} \subset X, Y^{\prime} \subset Y$, and $\left(X^{\prime}, Y^{\prime}\right)$ not an edge of $G^{i^{\prime}}$. If $\left(X^{\prime}, Y^{\prime}\right)$ is not an edge of $G_{i^{\prime}}$, letting $A^{\prime}=X^{\prime} \cap A$ and $B^{\prime}=Y^{\prime} \cap B$, or if $\left(X^{\prime}, Y^{\prime}\right)$ is an edge of $G_{i^{\prime}}$, and letting $j \in\{1,2\}$ and $A^{\prime}=X_{Y}^{\prime j} \cap A$ and $B^{\prime}=Y_{X}^{\prime 3-j} \cap B$, we have

$$
\left|e_{H_{i^{\prime}+1}}\left(A^{\prime}, B^{\prime}\right)-\frac{p_{i^{\prime}+1}}{2}\right| A^{\prime}|| B^{\prime}|| \leq 2 m_{i^{\prime}+1}^{-1 / 80} p_{i^{\prime}+1} n \sqrt{\left|A^{\prime}\right|\left|B^{\prime}\right|} .
$$

Each such pair $X^{\prime}, Y^{\prime}$ with $\left(X^{\prime}, Y^{\prime}\right)$ not an edge of $G_{i^{\prime}}$ gives rise to a pair $\left(A^{\prime}, B^{\prime}\right)$, and each such pair with $\left(X^{\prime}, Y^{\prime}\right)$ an edge of $G_{i^{\prime}}$ gives rise to two pairs $\left(A^{\prime}, B^{\prime}\right)$ of this form, one for each $j \in\{1,2\}$. The number of pairs $\left(X^{\prime}, Y^{\prime}\right)$ is $\left(1-d_{G^{i^{\prime}}}(X, Y)\right)\left(m_{i^{\prime}} / m_{i}\right)^{2}$. The total number $\Delta$ of such pairs $\left(A^{\prime}, B^{\prime}\right)$ is therefore

$$
\Delta=\left(1-d_{G^{i^{\prime}}}(X, Y)+d_{G_{i^{\prime}}}(X, Y)\right)\left(m_{i^{\prime}} / m_{i}\right)^{2} .
$$

On the other hand, the sum of $\left|A^{\prime}\right|\left|B^{\prime}\right|$ over all such pairs is $\left(1-d_{i^{\prime}}\right)|A||B|$. Hence, the average value of $\left|A^{\prime}\right|\left|B^{\prime}\right|$ over all such pairs $\left(A^{\prime}, B^{\prime}\right)$ is $\left(1-d_{i^{\prime}}\right)|A||B| / \Delta$.

By the triangle inequality, summing over all such pairs $A^{\prime}, B^{\prime}$, we have the number of edges $E$ of $H_{i^{\prime}+1}$ between $A$ and $B$ which are not edges of any $H_{\ell}$ with $\ell \leq i^{\prime}$ satisfies

$$
\begin{aligned}
\left|E-\frac{p_{i^{\prime}+1}}{2}\left(1-d_{i^{\prime}}\right)\right| A|| B|| & \leq \sum_{A^{\prime}, B^{\prime}} 2 m_{i^{\prime}+1}^{-1 / 80} p_{i^{\prime}+1} n \sqrt{\left|A^{\prime}\right|\left|B^{\prime}\right|} \\
& \leq 2 m_{i^{\prime}+1}^{-1 / 80} p_{i^{\prime}+1} n\left(\left(1-d_{i^{\prime}}\right)|A||B|\right)^{1 / 2} \Delta^{1 / 2} \\
& \leq 4 m_{i^{\prime}+1}^{-1 / 80} p_{i^{\prime}+1} n(|A||B|)^{1 / 2} m_{i^{\prime}} / m_{i},
\end{aligned}
$$

where we used Jensen's inequality for the concave function $f(y)=y^{1 / 2}$. 
Hence,

$$
\begin{aligned}
\left|d_{i^{\prime}+1}-\left(1-t_{i^{\prime}+1}\right)\right| & =\left|d_{i^{\prime}}(A, B)+\frac{E}{|A||B|}-\left(1-t_{i^{\prime}+1}\right)\right| \leq\left|d_{i^{\prime}}-\left(1-t_{i^{\prime}}\right)\right|+\left|\frac{E}{|A||B|}-\left(t_{i^{\prime}}-t_{i^{\prime}+1}\right)\right| \\
& =\left|d_{i^{\prime}}-\left(1-t_{i^{\prime}}\right)\right|+\left|\frac{E}{|A||B|}-\frac{p_{i^{\prime}+1}}{2} t_{i^{\prime}}\right| \\
& \leq\left(1+\frac{p_{i^{\prime}+1}}{2}\right)\left|d_{i^{\prime}}-\left(1-t_{i^{\prime}}\right)\right|+\left|\frac{E}{|A||B|}-\frac{p_{i^{\prime}+1}}{2}\left(1-d_{i^{\prime}}\right)\right| \\
& \leq\left(1+\frac{p_{i^{\prime}+1}}{2}\right)\left|d_{i^{\prime}}-\left(1-t_{i^{\prime}}\right)\right|+4 m_{i^{\prime}+1}^{-1 / 80} p_{i^{\prime}+1} n(|A||B|)^{-1 / 2} m_{i^{\prime}} / m_{i} \\
& \leq q\left(1+\frac{p_{i^{\prime}+1}}{2}\right) \prod_{h=i+1}^{i^{\prime}}\left(1+p_{h}\right)+4 m_{i^{\prime}+1}^{-1 / 80} p_{i^{\prime}+1} n(|A||B|)^{-1 / 2} m_{i^{\prime}} / m_{i} \\
& \leq q \prod_{h=i+1}^{i^{\prime}+1}\left(1+p_{h}\right),
\end{aligned}
$$

which completes the induction proof of (23).

As $\sum p_{h} \leq 1$, we have $\prod\left(1+p_{h}\right) \leq e$. From (23) with $i^{\prime}=s-1$, we get

$$
\left|d_{G}(A, B)-\left(1-\prod_{h>i}\left(1-p_{h}\right)\right)\right|=\left|d_{s-1}-\left(1-t_{s-1}\right)\right|<q \prod_{h=i+1}^{s-1}\left(1+p_{h}\right)<6 m_{i+1}^{-1 / 80} p_{i+1} \frac{n}{\sqrt{|A||B|}},
$$

which completes the proof.

The following lemma is the main result in this subsection, showing that $q(\mathcal{A})-q\left(P^{\prime}\right)$ is small, where the mean square densities are with respect to the graph $G$.

Lemma 4.6 For $P^{\prime}=P_{r, h_{r}-2}$, we have $q(\mathcal{A}) \leq q\left(P^{\prime}\right)+\frac{\epsilon}{2}$.

Proof: Consider the partition $\mathcal{A}^{\prime}$ which is the common refinement of $P^{\prime}$ and $\mathcal{A}$. The number of parts of $\mathcal{A}^{\prime}$ is at most $\left|P^{\prime}\right||\mathcal{A}| \leq\left|P^{\prime}\right|^{2}$, and each part of $P^{\prime}$ is refined into at most $|\mathcal{A}| \leq\left|P^{\prime}\right|$ parts of $\mathcal{A}^{\prime}$. Let $i$ be such that $P_{i}=P_{r, h_{r}-2}=P^{\prime}$. As $\mathcal{A}^{\prime}$ is a refinement of $P^{\prime}$, in $H_{j}$ for each $j<i$ between each pair of parts of $\mathcal{A}^{\prime}$ the edge density is 0 or 1 . Noting that $\mathcal{A}^{\prime}$ is a refinement of $\mathcal{A}$, we have

$$
q(\mathcal{A})-q\left(P_{i}\right) \leq q\left(\mathcal{A}^{\prime}\right)-q\left(P_{i}\right)=\sum_{X, Y \in P_{i}} m_{i}^{-2} \sum_{A, B \subset \mathcal{A}^{\prime}, A \subset X, B \subset Y} \frac{|A||B|}{|X||Y|}\left(d^{2}(A, B)-d^{2}(X, Y)\right) .
$$

Note that the summand in the above sum if $(X, Y)$ is an edge of $G^{i}$ is 0 as in this case $d(A, B)=$ $d(X, Y)=1$. We have $d^{2}(A, B)-d^{2}(X, Y) \leq 1$ for $(X, Y)$ an edge of $G_{i}$, and the fraction of pairs $(X, Y)$ which are edges of $G_{i}$ is at most $p_{i}+m_{i}^{-1 / 4}$.

For a pair $X, Y \in P_{i}$ with $(X, Y)$ not an edge of $G^{i}$ or $G_{i}, A, B \subset \mathcal{A}^{\prime}$ with $A \subset X$ and $B \subset Y$, we have by Lemma 4.5 and the triangle inequality that

$$
|d(A, B)-d(X, Y)| \leq 2 \cdot 6 m_{i+1}^{-1 / 80} p_{i+1} \frac{n}{\sqrt{|A||B|}} .
$$


Summing over all parts $A, B$ of $\mathcal{A}^{\prime}$ with $A \subset X$ and $B \subset Y$, we have

$$
\begin{aligned}
\sum_{A, B \subset \mathcal{A}^{\prime}, A \subset X, B \subset Y}|A||B|\left(d^{2}(A, B)-d^{2}(X, Y)\right) & \leq \sum_{A, B \subset \mathcal{A}^{\prime}, A \subset X, B \subset Y}|A||B| 2|d(A, B)-d(X, Y)| \\
& \leq \sum_{A, B \subset \mathcal{A}^{\prime}, A \subset X, B \subset Y} 24 m_{i+1}^{-1 / 80} p_{i+1} n \sqrt{|A||B|} \\
& \leq 24 m_{i+1}^{-1 / 80} p_{i+1} n^{2},
\end{aligned}
$$

where the first inequality follows from $a^{2}-b^{2}=(a+b)(a-b) \leq 2(a-b)$ for $0 \leq a, b \leq 1$, the second inequality is by (25), and the last inequality is by using the Cauchy-Schwarz inequality, noting that

$$
\sum_{A, B \subset \mathcal{A}^{\prime}, A \subset X, B \subset Y}|A||B|=|X||Y|=\left(n / m_{i}\right)^{2},
$$

and the number of pairs $A, B \subset \mathcal{A}^{\prime}$ satisfying $A \subset X, B \subset Y$ is at most $m_{i}^{2}$.

Dividing out by $|X||Y|=\left(n / m_{i}\right)^{2}$, we have,

$$
\sum_{A, B \subset \mathcal{A}^{\prime}, A \subset X, B \subset Y} \frac{|A||B|}{|X||Y|}\left(d^{2}(A, B)-d^{2}(X, Y)\right) \leq 24 m_{i+1}^{-1 / 80} p_{i+1} m_{i}^{2} .
$$

From the estimate (26), we have from (24) that

$$
q(\mathcal{A})-q\left(P^{\prime}\right) \leq p_{i}+m_{i}^{-1 / 4}+24 m_{i+1}^{-1 / 80} p_{i+1} m_{i}^{2} \leq 3 p_{i} \leq \frac{\epsilon}{2},
$$

where we used

$$
\begin{gathered}
p_{i}=\max \left(m_{i}^{-1 / 10}, 2^{30} \epsilon^{-4} \epsilon_{r}\right), \\
\epsilon_{r} \leq \epsilon_{1}=f(1)=2^{-100} \epsilon^{6}, \\
i \geq h_{1}-2=\frac{\epsilon^{5}}{2^{70} \epsilon_{1}}-2 \geq 2^{29} \epsilon^{-1}
\end{gathered}
$$

and hence $m_{i} \geq(6 / \epsilon)^{10}$. This completes the proof.

\section{Induced graph removal lemma}

The induced graph removal lemma states that for any fixed graph $H$ on $h$ vertices and $\epsilon>0$, there is $\delta=\delta(\epsilon, H)>0$ such that if a graph $G$ on $n$ vertices has at most $\delta n^{h}$ induced copies of $H$, then we can add or delete $\epsilon n^{2}$ edges of $G$ to obtain an induced $H$-free graph. The main goal of this section is to prove Theorem 1.3, which gives a bound on $\delta^{-1}$ which is a tower in $h$ of height polynomial in $\epsilon^{-1}$. We in fact prove the key corollary of the strong regularity lemma, Lemma 1.2, with a tower-type bound. This is sufficient to prove the desired tower-type bound for the induced graph removal lemma.

We first use the weak regularity lemma of Duke, Lefmann, and Rödl to find a large subset of a graph which is $\epsilon$-regular with itself. By iteratively pulling out such subsets and redistributing the set of 
leftover vertices, we obtain a partition of any vertex subset into large vertex subsets each of which is $\epsilon$-regular with itself. Then, in Subsection [5.4, we establish Lemma 1.3, the strong cylinder regularity lemma, with a tower-type bound. We show in Subsection 5.5 that the strong cylinder regularity lemma implies the key corollary of the strong regularity lemma, Lemma 1.2, with a tower-type bound. This in turn implies Theorem 1.3 ,

In this section and the next, we call a pair $(A, B)$ of vertex subsets of a graph $\epsilon$-regular if for all $A^{\prime} \subset A$ and $B^{\prime} \subset B$ with $\left|A^{\prime}\right| \geq \epsilon|A|$ and $\left|B^{\prime}\right| \geq \epsilon|B|$, we have $\left|d\left(A^{\prime}, B^{\prime}\right)-d(A, B)\right| \leq \epsilon$.

\subsection{The Duke-Lefmann-Rödl regularity lemma}

Given a $k$-partite graph $G=(V, E)$ with $k$-partition $V=V_{1} \cup \ldots \cup V_{k}$, we will consider a partition $\mathcal{K}$ of the cylinder $V_{1} \times \cdots \times V_{k}$ into cylinders $K=W_{1} \times \cdots \times W_{k}, W_{i} \subset V_{i}$ for $i=1, \ldots, k$, and we let $V_{i}(K)=W_{i}$. Recall from the introduction that a cylinder is $\epsilon$-regular if all the $\left(\begin{array}{l}k \\ 2\end{array}\right)$ pairs of subsets $\left(W_{i}, W_{j}\right), 1 \leq i<j \leq k$, are $\epsilon$-regular. The partition $\mathcal{K}$ is $\epsilon$-regular if all but an $\epsilon$-fraction of the $k$-tuples $\left(v_{1}, \ldots, v_{k}\right) \in V_{1} \times \cdots \times V_{k}$ are in $\epsilon$-regular cylinders in the partition $\mathcal{K}$.

The weak regularity lemma of Duke, Lefmann, and Rödl [15] is now as follows. Note that, like the Frieze-Kannan weak regularity lemma, it has only a single-exponential bound on the number of parts, which is much better than the tower-type bound on the number of parts in Szemerédi's regularity lemma. Duke, Lefmann, and Rödl [15] used their regularity lemma to derive a fast approximation algorithm for the number of copies of a fixed graph in a large graph.

Lemma 5.1 Let $0<\epsilon<1 / 2$ and $\beta=\beta(\epsilon)=\epsilon^{k^{2} \epsilon^{-5}}$. Suppose $G=(V, E)$ is a k-partite graph with $k$-partition $V=V_{1} \cup \ldots \cup V_{k}$. Then there exists an $\epsilon$-regular partition $\mathcal{K}$ of $V_{1} \times \cdots \times V_{k}$ into at most $\beta^{-1}$ parts such that, for each $K \in \mathcal{K}$ and $1 \leq i \leq k$, we have $\left|V_{i}(K)\right| \geq \beta\left|V_{i}\right|$.

\subsection{Finding an $\epsilon$-regular subset}

For a graph $G=(V, E)$, a vertex subset $U \subset V$ is $\epsilon$-regular if the pair $(U, U)$ is $\epsilon$-regular. The following lemma demonstrates that any graph contains a large vertex subset which is $\epsilon$-regular.

Lemma 5.2 For each $0<\epsilon<1 / 2$, let $\delta=\delta(\epsilon)=2^{-\epsilon^{-(10 / \epsilon)^{4}}}$. Every graph $G=(V, E)$ contains an $\epsilon$-regular vertex subset $U$ with $|U| \geq \delta|V|$.

Lemma 5.1 implies that each $k$-partite graph $G=(V, E)$ with $k$-partition $V=V_{1} \cup \ldots \cup V_{k}$ has a cylinder $K$ which is $\epsilon$-regular in which each part has size $\left|V_{i}(K)\right| \geq \epsilon^{k^{2} \epsilon^{-5}}\left|V_{i}\right|$. The proof can be easily modified to show that if each part of $G$ has the same size, then each part of the $\epsilon$-regular cylinder $K$ has equal size, which is at least $\epsilon^{k^{2} \epsilon^{-5}}\left|V_{i}\right|$. This implies that for any graph $G=(V, E)$, if $G$ has at least $k$ vertices, by considering any $k$ vertex disjoint subsets of equal size $\lfloor|G| / k\rfloor \geq|G| /(2 k)$, and then applying this result, we get the following lemma.

Lemma 5.3 For each $0<\epsilon<1 / 2$, any graph $G=(V, E)$ on at least $k$ vertices has an $\epsilon$-regular $k$-cylinder with parts of equal size, which is at least $\frac{1}{2 k} \epsilon^{k^{2} \epsilon^{-5}}|V|$. 
The $t$-color Ramsey number $r_{t}(s)$ is the minimum $k$ such that every $t$-coloring of the edges of the complete graph $K_{k}$ on $k$ vertices contains a monochromatic clique of order $s$. A simple pigeonhole argument (see [23]) gives $r_{t}(s) \leq t^{t s}$ for $t \geq 2$.

Lemma 5.4 For integers $s, t \geq 2$, let $k=t^{t s}$. Let $G=(V, E)$ be a graph on at least $k$ vertices, and $0<\alpha<1 / 2$. The graph $G$ contains an $\alpha$-regular s-cylinder with parts of equal size at least $N=\frac{1}{2 k} \alpha^{k^{2} \alpha^{-5}}|V|$ such that the density between each pair of parts differs by at most $1 / t$.

Proof: Note that $k=t^{t s} \geq r_{t}(s)$. By Lemma 5.3, $G$ contains an $\alpha$-regular $k$-cylinder $U_{1} \times \cdots \times U_{k}$ with parts of equal size at least $N$. Partition the unit interval $[0,1]=I_{1} \cup \ldots \cup I_{t}$ into $t$ intervals of length $1 / t$. Consider the edge-coloring of the complete graph on $k$ vertices $1, \ldots, k$ for which the color of edge $(i, j)$ is the number $a$ for which the density $d\left(U_{i}, U_{j}\right) \in I_{a}$. Since $k \geq r_{t}(s)$, there is a monochromatic clique of order $s$ in this $t$-coloring, and the corresponding parts form the desired $s$-cylinder.

Lemma 5.5 Suppose $\alpha \leq 1 / 9$ and $U_{1} \times \cdots \times U_{s}$ is an $\alpha$-regular cylinder in a graph $G=(V, E)$ with $s \geq 2 \alpha^{-1}$ parts $U_{i}$ of equal size and the densities between the pairs of distinct parts lie in an interval of length at most $\alpha$. Then the set $U=U_{1} \cup \ldots \cup U_{s}$ is $\epsilon$-regular with itself, where $\epsilon=3 \alpha^{1 / 2}$.

Proof: Let $A, B \subset U$ with $|A|,|B| \geq \epsilon|U|$ and, for $1 \leq i \leq s$, let $A_{i}=A \cap U_{i}$ and $B_{i}=B \cap U_{i}$. Suppose $d\left(U_{i}, U_{j}\right) \in[\gamma, \gamma+\alpha]$ for $1 \leq i<j \leq s$. Let $A^{1}$ be the union of all $A_{i}$ for which $\left|A_{i}\right| \geq \alpha\left|U_{i}\right|$ and $A^{2}=A \backslash A^{1}$. Similarly, let $B^{1}$ be the union of all $B_{i}$ for which $\left|B_{i}\right| \geq \alpha\left|U_{i}\right|$ and $B^{2}=B \backslash B^{1}$. We have $\left|A^{2}\right|<\alpha|U| \leq \alpha \epsilon^{-1}|A|$ and $\left|B^{2}\right|<\alpha|U| \leq \alpha \epsilon^{-1}|B|$.

Let $I_{1}$ denote the set of all pairs $(i, i)$ with $i \in[s], I_{2}$ the set of all pairs $(i, j) \in[s] \times[s]$ with $i \neq j$, $A_{i} \subset A^{1}$, and $B_{j} \subset B^{1}$, and $I_{3}=[s] \times[s] \backslash\left(I_{1} \cup I_{2}\right)$. Let $D\left(A_{i}, B_{j}\right)=\left|d\left(A_{i}, B_{j}\right)-\gamma\right| \frac{\left|A_{i}\right|\left|B_{j}\right|}{|A||B|}$. We have

$$
\sum_{(i, i) \in I_{1}} D\left(A_{i}, B_{i}\right) \leq \sum_{(i, i) \in I_{1}} \frac{\left|A_{i}\right|\left|B_{i}\right|}{|A||B|} \leq \max _{i} \frac{\left|B_{i}\right|}{|B|} \leq \max _{i} \frac{\left|U_{i}\right|}{|B|} \leq \frac{1}{s \epsilon}
$$

If $(i, j) \in I_{2}$, using the triangle inequality and $\alpha$-regularity,

$$
\left|d\left(A_{i}, B_{j}\right)-\gamma\right| \leq\left|d\left(A_{i}, B_{j}\right)-d\left(U_{i}, U_{j}\right)\right|+\left|d\left(U_{i}, U_{j}\right)-\gamma\right| \leq \alpha+\alpha=2 \alpha .
$$

Hence,

$$
\sum_{(i, j) \in I_{2}} D\left(A_{i}, B_{j}\right) \leq \sum_{(i, j) \in I_{2}} 2 \alpha \frac{\left|A_{i}\right|\left|B_{j}\right|}{|A||B|} \leq 2 \alpha
$$

Finally,

$$
\sum_{(i, j) \in I_{3}} D\left(A_{i}, B_{j}\right) \leq \sum_{(i, j) \in I_{3}} \frac{\left|A_{i}\right|\left|B_{j}\right|}{|A||B|} \leq 1-\left(1-\frac{\left|A^{2}\right|}{|A|}\right)\left(1-\frac{\left|B^{2}\right|}{|B|}\right)<1-\left(1-\alpha \epsilon^{-1}\right)^{2} \leq 2 \alpha \epsilon^{-1} .
$$


We have by the triangle inequality

$$
\begin{aligned}
|d(A, B)-\gamma| & \leq \sum_{1 \leq i, j \leq s} D\left(A_{i}, B_{j}\right) \\
& =\sum_{(i, j) \in I_{1}} D\left(A_{i}, B_{j}\right)+\sum_{(i, j) \in I_{2}} D\left(A_{i}, B_{j}\right)+\sum_{(i, j) \in I_{3}} D\left(A_{i}, B_{j}\right) \\
& \leq \frac{1}{s \epsilon}+2 \alpha+2 \alpha \epsilon^{-1} \leq \frac{\epsilon}{2} .
\end{aligned}
$$

By the triangle inequality, for any $A, B, X, Y \subset U$ each of cardinality at least $\epsilon|U|$, we have

$$
|d(A, B)-d(X, Y)| \leq|d(A, B)-\gamma|+|\gamma-d(X, Y)| \leq \frac{\epsilon}{2}+\frac{\epsilon}{2}=\epsilon .
$$

In particular, this holds for $X=Y=U$, and hence $U$ is $\epsilon$-regular.

By applying Lemma 5.4 with $\alpha=(\epsilon / 3)^{2}$ and $s=t=\left\lceil 2 \alpha^{-1}\right\rceil$, and then applying Lemma 5.5, we obtain Lemma 5.2. Note that the proof assumes that the number of vertices of the graph is sufficiently large, at least $k=t^{t s}$, but we can make this assumption as otherwise we can trivially pick $U$ to consist of a single vertex, which is $\epsilon$-regular.

The next lemma shows that if we have an $\epsilon$-regular pair, and add a small fraction of vertices to one part, then the pair is still regular, but with a slightly worse regularity.

Lemma 5.6 Suppose $A$ and $B$ are vertex subsets of a graph $G$ which form an $\epsilon$-regular pair, and $C$ is a vertex subset disjoint from $B$ of size $|C| \leq \beta|B|$. Then the pair $(A, B \cup C)$ is $\alpha$-regular with $\alpha=\epsilon+\sqrt{\beta}+\beta$.

Proof: Let $A^{\prime} \subset A$ and $B^{\prime} \cup C^{\prime} \subset B \cup C$ with $B^{\prime} \subset B, C^{\prime} \subset C,\left|A^{\prime}\right| \geq \alpha|A|$ and $\left|B^{\prime} \cup C^{\prime}\right| \geq \alpha|B \cup C|$. Note that $\left|A^{\prime}\right| \geq \alpha|A| \geq \epsilon|A|$ and $\left|B^{\prime}\right|=\left|B^{\prime} \cup C^{\prime}\right|-\left|C^{\prime}\right| \geq \alpha|B \cup C|-|C| \geq(\alpha-\beta)|B| \geq \epsilon|B|$. Since the pair $(A, B)$ is $\epsilon$-regular, we have

$$
\left|d\left(A^{\prime}, B^{\prime}\right)-d(A, B)\right| \leq \epsilon .
$$

Also, $\left|C^{\prime}\right| \leq|C| \leq \beta|B| \leq \beta|B \cup C| \leq \beta \alpha^{-1}\left|B^{\prime} \cup C^{\prime}\right|$. Therefore,

$$
\begin{aligned}
\left|d\left(A^{\prime}, B^{\prime} \cup C^{\prime}\right)-d\left(A^{\prime}, B^{\prime}\right)\right| & =\left|d\left(A^{\prime}, B^{\prime}\right) \frac{\left|B^{\prime}\right|}{\left|B^{\prime} \cup C^{\prime}\right|}+d\left(A^{\prime}, C^{\prime}\right) \frac{\left|C^{\prime}\right|}{\left|B^{\prime} \cup C^{\prime}\right|}-d\left(A^{\prime}, B^{\prime}\right)\right| \\
& =\left|d\left(A^{\prime}, C^{\prime}\right)-d\left(A^{\prime}, B^{\prime}\right)\right| \frac{\left|C^{\prime}\right|}{\left|B^{\prime} \cup C^{\prime}\right|} \leq \frac{\left|C^{\prime}\right|}{\left|B^{\prime} \cup C^{\prime}\right|} \leq \beta \alpha^{-1} .
\end{aligned}
$$

We similarly have

$$
|d(A, B \cup C)-d(A, B)|=|d(A, C)-d(A, B)| \frac{|C|}{|B \cup C|} \leq \beta .
$$

Hence, by the triangle inequality, we have $\left|d\left(A^{\prime}, B^{\prime} \cup C^{\prime}\right)-d(A, B \cup C)\right|$ is at most

$$
\begin{aligned}
\left|d\left(A^{\prime}, B^{\prime} \cup C^{\prime}\right)-d\left(A^{\prime}, B^{\prime}\right)\right|+\left|d\left(A^{\prime}, B^{\prime}\right)-d(A, B)\right|+|d(A, B)-d(A, B \cup C)| & \leq \beta \alpha^{-1}+\epsilon+\beta \\
& \leq \alpha .
\end{aligned}
$$


Hence, the pair $(A, B \cup C)$ is $\alpha$-regular.

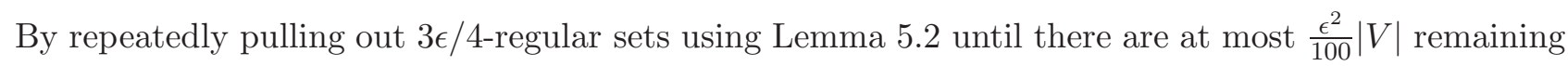
vertices, distributing the remaining vertices among the parts, and using Lemma 5.6 twice in each part, we arrive at the following lemma. It shows how to partition a graph into large parts, each part being $\epsilon$-regular with itself.

Lemma 5.7 For each $0<\epsilon<1 / 2$, let $\delta=\delta(\epsilon)=2^{-\epsilon^{-(20 / \epsilon)^{4}}}$. Every graph $G=(V, E)$ has a vertex partition $V=V_{1} \cup \ldots \cup V_{k}$ such that for each $i, 1 \leq i \leq k$, we have $\left|V_{i}\right| \geq \delta|V|$ and $V_{i}$ is an $\epsilon$-regular set.

\subsection{Tools}

In this subsection, we prove two simple lemmas concerning mean square density which will be useful in establishing and using the strong cylinder regularity lemma.

The first lemma, which is rather standard, shows that for any vertex partition $P$, there is a vertex equipartition $P^{\prime}$ with a similar number of parts to $P$ and mean square density not much smaller than the mean square density of $P$. It is useful in density increment arguments where at each stage one would like to work with an equipartition.

Lemma 5.8 Let $G=(V, E)$ be a graph, and $P: V=V_{1} \cup \ldots \cup V_{k}$ be a vertex partition into $k$ parts. There is an equitable partition $P^{\prime}$ of $V$ into $t$ parts such that $q\left(P^{\prime}\right) \geq q(P)-2 \frac{k}{t}$.

Proof: For an equipartition of $V$ into $t$ parts, we have a certain number of parts of order $\lfloor|V| / t\rfloor$ and the remaining parts are of order $\lceil|V| / t\rceil$. For each part $V_{i} \in P$, partition it into parts of order $\lfloor|V| / t\rfloor$ or $\lceil|V| / t\rceil$ so that there are not too many parts of either order to allow an equipartition of the whole set, with possibly one remaining set of cardinality less than $|V| / t$. Let $Q$ be this refinement of $P$. From the Cauchy-Schwarz inequality, it follows that $q(Q) \geq q(P)$. Let $U$ be the vertices in the remaining parts of $Q$, so $|U|<k|V| / t$.

Arbitrarily chop the vertices of $U$ into parts of the desired orders so as to obtain an equipartition $P^{\prime}$. We have

$$
q\left(P^{\prime}\right) \geq \sum_{X, Y \in Q, X, Y \subset V \backslash U} d^{2}(X, Y) \frac{|X||Y|}{|V|^{2}} \geq q(Q)-\left(1-\left(1-\frac{|U|}{|V|}\right)^{2}\right) \geq q(Q)-2 \frac{k}{t} \geq q(P)-2 \frac{k}{t} .
$$

The next lemma is helpful in deducing the induced graph removal lemma from the strong cylinder regularity lemma. Let $G=(V, E)$ and $P: V=V_{1} \cup \ldots \cup V_{k}$ be an equipartition, and $\mathcal{K}$ be a partition of the cylinder $V_{1} \times \cdots \times V_{k}$ into cylinders. For $K=W_{1} \times \cdots \times W_{k} \in \mathcal{K}$, define the density $d(K)=\frac{\left|W_{1}\right| \times \cdots \times\left|W_{k}\right|}{\left|V_{1}\right| \times \cdots \times\left|V_{k}\right|}$. The cylinder $K$ is $\epsilon$-close to $P$ if $\left|d\left(W_{i}, W_{j}\right)-d\left(V_{i}, V_{j}\right)\right| \leq \epsilon$ for all but at most $\epsilon k^{2}$ pairs $1 \leq i \neq j \leq k$. if cylinder $K$ is not $\epsilon$-close to $P$, then

$$
\sum_{1 \leq i \neq j \leq k}\left|d\left(W_{i}, W_{j}\right)-d\left(V_{i}, V_{j}\right)\right|>\epsilon^{2} k^{2}
$$


The cylinder partition $\mathcal{K}$ is $\epsilon$-close to $P$ if $\sum d(K) \leq \epsilon$, where the sum is over all $K \in \mathcal{K}$ that are not $\epsilon$-close to $P$. Note that if $\mathcal{K}$ is not $\epsilon$-close, then

$$
\sum_{K \in \mathcal{K}} \sum_{1 \leq i \neq j \leq k}\left|d\left(W_{i}, W_{j}\right)-d\left(V_{i}, V_{j}\right)\right| d(K)>\epsilon^{3} k^{2}
$$

Recall that $Q(\mathcal{K})$ is the common refinement of all the parts $V_{i}(K)$ with $i \in[k]$ and $K \in \mathcal{K}$.

Lemma 5.9 Let $G=(V, E)$ and $P: V=V_{1} \cup \ldots \cup V_{k}$ be an equipartition with no empty parts, i.e., $|V| \geq k$. Let $\mathcal{K}$ be a partition of the cylinder $V_{1} \times \cdots \times V_{k}$ into cylinders. If $Q=Q(\mathcal{K})$ satisfies $q(Q) \leq q(P)+\epsilon$, then $\mathcal{K}$ is $2^{1 / 3} \epsilon^{1 / 6}$-close to $P$.

Proof: Let $Q_{i}$ denote the partition of $V_{i}$ which is the restriction of partition $Q$ to $V_{i}$.

Since $P$ is an equipartition and $|V| \geq k$, then all parts have order at least $\left\lfloor\frac{|V|}{k}\right\rfloor \geq \frac{|V|}{2 k}$. Therefore,

$$
\epsilon \geq q(Q)-q(P)=\sum_{1 \leq i, j \leq k}\left(q\left(Q_{i}, Q_{j}\right)-q\left(V_{i}, V_{j}\right)\right) \frac{\left|V_{i}\right|\left|V_{j}\right|}{|V|^{2}} \geq \frac{1}{4 k^{2}} \sum_{1 \leq i \neq j \leq k}\left(q\left(Q_{i}, Q_{j}\right)-q\left(V_{i}, V_{j}\right)\right)
$$

where $q\left(Q_{i}, Q_{j}\right)=\sum_{A \in Q_{i}, B \in Q_{j}} d^{2}(A, B) p_{A} p_{B}$ with $p_{A}=\frac{|A|}{\left|V_{i}\right|}$ and $p_{B}=\frac{|B|}{\left|V_{j}\right|}$, and $q\left(V_{i}, V_{j}\right)=d^{2}\left(V_{i}, V_{j}\right)$. Fix for now $1 \leq i \neq j \leq k$. For $K=W_{1} \times \cdots \times W_{k} \in \mathcal{K}$, we have

$$
d\left(W_{i}, W_{j}\right)=\sum d(A, B) \frac{|A|}{\left|W_{i}\right|} \frac{|B|}{\left|W_{j}\right|},
$$

and hence, by the triangle inequality,

$$
\left|d\left(W_{i}, W_{j}\right)-d\left(V_{i}, V_{j}\right)\right| \leq \sum\left|d(A, B)-d\left(V_{i}, V_{j}\right)\right| \frac{|A|}{\left|W_{i}\right|} \frac{|B|}{\left|W_{j}\right|},
$$

where the sums are over all $A \in Q_{i}$ with $A \subset W_{i}$ and $B \in Q_{j}$ with $B \subset W_{j}$. Summing over all $K \in \mathcal{K}$, we have,

$$
\begin{aligned}
\sum_{K=W_{1} \times \cdots W_{k} \in \mathcal{K}}\left|d\left(W_{i}, W_{j}\right)-d\left(V_{i}, V_{j}\right)\right| d(K) & \leq \sum_{K=W_{1} \times \cdots W_{k} \in \mathcal{K}} \sum\left|d(A, B)-d\left(V_{i}, V_{j}\right)\right| \frac{|A|}{\left|W_{i}\right|} \frac{|B|}{\left|W_{j}\right|} d(K) \\
& =\sum_{A \in Q_{i}, B \in Q_{j}}\left|d(A, B)-d\left(V_{i}, V_{j}\right)\right| p_{A} p_{B} \\
& \leq\left(\sum_{A \in Q_{i}, B \in Q_{j}}\left(d(A, B)-d\left(V_{i}, V_{j}\right)\right)^{2} p_{A} p_{B}\right)^{1 / 2} \\
& =\left(q\left(Q_{i}, Q_{j}\right)-q\left(V_{i}, V_{j}\right)\right)^{1 / 2} .
\end{aligned}
$$

where the first equality follows by swapping the order of summation and the last inequality is the Cauchy-Schwarz inequality. 
Summing over all $1 \leq i \neq j \leq k$ and changing the order of summation,

$$
\begin{aligned}
\sum_{K=W_{1} \times \cdots W_{k} \in \mathcal{K}} \sum_{1 \leq i \neq j \leq k}\left|d\left(W_{i}, W_{j}\right)-d\left(V_{i}, V_{j}\right)\right| d(K) & \leq \sum_{1 \leq i \neq j \leq k}\left(q\left(Q_{i}, Q_{j}\right)-q\left(V_{i}, V_{j}\right)\right)^{1 / 2} \\
& \leq\left(k^{2} \sum_{1 \leq i \neq j \leq k} q\left(Q_{i}, Q_{j}\right)-q\left(V_{i}, V_{j}\right)\right)^{1 / 2} \\
& \leq \sqrt{k^{2} \cdot 4 k^{2} \epsilon}=2 \epsilon^{1 / 2} k^{2},
\end{aligned}
$$

where the second inequality is the Cauchy-Schwarz inequality and the last inequality uses the estimate (28). By the remark before the lemma, we get that $\mathcal{K}$ is $\left(2 \epsilon^{1 / 2}\right)^{1 / 3}=2^{1 / 3} \epsilon^{1 / 6}$-close to $P$.

\subsection{The strong cylinder regularity lemma}

Using the lemmas established in the previous subsections, in this subsection we prove Lemma 1.3, the strong cylinder regularity lemma, with a tower-type bound.

Recall that a $k$-cylinder $W_{1} \times \cdots \times W_{k}$ is strongly $\epsilon$-regular if all pairs $\left(W_{i}, W_{j}\right)$ with $1 \leq i, j \leq k$ are $\epsilon$-regular. A partition $\mathcal{K}$ of $V_{1} \times \cdots \times V_{k}$ into cylinders is strongly $\epsilon$-regular if all but $\epsilon\left|V_{1}\right| \times \cdots \times\left|V_{k}\right|$ vertices $\left(v_{1}, \ldots, v_{k}\right) \in V_{1} \times \cdots \times V_{k}$ are contained in strongly $\epsilon$-regular cylinders $K \in \mathcal{K}$.

We recall the statement of the strong cylinder regularity lemma.

Lemma 5.10 For $0<\epsilon<1 / 3$, positive integer $s$, and decreasing function $f: \mathbb{N} \rightarrow(0, \epsilon]$, there is $S=$ $S(\epsilon, s, f)$ such that the following holds. For every graph $G$, there is an integer $s \leq k \leq S$, an equitable partition $P: V=V_{1} \cup \ldots \cup V_{k}$ and a strongly $f(k)$-regular partition $\mathcal{K}$ of the cylinder $V_{1} \times \cdots \times V_{k}$ into cylinders satisfying that the partition $Q=Q(\mathcal{K})$ of $V$ has at most $S$ parts and $q(Q) \leq q(P)+\epsilon$. Furthermore, there is an absolute constant $c$ such that letting $s_{1}=s$ and $s_{i+1}=t_{4}\left(\left(s_{i} / f\left(s_{i}\right)\right)^{c}\right)$, we may take $S=s_{\ell}$ with $\ell=2 \epsilon^{-1}+1$.

Proof: We may assume $|V| \geq S$, as otherwise we can let $P$ and $Q$ be the trivial partitions into singletons, and it is easy to see the lemma holds. We will define a sequence of partitions $P_{1}, P_{2}, \ldots$ of equitable partitions, with $P_{j+1}$ a refinement of $P_{j}$ and $q\left(P_{j+1}\right)>q\left(P_{j}\right)+\epsilon / 2$. Let $P_{1}$ be an arbitrary equitable partition of $V$ consisting of $s_{1}=s$ parts. Suppose we have already found an equitable partition $P_{j}: V=V_{1} \cup \ldots \cup V_{k}$ with $k \leq s_{j}$.

Let $\beta(x, \ell)=x^{\ell^{2} x^{-5}}$ as in Lemma 5.1 and $\delta(x)=2^{-x^{-(20 / x)^{4}}}$ as in Lemma 5.7. We apply Lemma 5.7 to each part $V_{i}$ of the partition $P_{j}$ to get a partition of each part $V_{i}=V_{i 1} \cup \ldots \cup V_{i h_{i}}$ of $P_{i}$ into parts each of cardinality at least $\delta\left|V_{i}\right|$, where $\delta=\delta(\gamma)$ and $\gamma=f(k) \cdot \beta$ with $\beta=\beta(f(k), k)$, such that each part $V_{i h}$ is $\gamma$-regular. Note that $\delta^{-1}$ is at most triple-exponential in a polynomial in $k / f(k)$. For each $k$-tuple $\ell=\left(\ell_{1}, \ldots, \ell_{k}\right) \in\left[h_{1}\right] \times \cdots \times\left[h_{k}\right]$, by Lemma 5.1 there is an $f(k)$-regular partition $\mathcal{K}_{\ell}$ of the cylinder $V_{1 \ell_{1}} \times \cdots \times V_{k \ell_{k}}$ into at most $\beta^{-1}$ cylinders such that, for each $K \in \mathcal{K}_{\ell},\left|V_{i \ell_{i}}(K)\right| \geq \beta\left|V_{i \ell_{i}}\right|$. The union of the $\mathcal{K}_{\ell}$ forms a partition $\mathcal{K}$ of $V_{1} \times \cdots \times V_{k}$ which is strongly $f(k)$-regular. 
Recall that $Q=Q(\mathcal{K})$ is the partition of $V$ which is the common refinement of all parts $V_{i}(K)$ with $i \in[k]$ and $K \in \mathcal{K}$. The number of parts of $\mathcal{K}$ is at most $\delta^{-k} \beta^{-1}$, and hence the number of parts of $Q$ is at most $k 2^{1 /\left(\delta^{k} \beta\right)}$. Thus, the number of parts of $Q$ is at most quadruple-exponential in a polynomial in $k / f(k)$. Let $P_{j+1}$ be an equitable partition into $4 \epsilon^{-1}|Q|$ parts with $q\left(P_{j+1}\right) \geq q(Q)-\frac{\epsilon}{2}$, which exists by Lemma 5.8. Hence, there is an absolute constant $c$ such that

$$
\left|P_{j+1}\right| \leq t_{4}\left((k / f(k))^{c}\right) \leq s_{j+1}
$$

If $q(Q) \leq q\left(P_{j}\right)+\epsilon$, then we may take $P=P_{j}$ and $Q=Q(\mathcal{K})$, and these partitions satisfy the desired properties. Otherwise, $q\left(P_{j+1}\right) \geq q(Q)-\frac{\epsilon}{2}>q\left(P_{j}\right)+\frac{\epsilon}{2}$, and we continue the sequence of partitions. Since $q\left(P_{1}\right) \geq 0$, and the mean square density goes up by more than $\epsilon / 2$ at each step and is always at most 1 , this process must stop within $2 / \epsilon$ steps, and we obtain the desired partitions.

\subsection{A tower-type bound for the key corollary}

In the previous subsection, we established the strong cylinder regularity lemma with a tower-type bound. We next use this result to deduce a tower-type bound for Lemma 1.2, which is the key corollary of the strong regularity lemma, and easily implies the induced graph removal lemma as shown below. We recall the statement of Lemma 1.2 below.

Lemma 5.11 For each $0<\epsilon<1 / 3$ and decreasing function $f: \mathbb{N} \rightarrow(0, \epsilon]$ there is $\delta^{\prime}=\delta^{\prime}(\epsilon, f)$ such that every graph $G=(V, E)$ with $|V| \geq \delta^{-1}$ has an equitable partition $V=V_{1} \cup \ldots \cup V_{k}$ and vertex subsets $W_{i} \subset V_{i}$ such that $\left|W_{i}\right| \geq \delta^{\prime}|V|$, each pair $\left(W_{i}, W_{j}\right)$ with $1 \leq i \leq j \leq k$ is $f(k)$-regular, and all but at most $\epsilon k^{2}$ pairs $1 \leq i \leq j \leq k$ satisfy $\left|d\left(V_{i}, V_{j}\right)-d\left(W_{i}, W_{j}\right)\right| \leq \epsilon$. Furthermore, we may take $\delta^{\prime}=\frac{1}{8 S^{2}}$, where $S=\left(\frac{\epsilon^{6}}{4}, s, f\right)$ is defined as in Lemma 5.10 and $s=2 \epsilon^{-1}$.

Proof: Let $\alpha=\frac{\epsilon^{6}}{4}, s=2 \epsilon^{-1}$, and $\delta^{\prime}=\frac{1}{8 S^{2}}$, where $S=S(\alpha, s, f)$ is as in Lemma 5.10. We apply Lemma 5.10 with $\alpha$ in place of $\epsilon$. We get an equipartition $P: V=V_{1} \cup \ldots \cup V_{k}$ with $s \leq k \leq S$ and a strongly $f(k)$-regular partition $\mathcal{K}$ of $V_{1} \times \cdots \times V_{k}$ into cylinders such that the refinement $Q=Q(\mathcal{K})$ of $P$ has at most $S=S(\alpha, s, f)$ parts and satisfies $q(Q) \leq q(P)+\alpha$. Since $|V| \geq \delta^{\prime-1}=8 S^{2}$, and $P$ is an equipartition into $k \leq S$ parts, the cardinality of each part $V_{i} \in P$ satisfies $\left|V_{i}\right| \geq \frac{|V|}{2 S}$. By Lemma 5.9, as $2^{1 / 3} \alpha^{1 / 6}=\epsilon$, the cylinder partition $\mathcal{K}$ is $\epsilon$-close to $P$. Hence, at most an $\epsilon$-fraction of the $k$-tuples $\left(v_{1}, \ldots, v_{k}\right) \in V_{1} \times \cdots \times V_{k}$ belong to parts $K=W_{1} \times \cdots \times W_{k}$ of $\mathcal{K}$ that are not $\epsilon$-close to $P$. Since $Q(\mathcal{K})$ has at most $S$ parts, the fraction of $k$-tuples $\left(v_{1}, \ldots, v_{k}\right) \in V_{1} \times \cdots \times V_{k}$ that belong to parts $K=W_{1} \times \cdots \times W_{k}$ of $\mathcal{K}$ with $\left|W_{i}\right|<\frac{1}{4 S}\left|V_{i}\right|$ for at least one $i \in[k]$ is at most $\frac{1}{4 S} \cdot S=\frac{1}{4}$. Therefore, at least a fraction $1-f(k)-\epsilon-\frac{1}{4}>0$ of the $k$-tuples $\left(v_{1}, \ldots, v_{k}\right) \in V_{1} \times \cdots \times V_{k}$ belong to parts $K=W_{1} \times \cdots \times W_{k}$ of $\mathcal{K}$ satisfying $K$ is strongly $f(k)$-regular, $\left|W_{i}\right| \geq \frac{1}{4 S}\left|V_{i}\right| \geq \delta^{\prime}|V|$ for $i \in[k]$, and $K$ is $\epsilon$-close to $P$. Since a positive fraction of the $k$-tuples belong to such $K$, there is at least one such $K$. This $K$ has the desired properties. Indeed the number of pairs $1 \leq i \neq j \leq k$ for which $\left|d\left(W_{i}, W_{j}\right)-d\left(V_{i}, V_{j}\right)\right|>\epsilon$ is at most $\epsilon k^{2}$ and hence the number of pairs $1 \leq i \leq j \leq k$ for which $\left|d\left(W_{i}, W_{j}\right)-d\left(V_{i}, V_{j}\right)\right|>\epsilon$ is at most $\epsilon k^{2} / 2+k \leq \epsilon k^{2}$. This completes the proof. 
We next discuss how to obtain the induced graph removal lemma from Lemma 1.2. This is a bit easier to obtain than in [5] because Lemma 1.2 has the additional property that the subsets $W_{i}$ in the cylinder $K$ are $\epsilon$-regular. We finish this section by giving this proof and discussing the bound it gives for the induced graph removal lemma, which is a tower in $h$ of height polynomial in $\epsilon^{-1}$. We first need the following counting lemma, which is rather standard (see, e.g., Lemma 3.2 in Alon, Fischer, Krivelevich, and Szegedy [5] for a minor variant). We omit the proof.

Lemma 5.12 If $H$ is a graph with vertices $1, \ldots, h$, and $G$ is a graph with not necessarily disjoint vertex subsets $W_{1}, \ldots, W_{h}$ such that every pair $\left(W_{i}, W_{j}\right)$ with $1 \leq i<j \leq h$ is $\gamma$-regular with $\gamma \leq \frac{1}{4 h} \eta^{h}$, $\left|W_{i}\right| \geq \gamma^{-1}$ for $1 \leq i \leq h$ and, for $1 \leq i<j \leq k, d\left(W_{i}, W_{j}\right)>\eta$ if $(i, j)$ is an edge of $H$ and $d\left(W_{i}, W_{j}\right)<1-\eta$ otherwise, then $G$ contains at least $\left(\frac{\eta}{4}\right)^{\left(\begin{array}{c}h \\ 2\end{array}\right)}\left|W_{1}\right| \times \cdots \times\left|W_{h}\right|$ induced copies of $H$ with the copy of vertex $i$ in $W_{i}$.

We finish the section with a quantitative version of Theorem 1.3 .

Theorem 5.1 There is an absolute constant $c$ such that for any graph $H$ on $h$ vertices $1, \ldots, h$ and $0<\epsilon<1 / 2$ there is $\delta>0$ with $\delta^{-1}=t_{j}(h)$ with $j=O\left(\epsilon^{-6}\right)$ such that if a graph $G$ on $n$ vertices has at most $\delta n^{h}$ induced copies of $H$, then we can add or delete $\epsilon n^{2}$ edges of $G$ to obtain an induced $H$-free graph.

Proof: Let $\eta=\frac{\epsilon}{8}$. Let $\delta=\left(\frac{\eta}{4}\right)^{h^{2}} \delta^{\prime h}$, where $\delta^{\prime}=\delta^{\prime}(\eta, f)$ as in Lemma 5.11 and $f(k)=\frac{1}{4 h} \eta^{h}$. If the number of vertices satisfies $|V|<\delta^{-1 / h}$, then $\delta|V|^{h}<1$ and there are no induced copies of $H$, in which case no edges of $G$ need to be modified. We can therefore assume that $|V| \geq \delta^{-1 / h}=\left(\frac{\eta}{4}\right)^{-h} \delta^{\prime-1}$. As $|V| \geq \delta^{\prime-1}$, we can apply Lemma 5.11 to graph $G=(V, E)$ with $\eta$ in place of $\epsilon$ and $f$ as above. We obtain an equitable partition $V=V_{1} \cup \ldots \cup V_{k}$ and vertex subsets $W_{i} \subset V_{i}$ such that $\left|W_{i}\right| \geq \delta^{\prime}|V| \geq$ $\left(\frac{\eta}{4}\right)^{-h}$, the cylinder $W_{1} \times \cdots W_{k}$ is strongly $f(k)$-regular, and all but at most $\eta k^{2}$ pairs $1 \leq i \leq j \leq k$ satisfy $\left|d\left(W_{i}, W_{j}\right)-d\left(V_{i}, V_{j}\right)\right| \leq \eta$.

The above counting lemma shows that if there is any mapping $\phi:[h] \rightarrow[k]$ such that $d\left(W_{\phi(i)}, W_{\phi(j)}\right)>$ $\eta$ for $(i, j)$ an edge of $H$ and $d\left(W_{\phi(i)}, W_{\phi(j)}\right)<1-\eta$ for $i, j$ distinct and nonadjacent in $H$, then $G$ contains at least $\left(\frac{\eta}{4}\right)^{\left(\begin{array}{c}h \\ 2\end{array}\right)}\left|W_{1}\right| \times \cdots \times\left|W_{h}\right| \geq \delta n^{h}$ induced copies of $H$. Hence, no such mapping $\phi$ exists. That is, for every mapping $\phi:[h] \rightarrow[k]$, there is an edge $(i, j)$ for which $d\left(W_{\phi(i)}, W_{\phi(j)}\right) \leq \eta$ or distinct $i, j$ that are nonadjacent in $H$ with $d\left(W_{\phi(i)}, W_{\phi(j)}\right) \geq 1-\eta$.

For each pair $\left(W_{i}, W_{j}\right)$ for which $d\left(W_{i}, W_{j}\right) \leq \eta$, delete all edges between $V_{i}$ and $V_{j}$, and for each pair $\left(W_{i}, W_{j}\right)$ for which $d\left(W_{i}, W_{j}\right) \geq 1-\eta$, add all edges between $V_{i}$ and $V_{j}$. Let $G^{\prime}$ be this modification of $G$. By the previous paragraph, there are no induced copies of $H$ in $G^{\prime}$.

We have left to show that few edge modifications are made in obtaining $G^{\prime}$ from $G$. If a pair $\left(W_{i}, W_{j}\right)$ for which edges were modified satisfies $\left|d\left(W_{i}, W_{j}\right)-d\left(V_{i}, V_{j}\right)\right| \leq \eta$, then the density between the two sets was only changed by at most $2 \eta$. The number of $1 \leq i \leq j \leq k$ for which $\left|d\left(W_{i}, W_{j}\right)-d\left(V_{i}, V_{j}\right)\right|>\eta$ is at most $\eta k^{2}$. Since $V_{1}, \ldots, V_{k}$ is an equipartition into nonempty parts, at most $\eta k^{2} \cdot 4\left(\frac{n}{k}\right)^{2}=4 \eta n^{2}$ 
edges are changed between such pairs. In total at most $2 \eta\left(\begin{array}{l}n \\ 2\end{array}\right)+4 \eta n^{2} \leq 5 \eta n^{2}<\epsilon n^{2}$ edges were changed in order to obtain $G^{\prime}$ from $G$.

From Lemma 5.11, we have $\delta^{\prime}=\frac{1}{8 S^{2}}$, where $S=S(\alpha, s, f)$ is the function from Lemma 5.10 with $\alpha=\frac{\eta^{6}}{4}, s=2 \eta^{-1}$ and $f(k)=\frac{1}{4 h} \eta^{h}$. From Lemma 5.10, $S(\alpha, s, f)$ will be at most a tower in $h$ whose height is proportional to $\eta^{-6}$. Therefore, by the choice of $\eta$ and $\delta$ in the above proof of the induced graph removal lemma, we indeed get the desired tower-type bound. This also completes the proof of Theorem 1.3 .

\section{Regular approximation lemma}

In this section we show how to derive the regular approximation lemma from Tao's regularity lemma, as discussed in Subsection 1.5. The key lemma is Lemma 6.1, which shows how to turn a bipartite graph into a regular pair by changing some edges according to a weak regular partition. We use the notation $x=y \pm \epsilon$ to denote the fact that $y-\epsilon \leq x \leq y+\epsilon$.

It will be helpful to use the Hoeffding-Azuma inequality for concentration of measure. Say that a random variable $X(\omega)$ on an $n$-dimensional product space $\Omega=\prod_{i=1}^{n} \Omega_{i}$ is Lipschitz if changing $\omega$ in any single coordinate affects the value of $X(\omega)$ by at most one. The Hoeffding-Azuma inequality (see, e.g., [10]) provides concentration for these distributions.

Theorem 6.1 (Hoeffding-Azuma Inequality) Let $X$ be a Lipschitz random variable on an $n$ dimensional product space. Then for any $t \geq 0$,

$$
\mathbb{P}[|X-\mathbb{E}[X]|>t] \leq 2 \exp \left\{-\frac{t^{2}}{2 n}\right\} .
$$

For a bipartite graph across parts $A$ and $B$, and partitions $\mathcal{A}: A=A_{1} \cup \ldots \cup A_{r}$ and $\mathcal{B}: B=$ $B_{1} \cup \ldots \cup B_{s}$, let $q(A, B)=d^{2}(A, B)$ and $q(\mathcal{A}, \mathcal{B})=\sum_{i, j} \frac{\left|A_{i}\right|\left|B_{j}\right|}{|A||B|} d^{2}\left(A_{i}, B_{j}\right)$ be the mean square density across the partitions $\mathcal{A}$ and $\mathcal{B}$.

Lemma 6.1 Let $0<\delta<1$. Suppose $A, B$ are disjoint vertex subsets of a graph with $|A| \geq|B|>8 \delta^{-2}$ and $d(A, B)=\eta$. Suppose further that $\mathcal{A}: A=A_{1} \cup \ldots \cup A_{r}$ and $\mathcal{B}: B=B_{1} \cup \ldots \cup B_{s}$ form a weak $\delta$-regular partition of the pair $(A, B)$, i.e., for all $S \subset A$ and $T \subset B$, we have

$$
\left|\sum_{i=1}^{r} \sum_{j=1}^{s}\right| A_{i} \cap S|| B_{j} \cap T\left|d\left(A_{i}, B_{j}\right)-d(S, T)\right| S|| T|| \leq \delta|A||B| .
$$

Then, one can add or remove at most $\left(\delta+(q(\mathcal{A}, \mathcal{B})-q(A, B))^{1 / 2}\right)|A||B|$ edges across $(A, B)$ and thus turn it into a $2 \delta^{1 / 3}$-regular pair satisfying $d(A, B)=\eta \pm \delta$.

Proof: Let $\alpha_{i, j}=d\left(A_{i}, B_{j}\right)-d(A, B)$. If $\alpha_{i, j} \geq 0$, we delete each of the edges connecting $A_{i}$ and $B_{j}$ independently with probability $\frac{\alpha_{i, j}}{d\left(A_{i}, B_{j}\right)}$. If $\alpha_{i, j}<0$, we add each of the nonedges between $A_{i}$ and $B_{j}$ 
with probability $-\frac{\alpha_{i, j}}{1-d\left(A_{i}, B_{j}\right)}$. Clearly the expected value of $d(A, B)$ after these modifications is $\eta$. By the Hoeffding-Azuma inequality, the probability that the new density deviates from $\eta$ by more than $\delta$ is at most

$$
2 \exp \left\{-\frac{(\delta|A||B|)^{2}}{2|A||| B \mid}\right\}=2 \exp \left\{-\delta^{2}|A||B| / 2\right\}<1 / 4 .
$$

Also, the expected number of edges changed is

$$
\begin{aligned}
\sum_{i, j}\left|\alpha_{i, j}\right|\left|A_{i}\right|\left|B_{j}\right| & =\sum_{i, j}\left|d\left(A_{i}, B_{j}\right)-d(A, B)\right|\left|A_{i}\right|\left|B_{j}\right|=|A||B| \sum_{i, j}\left|d\left(A_{i}, B_{j}\right)-d(A, B)\right| p_{i} q_{j} \\
& \leq|A||B|\left(\sum_{i, j}\left(d\left(A_{i}, B_{j}\right)-d(A, B)\right)^{2} p_{i} q_{j}\right)^{1 / 2}=|A||B|(q(\mathcal{A}, \mathcal{B})-q(A, B))^{1 / 2}
\end{aligned}
$$

where $p_{i}=\left|A_{i}\right| /|A|$ and $q_{j}=\left|B_{j}\right| /|B|$ and in the inequality we used the Cauchy-Schwarz inequality. By the Hoeffding-Azuma inequality, the probability that the number of edges changed deviates by more than $\delta|A||B|$ from its expected value is at most

$$
2 \exp \left\{-\frac{(\delta|A||B|)^{2}}{2|A||B|}\right\}=2 \exp \left\{-\delta^{2}|A||B| / 2\right\}<1 / 4 .
$$

Consider now two subsets $A^{\prime} \subset A$ and $B^{\prime} \subset B$. As $(A, B)$ was initially weak $\delta$-regular, the expected value of $e\left(A^{\prime}, B^{\prime}\right)$ differs from $\eta\left|A^{\prime}\right|\left|B^{\prime}\right|$ by at most $\delta|A||B|$. By the Hoeffding-Azuma inequality, we get that the probability that $e\left(A^{\prime}, B^{\prime}\right)$ deviates from its expected value by more than $\delta|A||B|$ is at most

$$
2 \exp \left\{-\frac{(\delta|A||B|)^{2}}{2\left|A^{\prime}\right|\left|B^{\prime}\right|}\right\} \leq 2 \exp \left\{-\delta^{2}|A||B| / 2\right\}<2 \exp \{-4|A|\} \leq 2^{-|A|-|B|-2}
$$

where we use $|A| \geq|B|>8 \delta^{-2}$. As there are $2^{|A|+|B|}$ choices for $\left(A^{\prime}, B^{\prime}\right)$, we get that with probability at least $3 / 4$, all pairs $\left(A^{\prime}, B^{\prime}\right)$ are within $2 \delta|A||B|$ edges of having edge density $\eta$. To recap, we get that with probability at least $1 / 4$ we made at most $\left(\delta+(q(\mathcal{A}, \mathcal{B})-q(A, B))^{1 / 2}\right)|A||B|$ edge modifications, $d(A, B)=\eta \pm \delta$ and all subsets $A^{\prime} \subset A, B^{\prime} \subset B$ are within $2 \delta|A||B|$ edges from having edge density $\eta$. Hence, there is such a choice for these edge modifications, and we claim that this implies that the pair $(A, B)$ is $2 \delta^{1 / 3}$-regular. Indeed, otherwise there would be $A^{\prime} \subset A, B^{\prime} \subset B$, with $\left|A^{\prime}\right| \geq$ $2 \delta^{1 / 3}|A|,\left|B^{\prime}\right| \geq 2 \delta^{1 / 3}|B|$, and $\left|d\left(A^{\prime}, B^{\prime}\right)-d(A, B)\right|>2 \delta^{1 / 3}$, which implies that $A^{\prime}, B^{\prime}$ differs by at least $2 \delta^{1 / 3}\left|A^{\prime}\right|\left|B^{\prime}\right| \geq\left(2 \delta^{1 / 3}\right)^{3}|A||B|=8 \delta|A||B|$ edges from having edge density $d(A, B)$, a contradiction. This completes the proof.

We next use Lemma 6.1 to deduce the regular approximation lemma from Tao's regularity lemma.

Proof: Let $\delta: \mathbb{N} \rightarrow(0,1)$ be defined by $\delta(t)=\min \left(\frac{g(t)^{3}}{32 t^{2}}, \epsilon / 2\right)$. Let $\epsilon_{0}=(\epsilon / 2)^{2}$. Let $T_{0}=T_{0}\left(\delta, \epsilon_{0}, s\right)$ be the bound on the number of parts in Tao's regularity lemma and $T=16 T_{0} / \delta\left(T_{0}\right)^{2}$. If the number $n$ of vertices of the graph $G$ satisfies $n \leq T$, then we can partition $G$ into parts of size one, and the desired conclusion is satisfied in this case. Hence, we may assume $n>T$. By Tao's regularity lemma, the graph $G$ has an equitable vertex partition $P$ into $t \geq s$ parts and an equitable vertex refinement $Q$ into at most $T_{0}$ parts which is weak $\delta(t)$-regular such that $q(Q) \leq q(P)+\epsilon_{0}$. 
For each pair of parts $(A, B)$ of partition $P$, let $\mathcal{A}$ and $\mathcal{B}$ denote the partitions of $A$ and $B$ given by partition $Q$. Since $Q$ is a weak $\delta(t)$-regular partition, and $A$ and $B$ have cardinality at least $\left\lfloor\frac{n}{t}\right\rfloor \geq \frac{n}{2 t}$, then the partitions $\mathcal{A}$ and $\mathcal{B}$ form a weak $4 t^{2} \delta(t)$-regular partition. Note that $4 t^{2} \delta(t) \leq \frac{g(t)^{3}}{8}$. Since $|A|,|B| \geq \frac{n}{2 t}>8 / \delta(t)^{2}$, we may apply Lemma 6.1 to the graph between $A$ and $B$. That is, we may change at most $\left(\delta(t)+(q(\mathcal{A}, \mathcal{B})-q(A, B))^{1 / 2}\right)|A||B|$ edges across $A$ and $B$ and, in so doing,

make $(A, B)$ a $g(t)$-regular pair, where we used that $g(t)=2\left(\frac{g(t)^{3}}{8}\right)^{1 / 3}$. In total, the number of edges we change to obtain a graph $G^{\prime}$ which is $g$-regular with respect to partition $P$ is at most

$$
\sum_{A, B \in P}\left(\delta(t)+(q(\mathcal{A}, \mathcal{B})-q(A, B))^{1 / 2}\right)|A||B| \leq\left(\delta(t)+\epsilon_{0}^{1 / 2}\right) n^{2} \leq \epsilon n^{2}
$$

where we used Jensen's inequality for the concave function $h(x)=x^{1 / 2}$, the inequality $q(Q) \leq q(P)+\epsilon_{0}$, and the bounds $\delta(t) \leq \epsilon / 2, \epsilon_{0}=(\epsilon / 2)^{2}$. To complete the proof, we recall that the number of parts in partition $P$ is at least $s$ and at most $T_{0}=T_{0}\left(\delta, \epsilon_{0}, s\right)$.

\section{Frieze-Kannan weak regularity lemma}

In this section we prove Theorem 1.4 which provides a lower bound on the weak regularity lemma. For a vertex partition $P: V=V_{1} \cup \ldots \cup V_{k}$ of a graph $G=(V, E)$, let

$$
f_{P}(A, B)=f_{P}^{G}(A, B)=e(A, B)-\sum_{1 \leq i, j \leq k} d\left(V_{i}, V_{j}\right)\left|A \cap V_{i}\right|\left|B \cap V_{j}\right|
$$

which is the difference between the number of edges between $A$ and $B$ and the expected number of edges based on the densities across the pairs of parts of the partition and the intersection sizes of $A$ and $B$ with the parts. We call a partition $P$ of the vertex set of a graph $G=(V, E)$ weak $\epsilon$-regular if it satisfies

$$
\left|f_{P}(A, B)\right| \leq \epsilon|V|^{2}
$$

for all $A, B \in V$. Recall that the weak regularity lemma states that for each $\epsilon>0$ there is a positive integer $k(\epsilon)$ such that every graph has an equitable weak $\epsilon$-partition into at most $k(\epsilon)$ parts. Moreover, one may take $k(\epsilon)=2^{O\left(\epsilon^{-2}\right)}$. We will prove that the number of parts required in the weak regularity lemma satisfies $k(\epsilon)=2^{\Omega\left(\epsilon^{-2}\right)}$, thus matching the upper bound.

The following simple lemma of Frieze and Kannan (see Lemma 7(a) of [20]) shows that the notion of weak regularity is robust.

Lemma 7.1 If a partition is weak $\epsilon$-regular, then any refinement of it is weak $2 \epsilon$-regular.

The robustness of weak regularity described by Lemma 7.1 is not shared by the usual notion of regular partition. For example, for any fixed $\epsilon>0$ and positive integer $t$, almost surely any partition into $t$ parts of a uniform random graph on sufficiently many vertices is $\epsilon$-regular, while any partition of the 
vertex set into parts of size 2 is not $(\epsilon, \delta, \eta)$-regular with $\epsilon=1, \delta=\eta=1 / 2$. This is because almost surely in any such partition, between most pairs of parts of size 2 , there will be at least one edge and at least one nonedge.

What we will actually prove is the stronger result that any weak $\epsilon$-regular partition must have $2^{\Omega\left(\epsilon^{-2}\right)}$ parts, whether it is equitable or not. The corresponding regularity lemma, which is an immediate corollary of the usual weak regularity lemma, is the following.

Lemma 7.2 For each $\epsilon>0$ there is a positive integer $k^{*}(\epsilon)$ such that every graph $G=(V, E)$ has a vertex partition $P$ with at most $k^{*}(\epsilon)$ parts which is weak $\epsilon$-regular.

In the other direction, the equitable version of the weak regularity lemma also follows from Lemma 7.2 . This is because of the robustness property discussed in Lemma 7.1 above, that is, any refinement of a weak $\epsilon$-regular partition is a $2 \epsilon$-regular partition. By arbitrarily refining a not necessarily equitable partition into an equitable partition (except for a small fraction of vertices, which we distribute evenly amongst the other parts), we get an equitable weak $3 \epsilon$-partition whose number of parts is only a factor polynomial in $\epsilon^{-1}$ larger.

In order to prove the lower bound for weak regularity, we will need to perform some further reductions. We first state a bipartite variant which can easily be shown to be equivalent to Lemma 7.2, For a bipartite graph $G=(U, V, E)$ with $|U|=|V|=n$, partitions $P_{1}: U=U_{1} \cup \ldots \cup U_{k}$ and $P_{2}: V=$ $V_{1} \cup \ldots \cup V_{k^{\prime}}$, and vertex subsets $A \subset U$ and $B \subset V$, let

$$
f_{P_{1}, P_{2}}(A, B)=f_{P_{1}, P_{2}}^{G}(A, B)=e(A, B)-\sum_{i=1}^{k} \sum_{j=1}^{k^{\prime}} d\left(U_{i}, V_{j}\right)\left|A \cap U_{i}\right|\left|B \cap V_{j}\right| .
$$

We call the pair of partitions $P_{1}, P_{2}$ weak $\epsilon$-regular with respect to the bipartite graph $G$ if

$$
\left|f_{P_{1}, P_{2}}(A, B)\right| \leq \epsilon n^{2}
$$

for all $A \subset U$ and $B \subset V$.

Lemma 7.3 For each $\epsilon>0$ there is a positive integer $k^{\prime}(\epsilon)$ such that every bipartite graph $G=$ $(U, V, E)$ with parts of equal size has partitions $P_{1}$ of $U$ and $P_{2}$ of $V$ each with at most $k^{\prime}(\epsilon)$ parts which form a weak $\epsilon$-regular partition.

To prove Theorem 1.4, it suffices to show $k^{\prime}(\epsilon)=2^{\Omega\left(\epsilon^{-2}\right)}$. Indeed, this follows from the bound $k^{\prime}(\epsilon) \leq k^{*}(\epsilon / 2)$. This inequality follows from first considering a single weak $\epsilon / 2$-regular partition $P$ for the bipartite graph $G$ into at most $k^{*}(\epsilon / 2)$ parts, and then refining it into a partition $P^{\prime}$ with at most $2 k^{*}(\epsilon / 2)$ parts based on the intersections of the parts of $P$ with $U$ and $V$. By Lemma 7.1, $P^{\prime}$ is a weak $\epsilon$-regular partition. Letting $P_{1}$ be the parts of $P^{\prime}$ in $U$ and $P_{2}$ be the parts of $P^{\prime}$ in $V$, the pair $P_{1}, P_{2}$ form a weak $\epsilon$-regular partition, each with at most $k^{*}(\epsilon / 2)$ parts, so that $k^{\prime}(\epsilon) \leq k^{*}(\epsilon / 2)$.

To get a lower bound for the weak regularity lemma, we do not need to show the other direction of the equivalence between the weak regularity lemma and Lemma 7.3, that Lemma 7.3 implies the 
weak regularity lemma. However, this is rather simple, so we sketch it here. From a graph $G$ we can consider the bipartite double cover of $G$, which is the tensor product of $G$ with $K_{2}$. Applying Lemma 7.3, we get a pair $P_{1}, P_{2}$ of partitions of $V(G)$ which form a weak $\epsilon / 2$-regular partition with respect to the bipartite double cover of $G$. Refining the two partitions $P_{1}, P_{2}$ of $V(G)$, we get by Lemma 7.1 a weak $\epsilon$-regular partition for $G$, thus establishing $k^{*}(\epsilon) \leq k^{\prime}(\epsilon / 2)^{2}$.

The following technical lemma will allow us to construct a weighted graph rather than a graph. A similar idea is present in the lower bound construction of Gowers [22] for Szemerédi's regularity lemma. Let $W$ be a $[0,1]$-valued $n \times n$ matrix. We view $W$ as a weighted graph with parts $U$ and $V$, where $U$ and $V$ denote the set of columns and rows, respectively, of $W$. Let $e_{W}(A, B)=\sum_{a \in A, b \in B} W(a, b)$ and $d_{W}(A, B)=\frac{e_{W}(A, B)}{|A||B|}$.

Lemma 7.4 Let $M$ be an $n \times n$ matrix with entries in the interval $[0,1]$. Let $G=(U, V, E)$ be a bipartite random graph with $|U|=|V|=n$ and edges chosen independently given by $M$ and let $\theta=4 n^{-1 / 2}$. With probability at least $1-e^{-4 n}$, we have $\left|e_{M}(A, B)-e_{G}(A, B)\right| \leq \theta n^{2}$ for every pair of sets $A \subset U, B \subset V$.

Proof: Given two vertices $u \in U$ and $v \in V$, let $a(u, v)$ be the random variable $G(u, v)-M(u, v)$ (where $G$ has been identified with its adjacency matrix). The mean of $a(u, v)$ is zero for all $u, v$ and the modulus of $a(u, v)$ is at most 1 . Hence, by the Hoeffding-Azuma inequality (Theorem 6.1), given two sets $A \subset U$ and $B \subset V$, the probability that

$$
\left|\sum_{(u, v) \in A \times B} a(u, v)\right| \geq \theta n^{2}
$$

is at most $2 \exp \left\{-\left(\theta n^{2}\right)^{2} /(2|A||B|)\right\} \leq 2 \exp \{-8 n\}$. Summing over all $A \subset U$ and $B \subset V$, the probability that there are subsets $A \subset U$ and $B \subset V$ with $\left|e_{G}(A, B)-e_{M}(A, B)\right| \geq \theta n^{2}$ is at most $2^{2 n} \cdot 2 e^{-8 n} \leq e^{-4 n}$.

For partitions $P_{1}: U=U_{1} \cup \ldots \cup U_{k}$ and $P_{2}: V=V_{1} \cup \ldots \cup V_{k^{\prime}}$, let

$$
f_{P_{1}, P_{2}}(A, B)=e_{W}(A, B)-\sum_{i=1}^{k} \sum_{j=1}^{k^{\prime}} d_{W}\left(U_{i}, V_{j}\right)\left|U_{i} \cap A\right|\left|V_{j} \cap B\right| .
$$

We say that partitions $P_{1}, P_{2}$ form a weak $\epsilon$-regular partition of $W$ if $\left|f_{P_{1}, P_{2}}(A, B)\right| \leq \epsilon n^{2}$ for all subsets $A \subset U$ and $B \subset V$.

Corollary 7.1 Suppose $W=(U, V, E)$ is an edge-weighted graph with weights in $[0,1]$ and $|U|=$ $|V|=n$. Let $G=(U, V, E)$ be a bipartite random graph with $|U|=|V|=n$ and edges chosen independently given by $W$ and let $\theta=4 n^{-1 / 2}$. With probability at least $1-e^{-4 n}$, every pair of partitions $P_{1}: U=U_{1} \cup \ldots \cup U_{k}$ and $P_{2}: V=V_{1} \cup \ldots \cup V_{k^{\prime}}$ which form a weak $\epsilon$-partition for $G$ also form a weak $(\epsilon+2 \theta)$-regular partition for $W$. 
Proof: By Lemma 7.4, with probability at least $1-e^{-4 n}$, we have $\left|e_{G}(A, B)-e_{W}(A, B)\right| \leq \theta n^{2}$ for every pair of vertex subsets $A \subset U$ and $B \subset V$. Suppose this indeed holds. For graph $G$, we have

$$
f_{P_{1}, P_{2}}^{G}(A, B)=e_{G}(A, B)-\sum_{i=1}^{k} \sum_{j=1}^{k^{\prime}} d_{G}\left(U_{i}, V_{j}\right)\left|A \cap U_{i}\right|\left|B \cap V_{j}\right| .
$$

The first term is within $\theta n^{2}$ of $e_{W}(A, B)$. The second term is the average of $e_{G}\left(A^{\prime}, B^{\prime}\right)$ over all subsets $A^{\prime} \subset U$ and $B^{\prime} \subset V$ with $\left|A^{\prime} \cap U_{i}\right|=\left|A \cap U_{i}\right|$ for all $i$ and $\left|B^{\prime} \cap V_{j}\right|=\left|B \cap V_{j}\right|$ for all $j$, and hence is within $\theta n^{2}$ of the corresponding average of $e_{W}\left(A^{\prime}, B^{\prime}\right)$ over all of the same pairs $\left(A^{\prime}, B^{\prime}\right)$. By the triangle inequality, for $W$, we get that for all $A \subset U$ and $B \subset V$, we have $\left|f_{P_{1}, P_{2}}^{W}(A, B)\right| \leq$ $\left|f_{P_{1}, P_{2}}^{G}(A, B)\right|+2 \theta n^{2} \leq(\epsilon+2 \theta) n^{2}$. Hence, $P_{1}, P_{2}$ also form a weak $(\epsilon+2 \theta)$-regular partition for $W$.

From Corollary 7.1 and the previous remarks, to obtain the desired lower bound in Theorem 1.4 on the number of parts in the weak regularity lemma it suffices to prove a lower bound of the form $2^{\Omega\left(\epsilon^{-2}\right)}$ on the number of parts $k_{0}(\epsilon)$ in the following weak regularity lemma for weighted bipartite graphs.

Lemma 7.5 For each $\epsilon>0$ there is a positive integer $k_{0}(\epsilon)$ such that every edge-weighted bipartite graph $G=(U, V, E)$ with weights in $[0,1]$ and parts of equal size has partitions $P_{1}$ of $U$ and $P_{2}$ of $V$ each with at most $k_{0}(\epsilon)$ parts which form a weak $\epsilon$-regular partition.

Lemma 7.5 is also known as the weak matrix regularity lemma. This is because it provides, for any $n \times n$ matrix with entries in $[0,1]$, partitions of the rows and columns into a bounded number of parts, such that the sum of the entries in any submatrix (which is the product of a set of rows and columns) is within $\epsilon n^{2}$ of what is expected based on the intersections with the parts and the density between the parts.

Our goal is to construct a bipartite graph $G$ with edge weights in $[0,1]$ which provides a lower bound of the form $k_{0}(\epsilon)=2^{\Omega\left(\epsilon^{-2}\right)}$. Suppose $0<\epsilon \leq 2^{-50}$. Consider the following weighted bipartite graph $G$. The graph has parts $U$ and $V$ each of order $n=2^{2^{-45} \epsilon^{-2}}$. Let $r=2^{-40} \epsilon^{-2}$ and $\alpha=2^{14} \epsilon$. Consider, for $1 \leq i \leq r$, equitable partitions $U=U_{0}^{i} \cup U_{1}^{i}$ and $V=V_{0}^{i} \cup V_{1}^{i}$ picked uniformly and independently at random. For vertices $u \in U$ and $v \in V$, let $s(u, v)$ be the number of $i \in[r]$ for which there is $j \in\{0,1\}$ such that $u \in U_{j}^{i}$ and $v \in V_{j}^{i}$, and $t(u, v)$ be the number of $i \in[r]$ for which there is $j \in\{0,1\}$ such that $u \in U_{j}^{i}$ and $v \in V_{1-j}^{i}$, so that $s(u, v)+t(u, v)=r$. Let $W(u, v)=\frac{1}{2}+(s(u, v)-t(u, v)) \alpha$. We define the weight $w(u, v)$ between $u$ and $v$ as follows. If $0 \leq W(u, v) \leq 1$, then $w(u, v)=W(u, v)$, if $W(u, v)<0$, then $w(u, v)=0$, and if $W(u, v)>1$, then $w(u, v)=1$.

Call a pair $(u, v) \in U \times V$ extreme if $|W(u, v)-1 / 2|>1 / 4$, and a vertex $u \in U$ nice if it is in at most $n / 8$ pairs $(u, v)$ with $v \in V$ which are extreme.

Lemma 7.6 With probability at least $3 / 4$, all but at most $e^{-100} n$ vertices of $U$ are nice.

Proof: Fix a pair $(u, v) \in U \times V$. The event $(u, v)$ is extreme is the same as $|s(u, v)-t(u, v)| \alpha>1 / 4$, or equivalently that $|s(u, v)-r / 2|>\frac{1}{8 \alpha}$. The number $s(u, v)$ is a sum of $r$ independent variables, with 
values 0 or 1 each with probability $1 / 2$, and hence follows a binomial distribution with mean $r / 2$. By Chernoff's bound (11), the probability that $|s(u, v)-r / 2|>\frac{1}{8 \alpha}$ is less than $2 e^{-2(1 /(8 \alpha))^{2} / r}=2 e^{-2^{7}}$. Hence, by linearity of expectation, the expected number of extreme pairs $(u, v) \in U \times V$ is less than $2 e^{-2^{7}} n^{2}$. Therefore, by Markov's inequality, the probability that there are at least $8 e^{-2^{7}} n^{2}$ extreme pairs $(u, v)$ is less than $1 / 4$. Since any nice vertex is contained in at most $n / 8$ extreme pairs, we see that with probability at least $3 / 4$, all but at most $64 e^{-2^{7}} n \leq e^{-100} n$ vertices in $U$ are nice.

For $h \in[r]$, we let $s_{h}(u, v)$ denote the number of $i \in[r] \backslash\{h\}$ for which there is $j \in\{0,1\}$ such that $u \in U_{j}^{i}$ and $v \in V_{j}^{i}$, and $t_{h}(u, v)$ be the number of $i \in[r] \backslash\{h\}$ for which there is $j \in\{0,1\}$ such that $u \in U_{j}^{i}$ and $v \in V_{1-j}^{i}$, so that $s_{h}(u, v)+t_{h}(u, v)=r-1$. Let $W_{h}(u, v)=\frac{1}{2}+\left(s_{h}(u, v)-t_{h}(u, v)\right) \alpha$. As above, we define the weight $w_{h}(u, v)$ by $w_{h}(u, v)=W_{h}(u, v)$ if $0 \leq W_{h}(u, v) \leq 1, w_{h}(u, v)=0$ if $W_{h}(u, v)<0$, and $w_{h}(u, v)=1$ if $W_{h}(u, v)>1$.

Lemma 7.7 Suppose $u \in U_{j}^{h}$ and we are given $\left|w_{h}(u, v)-1 / 2\right| \leq 1 / 2-\alpha$ for at least $\frac{7}{8} n$ vertices $v \in V$, and we do not yet know the partition $V=V_{0}^{h} \cup V_{1}^{h}$. Then the probability that $d_{w}\left(u, V_{j}^{h}\right)-d_{w}\left(u, V_{1-j}^{h}\right) \geq$ $\alpha / 2$ is at least $1-\frac{1}{4 r n}$.

Proof: Consider the event $E$ that

$$
\sum_{v \in V_{1-j}^{h}} w_{h}(u, v)-\sum_{v \in V_{j}^{h}} w_{h}(u, v) \geq \alpha n / 4 .
$$

Note that the expected value of the left hand side is 0 . Recall that a hypergeometric distribution is at least as concentrated as the sum of independent random variables with the same values (for a proof, see Section 6 of [25]). By the Hoeffding-Azuma inequality (Theorem 6.1), the probability of event $E$ is at most

$$
2 \exp \left\{-\frac{(\alpha n / 8)^{2}}{2 n}\right\}=2 \exp \left\{-2^{-7} \alpha^{2} n\right\} \leq \frac{1}{4 r n},
$$

where in the last inequality we use $0<\epsilon \leq 2^{-50}, r=2^{-40} \epsilon^{-2}, n=2^{2^{-45} \epsilon^{-2}}$, and $\alpha=2^{14} \epsilon$.

For a fixed $u$, if $\left|w_{h}(u, v)-\frac{1}{2}\right| \leq \frac{1}{2}-\alpha$, then $w(u, v)=w_{h}(u, v)+\alpha$ if $v$ is in $V_{j}^{h}$ and $w(u, v)=w_{h}(u, v)-\alpha$ if $v$ is in $V_{1-j}^{h}$. For all $v, w(u, v)$ is within $\alpha$ of $w_{h}(u, v)$. Therefore, letting $w(u, S)=\sum_{s \in S} w(u, s)$, we see, since $\left|w_{h}(u, v)-\frac{1}{2}\right| \leq \frac{1}{2}-\alpha$ for at least $\frac{7}{8} n$ vertices of $v$, that

$$
w\left(u, V_{j}^{h}\right)-w\left(u, V_{1-j}^{h}\right) \geq \frac{7}{8} \alpha n-\frac{1}{8} \alpha n+w_{h}\left(u, V_{j}^{h}\right)-w_{h}\left(u, V_{1-j}^{h}\right) \geq \frac{3}{4} \alpha n-\frac{1}{4} \alpha n=\frac{\alpha}{2} n .
$$

The result follows.

Call a nice vertex $u \in U$ very nice if for each $h \in[r]$ and $j \in\{0,1\}$ with $u \in U_{j}^{h}$,

$$
d\left(u, V_{j}^{h}\right)-d\left(u, V_{1-j}^{h}\right) \geq \alpha / 2 .
$$

Corollary 7.2 With probability at least $3 / 4$, every nice vertex $u$ is very nice. 
Proof: Given $u$ is nice, then for each $h \in[r]$, we must have $\left|W_{h}(u, v)-1 / 2\right| \leq 1 / 2-\alpha$ for all but at most $\frac{7}{8} n$ vertices $v \in V$. The probability that there is a vertex which is nice but not very nice is by Lemma 7.7 at most $r n \cdot \frac{1}{4 r n} \leq 1 / 4$, which completes the proof.

From Lemma 7.6 and Corollary [7.2, we have the following corollary.

Corollary 7.3 With probability at least 1/2, the graph $G$ has the following properties.

- The number of vertices in $U$ which are not nice is at most $e^{-100} n$.

- Every nice vertex is very nice.

Consider the random bipartite graph $B=B(n, r)$ with vertex parts $U$ and $[r]$ where $i \in[r]$ is adjacent to $u \in U$ if $u \in U_{0}^{i}$. By Lemma 2.1 with $\mu=1 / 4$, as $r \geq 32 \log n$, we have the following proposition.

Corollary 7.4 With probability at least $3 / 4$, for each pair $u, u^{\prime} \in U$, the number of $i$ for which $u$ and $u^{\prime}$ both belong to $U_{j}^{i}$ for some $j \in\{0,1\}$ is less than $\frac{3}{4} r$.

Hence, with probability at least 1/4, the graph $G$ satisfies the properties in Corollaries 7.3 and 7.4 . Fix such a graph $G$.

Theorem 7.1 No partitions $P_{1}: U_{1} \cup \ldots \cup U_{k}$ of $U$ and $P_{2}: V_{1} \cup \ldots \cup V_{k^{\prime}}$ of $V$ with $k \leq n / 2$ form a weak $\epsilon$-regular partition. As $n / 2 \geq 2^{2^{-46} \epsilon^{-2}}$, we therefore have $k_{0}(\epsilon)>2^{2^{-46} \epsilon^{-2}}$ for $0<\epsilon \leq 2^{-50}$.

Theorem 7.1 gives a lower bound on the number $k_{0}(\epsilon)$ of parts for the weak matrix regularity lemma (Lemma 7.5) with approximation $\epsilon$. Before we prove this theorem, we remark that it has no restriction on the number of parts of partition $P_{2}$, and further shows that $P_{1}$ has to be almost the finest partition (partition into singletons) to obtain a pair of partitions which are weak $\epsilon$-regular.

Proof: Suppose for contradiction that the partitions $P_{1}$ and $P_{2}$ are weak $\epsilon$-regular. That is, $\left|f_{P_{1}, P_{2}}(A, B)\right| \leq \epsilon n^{2}$ for all subsets $A \subset U$ and $B \subset V$.

Fix for now $U_{t}$ with $\left|U_{t}\right| \geq 2$. Call the pair $(i, t) \in[r] \times[k]$ useful if $\left|U_{t} \cap U_{j}^{i}\right| \geq\left|U_{t}\right| / 32$ for $j \in\{0,1\}$. Let $M_{t}$ be the number of $i \in[r]$ for which the pair $(i, t)$ is useful. The sum

$$
S_{t}=\sum_{i \in[r]}\left|U_{t} \cap U_{0}^{i}\right|\left|U_{t} \cap U_{1}^{i}\right| \leq r\left|U_{t}\right|^{2} / 32+M_{t}\left|U_{t}\right|^{2} / 4
$$

is precisely the number of triples $u, u^{\prime}, i$ with $u, u^{\prime}$ distinct elements of $U_{t}$ and $i \in[r]$ for which $u$ and $u^{\prime}$ are not in the same set in the partition $U=U_{0}^{i} \cup U_{1}^{i}$. By Corollary 17.4, the sum $S_{t}$ is at least $\frac{1}{4} r\left(\begin{array}{c}\left|U_{t}\right| \\ 2\end{array}\right) \geq\left|U_{t}\right|^{2} r / 16$. Hence,

$$
r\left|U_{t}\right|^{2} / 32+M_{t}\left|U_{t}\right|^{2} / 4 \geq S_{t} \geq\left|U_{t}\right|^{2} r / 16 .
$$

We thus have $M_{t} \geq r / 8$. 
Since $M_{t} \geq r / 8$ for each $t$ for which $\left|U_{t}\right| \geq 2$ and there are at most $k$ parts in partition $P_{1}$ of order 1 , there is an $i$ for which partition $i$ satisfies that at least $(n-k) / 8 \geq n / 16$ vertices $u \in U$ are in $U_{t}$ with the pair $(i, t)$ useful. Fix such an $i$. For each $t$ for which $(i, t)$ is useful and all but at most $\left|U_{t}\right| / 64$ vertices in $U_{t}$ are nice, for $j \in\{0,1\}$, let $U_{t, j}$ be a subset of $U_{t} \cap U_{j}^{i}$ of cardinality exactly $\left\lceil\left|U_{t}\right| / 64\right\rceil$, and $A_{j}$ denote the union of all such $U_{t, j}$. Recall from Corollary 7.3 that there are at most $e^{-100} n$ vertices in $U$ which are not nice. Hence, there are at most $64 \cdot e^{-100} n$ vertices in $U$ which belong to a $U_{t}$ for which the pair $(i, t)$ is useful but there are at least $\left|U_{t}\right| / 64$ vertices in $U_{t}$ which are not nice. Thus, the number of vertices in $U$ which belong to a $U_{t}$ for which $(i, t)$ is useful and there are at most $\left|U_{t}\right| / 64$ vertices in $U_{t}$ which are not nice is at least

$$
\frac{n}{16}-64 e^{-100} n>\frac{n}{32} \text {. }
$$

We thus have $\left|A_{0}\right|=\left|A_{1}\right|>\frac{n}{32} / 64=2^{-11} n$.

Note that, by construction, we have for each $t \in[k], \ell \in\left[k^{\prime}\right]$ and $T \subset V$,

$$
\left|A_{0} \cap U_{t}\right|\left|T \cap V_{\ell}\right| d\left(U_{t}, V_{\ell}\right)=\left|A_{1} \cap U_{t}\right|\left|T \cap V_{\ell}\right| d\left(U_{t}, V_{\ell}\right) .
$$

Thus, if the partitions $P_{1}, P_{2}$ form a weak $\epsilon$-regular partition, we would have to have

$$
\left|e\left(A_{0}, V_{j}^{i}\right)-e\left(A_{1}, V_{j}^{i}\right)\right| \leq 2 \epsilon n^{2} .
$$

for $j \in\{0,1\}$. However, as each $u \in A_{0}$ is in $U_{0}^{i}$ and is very nice, we have

$$
d\left(A_{0}, V_{0}^{i}\right)-d\left(A_{0}, V_{1}^{i}\right) \geq \alpha / 2 .
$$

Since $\left|A_{0}\right|>2^{-11} n$ and $\left|V_{j}^{i}\right|=n / 2$ for $j \in\{0,1\}$, we have

$$
e\left(A_{0}, V_{0}^{i}\right)-e\left(A_{0}, V_{1}^{i}\right)>2^{-13} \alpha n^{2} .
$$

Similarly,

$$
e\left(A_{1}, V_{1}^{i}\right)-e\left(A_{1}, V_{0}^{i}\right)>2^{-13} \alpha n^{2} .
$$

Adding the previous two inequalities, we have

$$
e\left(A_{0}, V_{0}^{i}\right)-e\left(A_{1}, V_{0}^{i}\right)+e\left(A_{1}, V_{1}^{i}\right)-e\left(A_{0}, V_{1}^{i}\right)>2^{-12} \alpha n^{2} .
$$

But, by (29) for $j \in\{0,1\}$, the left hand side of (30) is at most $4 \epsilon n^{2}$ in modulus, contradicting the above inequality and $\alpha=2^{14} \epsilon$. This completes the proof.

Remark: While Theorem 7.1 provides for each $\epsilon$ only one graph which requires at least $2^{\Omega\left(\epsilon^{-2}\right)}$ parts in any weak $\epsilon$-regular pair of partitions, it is a simple exercise to modify the proof to show that all blow-ups of $G$ also satisfy this property, thus obtaining an infinite family of such graphs. For a graph $G$ on $n$ vertices and a positive integer $t$, the blow-up $G(t)$ of $G$ is the graph on $n t$ vertices obtained by replacing each vertex $u$ by an independent set $I_{u}$, and a vertex in $I_{u}$ is adjacent to a vertex in $I_{v}$ if and only if $u$ and $v$ are adjacent. 


\section{Concluding remarks}

\section{- Weak regularity lemmas without irregular pairs}

While proving his famous theorem on arithmetic progressions in dense subsets of the integers, Szemerédi [39] actually developed a regularity lemma which is weaker than what is now commonly known as Szemerédi's regularity lemma [40]. The following version is a strengthening of the original version, as it guarantees that all pairs, instead of all but an $\epsilon$-fraction of pairs, under consideration are $\epsilon$-regular. The key extra ingredient is an application of Lemma 5.6 to redistribute the small fraction of vertices which are not in regular sets.

Lemma 8.1 For each $0<\epsilon<1 / 2$ there are integers $k=k(\epsilon)$ and $K=K(\epsilon)$ such that the following holds. For every graph $G=(V, E)$, there is an equitable partition $V=V_{1} \cup \ldots \cup V_{t}$ into at most $k$ parts such that for each $i, 1 \leq i \leq t$, there is a partition $V=V_{i 1} \cup \ldots \cup V_{i j_{i}}$, with $j_{i} \leq K$, such that for all $1 \leq i \leq t$ and $1 \leq j \leq j_{i}$ the pair $\left(V_{i}, V_{i j}\right)$ is $\epsilon$-regular. Furthermore, $k(\epsilon)=2^{\epsilon^{-C}}$ and $K(\epsilon)=O\left(\epsilon^{-1}\right)$, where $C$ is an absolute constant.

Szemerédi [39] originally gave a triple exponential upper bound on the number of parts in the original regularity lemma, whereas it is now known (see [33]) that the correct bound is single exponential. Through iterative applications, the original regularity lemma was used by Ruzsa and Szemerédi [36] to resolve the (6,3)-problem, and by Szemerédi [38] to establish the upper bound on the Ramsey-Turán problem for $K_{4}$. It is a relatively simple exercise to show that Szemerédi's original regularity lemma implies the Frieze-Kannan weak regularity lemma, but with a bound that is one exponential worse than the tight bound established in the previous section. This can be accomplished by showing that the common refinement of the partitions in the original regularity lemma satisfies the Frieze-Kannan weak regularity lemma.

There are a number of notable properties of Lemma 8.1. First, all pairs $\left(V_{i}, V_{i j}\right)$ under consideration in Lemma 8.1 are regular. In contrast, Theorem 1.1 shows that we must allow for many irregular pairs in Szemerédi's regularity lemma. Second, the bounds are much better than in Szemerédi's regularity lemma. The bounds on the number of parts is only single-exponential, instead of the tower-type bound which appears in the standard regularity lemma. Furthermore, each of the partitions of $V$ have at most $K(\epsilon)=O\left(\epsilon^{-1}\right)$ parts. Indeed, this can be established by first proving any bound on $K(\epsilon)$, and then using the following additive property of regularity to combine parts. Namely, applying Lemma 8.1 with any bound on $K(\epsilon)$ and with $\epsilon^{2} / 4$ in place of $\epsilon$, and, for each $i$, partitioning $V$ into $O\left(\epsilon^{-1}\right)$ parts, each part consisting of the union of parts $V_{i j}$ for which $d\left(V_{i}, V_{i j}\right)$ lies in an interval of length at most $\epsilon / 2$, the following lemma shows that $V_{i}$ together with each part of the new partition forms an $\epsilon$-regular pair.

Lemma 8.2 Let $0<\epsilon<1$ and $\alpha=\epsilon^{2} / 4$. Suppose $A, B_{1}, \ldots, B_{r}$ are disjoint sets satisfying $\left(A, B_{i}\right)$ is $\alpha$-regular for $1 \leq i \leq r$ and $\left|d\left(A, B_{i}\right)-d\left(A, B_{j}\right)\right| \leq \epsilon / 2$ for $1 \leq i, j \leq r$. Then, letting $B=B_{1} \cup \ldots \cup B_{r}$, the pair $(A, B)$ is $\epsilon$-regular. 
To save space, we omit the details of how to prove Lemmas 8.1 and 8.2 .

Another interesting consequence of Lemma 8.1 is that it implies that every graph on $n$ vertices has an $\epsilon$-regular pair in which one part is of size $\Omega(\epsilon n)$ and the other part has size $2^{-\epsilon^{-O(1)}} n$. It is well known (see [27]), that one can find an $\epsilon$-regular pair in which both parts have size $2^{-\epsilon^{-O(1)}} n$. In some applications, having one regular pair is sufficient (see, e.g., [16], 24], [27]), and one obtains much better bounds than by applying Szemerédi's regularity lemma. In the other direction, there are graphs (see Theorem 1.4 in [31] for a tight result) for which any $\epsilon$ regular pair has a part of size $2^{-\epsilon^{-\Omega(1)}} n$. We can nevertheless guarantee that one part is of size $\Omega(\epsilon n)$. It seems likely that having such a large part in a regular pair could be useful in some applications.

By iterative application of Lemma 8.1, one can also obtain a version of the Duke-Lefmann-Rödl lemma such that all cylinders in the partition are $\epsilon$-regular. In fact, using Lemma 5.7, one can further guarantee that all cylinders in the partition are strongly $\epsilon$-regular, and the bound is still of constant-tower height.

\section{- A part irregular with no other parts}

In the proof of Theorem 1.1, we found a graph $G$ such that for any partition into $k$ parts there are at least $\theta k$ parts $V_{i}$ for which there are at least $\theta^{-1} \eta k$ pairs $\left(V_{i}, V_{j}\right)$ which are not $(\epsilon, \delta)$-regular, where $0<\theta<1$ is a fixed constant. Is it possible to improve this result so that all parts are in $\eta k$ irregular pairs? The answer is no, as Lemma 8.1 implies that any graph has an equitable partition into only $2^{\epsilon^{-O(1)}}$ parts in which one part is $\epsilon$-regular with all the other parts.

\section{- A single regular subset}

It would be interesting to determine tight bounds for the size of the largest $\epsilon$-regular subset which can be found in every graph. In Lemma 5.2, we showed that every graph $G=(V, E)$ must contain an $\epsilon$-regular subset $U$ of size at least $2^{-\epsilon^{-(10 / \epsilon)^{4}}}|V|$. On the other hand, a result of Peng, Rödl and Ruciński [31] implies that there are graphs $G=(V, E)$ which contain no $\epsilon$-regular subset of size $\epsilon^{c \epsilon^{-1}}|V|$. We conjecture that the correct bound is single exponential, though the power may be polynomial in $\epsilon^{-1}$.

\section{- Equitable partitions and not necessarily equitable partitions}

In the regularity lemmas considered in this paper, we often assume the partitions we consider are equitable partitions. It is not difficult to see that this assumption is non-essential and the bounds do not change much. Indeed, consider for example the following variant of Szemerédi's regularity lemma.

Lemma 8.3 For each $\epsilon, \delta, \eta>0$ there is a positive integer $M=M(\epsilon, \delta, \eta)$ for which the following holds. Every graph $G=(V, E)$ has a vertex partition $V=V_{1} \cup \ldots \cup V_{k}$ with $k \leq M$ such that the sum of $\left|V_{i}\right|\left|V_{j}\right|$ over all pairs $\left(V_{i}, V_{j}\right)$ which are not $(\epsilon, \delta)$-regular is at most $\eta|V|^{2}$. 
The key difference between this version of Szemerédi's regularity lemma and the usual statement is that the parts of the partition are not necessarily of equal order, and we measure the regularity of the partition by the sum of the products of the sizes of the pairs of parts that are $(\epsilon, \delta)$-regular. Szemerédi's regularity lemma clearly implies Lemma 8.3, as it further specifies that the partition is an equitable partition, and the condition that the sum of $\left|V_{i}\right|\left|V_{j}\right|$ over all pairs $\left(V_{i}, V_{j}\right)$ which are not $(\epsilon, \delta)$-regular is at most $\eta|V|^{2}$ is then essentially the same as saying that the number of pairs $\left(V_{i}, V_{j}\right)$ which are not $(\epsilon, \delta)$-regular is at most $\eta k^{2}$. To see that Lemma 8.3 implies Szemerédi's regularity lemma with similar bounds, first apply Lemma 8.3, and then randomly refine each part $V_{i}=V_{i 0} \cup V_{i 1} \cup \ldots \cup V_{i j_{i}}$ into parts of size $m=\frac{1}{100} \alpha^{2}|V| / M$, where $\alpha=\min (\epsilon, \delta, \eta)$, except possibly $V_{i 0}$, which can have size less than $m$ as not necessarily each $V_{i}$ has cardinality a multiple of $m$. The total number of remaining vertices, those in $V_{0}=\bigcup_{i=1}^{k} V_{i 0}$, is less than $k m \leq \frac{1}{100} \alpha^{2}|V|$, as there are less than $m$ remaining vertices from each of the $k$ parts $V_{i}$. Redistributing the vertices of $V_{0}$ evenly among the parts of size $m$, we get a new equitable partition where each part has size

between $m$ and at most $\left(1+\frac{1}{50} \alpha^{2}\right) m$. It is not hard to show using an additional application of the Frieze-Kannan weak regularity lemma, that because we randomly refined each part, almost surely for all pairs $\left(V_{h}, V_{i}\right)$ which are $(\epsilon, \delta)$-regular, all pairs $\left(V_{h a}, V_{i j}\right)$ are $(2 \epsilon, 2 \delta)$-regular. That is, the regularity between pairs of parts is almost surely inherited between large vertex subsets. It then easily follows that the equitable partition is similar both in the number of parts and the degree of regularity to the original partition from Lemma 8.3 ,

Because of this equivalence, we get similar lower bounds for regularity lemmas where the partitions are not necessarily equitable partitions. For example, for Lemma 8.3, for some absolute constants $\epsilon, \delta>0$, we get a bound on $M(\epsilon, \delta, \eta)$ which is a tower of $2 \mathrm{~s}$ of height $\Omega\left(\eta^{-1}\right)$. Similarly, in Theorem 1.2 and Corollary 1.1 giving lower bounds on the strong regularity lemma, the assumption that $\mathcal{B}$ is an equitable partition is not needed.

Finally, as we have already noted in Section 7, it is much easier to show that for the FriezeKannan weak regularity lemma we do not need to assume that the partition is equitable. This is a simple consequence of the robustness of weak regularity under refinement.

\section{- Irregular pairs and half graphs}

A (generalized) half-graph has vertex set $A \cup B$ with $2 n$ vertices $A=\left\{a_{1}, \ldots, a_{n}\right\}$ and $B=$ $\left\{b_{1}, \ldots, b_{n}\right\}$, in which $\left(a_{i}, b_{j}\right)$ is an edge if and only if $i \leq j$ (but the edges within $A$ and $B$ could be arbitrary). As mentioned in the introduction, any partition of a large half-graph into a constant number of parts has some irregular pairs. Malliaris and Shelah [30] recently showed that for each fixed $k$, every graph on $n$ vertices with no induced subgraph which is a half-graph on $2 k$ vertices has an $\epsilon$-regular partition with no irregular parts and the number of parts is at most $\epsilon^{-c_{k}}$, where $c_{k}$ is single-exponential in $k$. This shows that any construction forcing irregular pairs in the regularity lemma, like that given in the proof of Theorem 1.1. must contain large half-graphs, of size double-logarithmic in the number of vertices.

\section{- Distinct regular approximations}


The notion of $f$-regularity, which appears in the regular approximation lemma, has since appeared elsewhere. Alon, Shapira, and Stav [9] investigate the question of determining if a graph $G=(V, E)$ can have distinct regular partitions. Two equitable partitions $\mathcal{U}: U=U_{1} \cup \ldots \cup U_{k}$ and $\mathcal{V}: V=V_{1} \cup \ldots \cup V_{k}$ into $k$ parts are said to be $\epsilon$-isomorphic if there is a permutation $\pi:[k] \rightarrow[k]$ such that for all but at most $\epsilon\left(\begin{array}{l}k \\ 2\end{array}\right)$ pairs $1 \leq i<j \leq k,\left|d\left(U_{i}, U_{j}\right)-d\left(V_{\pi(i)}, V_{\pi(j)}\right)\right| \leq \epsilon$.

They prove that for some $f(k)=\Theta\left(\frac{\log ^{1 / 3} k}{k^{1 / 3}}\right)$ and infinitely many $k$, and for every $n>n(k)$, there is a graph on $n$ vertices with two $f$-regular partitions which are not $1 / 4$-isomorphic. On the other hand, they show that if $f(k) \leq \min \left(1 / k^{2}, \epsilon / 2\right)$, then any two equitable partitions of $G$ into $k$ parts which are each $f$-regular must be $\epsilon$-isomorphic.

\section{- Multicolor and directed regularity and removal lemmas}

The proof of Szemerédi's regularity lemma has been extended to give multicolor (see [27]) and directed (see [7]) extensions. These imply multicolor and directed generalizations of the graph removal lemma. As discussed in [18, the new proof of the graph removal lemma with a logarithmic tower height extends with a similar bound to these versions as well. Axenovich and Martin [11] recently extended the strong regularity lemma in a similar fashion to give multicolor and directed versions, in order to establish extensions of the induced graph removal lemma. Our proof of the induced graph removal lemma with a tower-type bound similarly extends to give multicolor and directed versions.

\section{- On proofs of regularity lemmas}

As noted by Gowers, the constructions in [22] not only show that the bound in Szemerédi's regularity lemma is necessarily large, but that, in some sense, the proof is necessary. Any proof must follow a long sequence of successively finer partitions, each exponentially larger than the previous one. While this notion is hard to make precise, it should be clear to anyone who has studied the proof of the regularity lemma and the lower bound construction of Gowers. Theorem 1.1 adds further weight to this conviction. Furthermore, the proof of Theorem 1.2 shows that any proof of the strong regularity lemma requires a long sequence of partitions, each of tower-type larger than the previous partition. That is, the iterated use of Szemerédi's regularity lemma is required in any proof of the strong regularity lemma.

Acknowledgment. We would like to thank Noga Alon for helpful comments.

Note added. After this paper was written we learned that a variant of Theorem 1.2 was also proved, independently and simultaneously, by Kalyanasundaram and Shapira. In the situation of Corollary 1.2. their theorem gives a lower bound of wowzer-type in $\sqrt{\log \epsilon^{-1}}$ for the strong regularity lemma.

\section{References}

[1] M. Ajtai and E. Szemerédi, Sets of lattice points that form no squares, Stud. Sci. Math. Hungar. 9 (1974), 9-11. 
[2] N. Alon, Testing subgraphs in large graphs, Random Structures Algorithms 21 (2002), 359-370.

[3] N. Alon, R. A. Duke, H. Lefmann, V. Rödl, and R. Yuster, The algorithmic aspects of the regularity lemma, J. Algorithms 16 (1994), 80-109.

[4] N. Alon, W. Fernandez de la Vega, R. Kannan, and M. Karpinski, Random sampling and approximation of MAX-CSPs, J. Comput. System Sci. 67 (2003), 212-243.

[5] N. Alon, E. Fischer, M. Krivelevich, and M. Szegedy, Efficient testing of large graphs, Combinatorica 20 (2000), 451-476.

[6] N. Alon, E. Fischer, and I. Newman, Efficient testing of bipartite graphs for forbidden induced subgraphs, SIAM J. Comput. 37 (2007), 959-976.

[7] N. Alon and A. Shapira, Testing Subgraphs in Directed Graphs, J. Comput. System Sci. 69 (2004), $354-382$.

[8] N. Alon and A. Shapira, A characterization of easily testable induced subgraphs. Combin. Probab. Comput. 15 (2006), 791-805.

[9] N. Alon, A. Shapira, and U. Stav, Can a graph have distinct regular partitions? SIAM J. Discrete Math. 23 (2008/09), 278-287.

[10] N. Alon and J. H. Spencer, The probabilistic method, 3rd ed., Wiley, 2008.

[11] M. Axenovich and R. Martin, A version of Szemerédi's regularity lemma for multicolored graphs and directed graphs that is suitable for induced graphs, arXiv: 1106.2871.

[12] N. Bansal and R. Williams, Regularity lemmas and combinatorial algorithms, Proceedings of the 50th IEEE FOCS (2009), 745-754.

[13] B. Bollobás, The work of William Timothy Gowers, Proceedings of the International Congress of Mathematicians, Vol. I (Berlin, 1998). Doc. Math., Extra Vol. I, 1998, 109-118 (electronic).

[14] F. R. K. Chung, R. L. Graham, R. M. Wilson, Quasi-random graphs, Combinatorica 9 (1989), $345-362$.

[15] R. A. Duke, H. Lefmann, and V. Rödl, A fast approximation algorithm for computing the frequencies of subgraphs in a given graph. SIAM J. Comput. 24 (1995), 598-620.

[16] N. Eaton, Ramsey numbers for sparse graphs, Discrete Math. 185 (1998), 63-75.

[17] P. Erdős, P. Frankl, and V. Rödl, The asymptotic number of graphs not containing a fixed subgraph and a problem for hypergraphs having no exponent, Graphs Combin. 2 (1986), 113-121.

[18] J. Fox, A new proof of the graph removal lemma, Ann. of Math. 174 (2011) 561-579. 
[19] A. Frieze and R. Kannan, The regularity lemma and approximation schemes for dense problems, Proceedings of the 37th IEEE FOCS (1996), 12-20.

[20] A. Frieze and R. Kannan, Quick approximation to matrices and applications, Combinatorica 19 (1999), 175-220.

[21] O. Goldreich, S. Goldwasser, and D. Ron, Property testing and its applications to learning and approximation, J. ACM 45 (1998), 653-750.

[22] W. T. Gowers, Lower bounds of tower type for Szemerédi's uniformity lemma, Geom. Funct. Anal. 7 (1997), 322-337.

[23] R. L. Graham, B. L. Rothschild, and J. H. Spencer, Ramsey theory, 2nd edition, John Wiley \& Sons (1980).

[24] P. E. Haxell, Partitioning complete bipartite graphs by monochromatic cycles, J. Combin. Theory Ser. B 69, (1997), 210-218.

[25] W. Hoeffding, Probability inequalities for sums of bounded random variables, J. Amer. Statist. Assoc. 58 (1963), 13-30.

[26] Y. Kohayakawa and V. Rödl, Szemerédi's regularity lemma and quasi-randomness, in Recent advances in algorithms and combinatorics, CMS Books Math./Ouvrages Math. SMC, 11, Springer, New York, 2003, 289-351.

[27] J. Komlós and M. Simonovits, Szemerédi's regularity lemma and its applications in graph theory, in Combinatorics, Paul Erdős is eighty, Vol. 2 (Keszthely, 1993), Bolyai Soc. Math. Stud. 2, János Bolyai Math. Soc., Budapest, 1996, 295-352.

[28] M. Krivelevich and B. Sudakov, Pseudo-random graphs, in More sets, graphs and numbers, Bolyai Soc. Math. Stud. 15, Springer, Berlin, 2006, 199-262.

[29] L. Lovász and B. Szegedy, Szemerédi's lemma for the analyst, Geom. Funct. Anal. 17 (2007), $252-270$.

[30] M. Malliaris and S. Shelah, Regularity lemmas for stable graphs, arXiv:1102.3904.

[31] Y. Peng, V. Rödl, and A. Ruciński, Holes in graphs, Electron. J. Combin. 9 (2002), Research Paper 1, 18 pp. (electronic).

[32] V. Rödl and M. Schacht, Regular partitions of hypergraphs: regularity lemmas, Combin. Probab. Comput. 16 (2007), 833-885.

[33] V. Rödl and M. Schacht, Regularity lemmas for graphs, in Fete of Combinatorics and Computer Science, Bolyai Soc. Math. Stud. 20, 2010, 287-325.

[34] K. F. Roth, On certain sets of integers, J. London Math. Soc. 28 (1953), 104-109. 
[35] R. Rubinfield and M. Sudan, Robust characterization of polynomials with applications to program testing, SIAM J. Comput. 25 (1996), 252-271.

[36] I. Z. Ruzsa and E. Szemerédi, Triple systems with no six points carrying three triangles, in Combinatorics (Keszthely, 1976), Coll. Math. Soc. J. Bolyai 18, Volume II, 939-945.

[37] J. Solymosi, Note on a generalization of Roth's theorem, in Discrete and computational geometry, Algorithms Combin. Vol. 25, Ed. János Pach, Springer, 2003, 825-827.

[38] E. Szemerédi, On graphs containing no complete subgraph with 4 vertices, Mat. Lapok 23 (1972), $113-116$.

[39] E. Szemerédi, Integer sets containing no $k$ elements in arithmetic progression, Acta Arith. 27 (1975), 299-345.

[40] E. Szemerédi, Regular partitions of graphs, in Colloques Internationaux CNRS 260 - Problèmes Combinatoires et Théorie des Graphes, Orsay (1976), 399-401.

[41] T. Tao, Szemerédi's regularity lemma revisited, Contrib. Discrete Math. 1 (2006), 8-28.

[42] A. G. Thomason, Pseudorandom graphs, in Random graphs '85 (Poznań, 1985), North-Holland Math. Stud. Vol. 144, North-Holland, Amsterdam, 1987, 307-331. 